\title{
Trajectory Optimization for a Mission to Neptune and Triton
}

\author{
Thesis Report
}

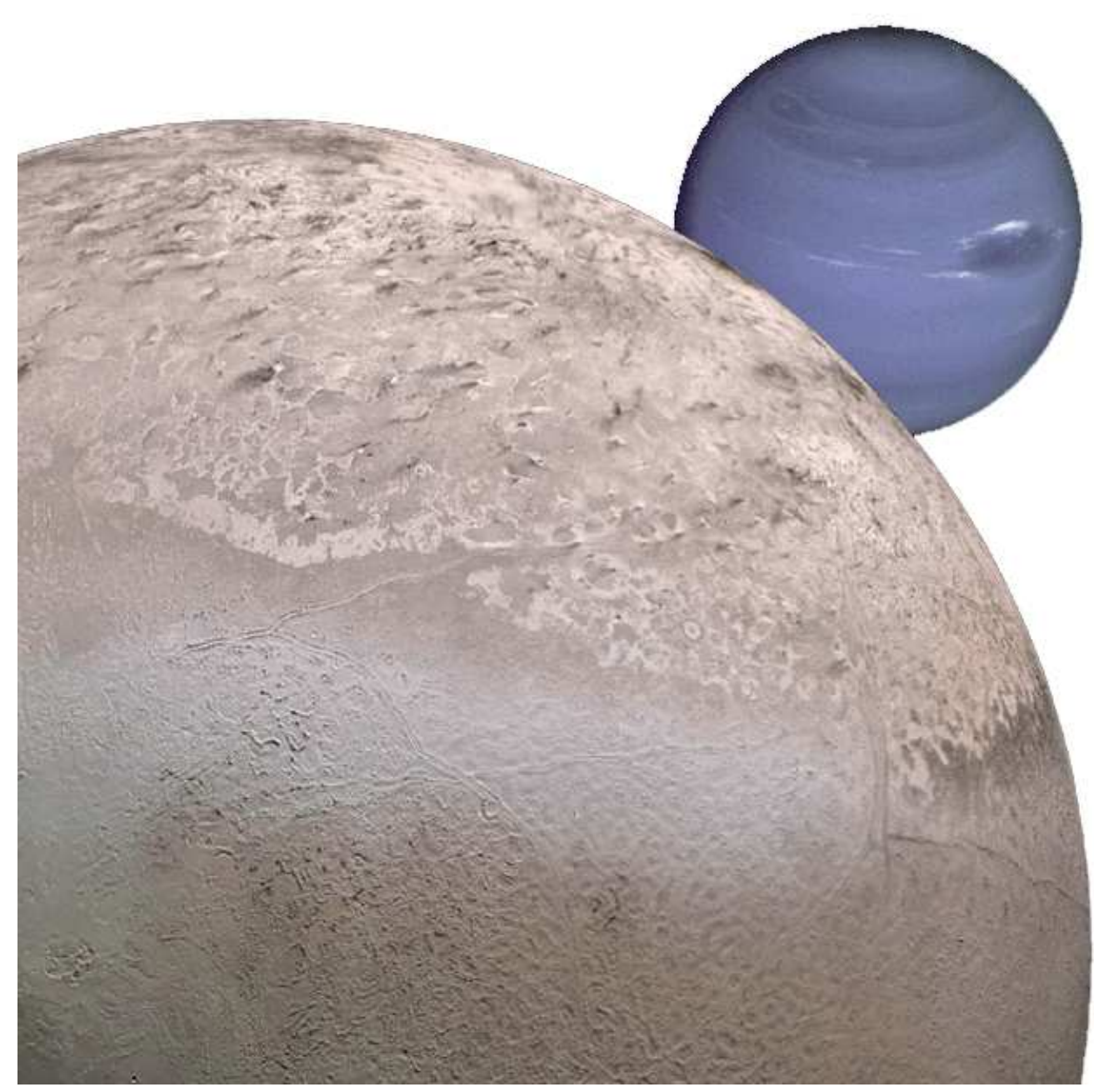

Jeroen Melman

18 January 2007 



\title{
Trajectory Optimization for a Mission to Neptune and Triton
}

\author{
Thesis Report
}

Jeroen Melman

18 January 2007

Astrodynamics \& Satellite Systems

Faculty of Aerospace Engineering

Delft University of Technology

Kluyverweg 1, 2629 HS Delft

The Netherlands 



\section{Preface}

In January 2006 I had just returned from my internship in Spain, and started on the final part of my studies in Aerospace Engineering at Delft University of Technology: my Master of Science thesis. During my internship I had already shown interest for a particular thesis project to be started up at the chair of Astrodynamics and Satellite Systems. This project comprises a mission to Neptune, with an emphasis on the trajectory, and was proposed by dr. Ortega from the European Space Agency [Ortega, 2005].

I was not the only one interested, also Ebrahim Safipour liked the idea of working on this project. This did not have to result in a competition of who could work on it, since it comprised more than enough work for multiple persons. After a thorough investigation of the work package, even the need was found for a third person, who was found in the person of Giovanni Orlando. The following work division was agreed upon: the launch and interplanetary trajectory would be done by me, capture at Neptune and an investigation of the Neptunian system by Ebrahim Safipour, and finally the release of a Triton orbiter and capture at Triton by Giovanni Orlando. I was the first to start on this project, which was one of the reasons to do the first part of this mission. The other two are planned to finish in the course of 2007.

This work would have been impossible to accomplish without the help of many people. First of all, the other MSc students on the ninth floor of the Aerospace Faculty building created a pleasant atmosphere to work in. In particular, I want to thank my thesis supervisor Ron Noomen, who always kept the door open for questions, and helped me to stay focused on the larger picture. Also I would like to thank dr. Ortega from ESA for starting up this challenging project. A special gratitude goes out to my girl friend, friends and family, for supporting me during the hard work.

Jeroen Melman 



\section{Contents}

Preface $\quad$ v

List of Tables $\quad$ xi

List of Figures $\quad$ xiii

Nomenclature $\quad$ xv

1 Introduction 1

2 The Neptunian System $\quad 3$

2.1 Neptune ............................. 3

2.2 Moons and Rings . . . . . . . . . . . . . . . . 6

2.2 .1 Triton ...................... 6

2.2 .2 Multiple Moons . . . . . . . . . . . . . . . . 8

2.2 .3 Rings $\ldots \ldots \ldots \ldots \ldots \ldots \ldots$

2.3 Science Objectives . . . . . . . . . . . . . . . . . . 9

2.4 Timeline .............................. 9

3 Principles of Astrodynamics 11

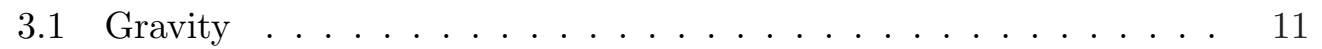

3.2 Keplerian Orbits . . . . . . . . . . . . . . . . . 12

3.2 .1 The Ellipse . . . . . . . . . . . . . . . . 14

3.2 .2 The Hyperbola . . . . . . . . . . . . . . . . . . 14

3.3 Third-Body Effects (Qualitative) . . . . . . . . . . . 16

3.4 Planetary Swing-By . . . . . . . . . . . . . . . 17 
4.1 Gravity Field Perturbations . . . . . . . . . . . . . . . . . 23

4.2 Atmospheric Drag . . . . . . . . . . . . . . . . 25

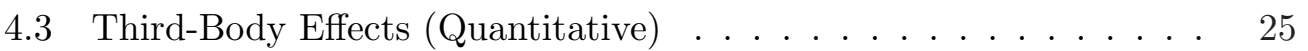

4.4 Radiation Pressure . . . . . . . . . . . . . . . . . . . 26

4.5 Electro-Magnetic Effects . . . . . . . . . . . . . . . . . . 28

4.6 Relativistic Effects . . . . . . . . . . . . . . . . 30

4.7 Implications on Trajectory . . . . . . . . . . . . . . . . . . 31

5 Propulsion 33

5.1 Chemical Propulsion . . . . . . . . . . . . . . . . . . . 33

5.1 .1 Basic Principles . . . . . . . . . . . . . . . . 33

5.1 .2 Methods . . . . . . . . . . . . . . . . . 34

5.1 .3 Practice ......................... 35

5.2 Electric Propulsion . . . . . . . . . . . . . . . . . . . 36

$5.2 .1 \quad$ Basic Principles . . . . . . . . . . . . . . . . . 36

5.2 .2 Methods . . . . . . . . . . . . . . . . 36

$5.2 .3 \quad$ Practice . . . . . . . . . . . . . . . 37

5.3 Launch Systems . . . . . . . . . . . . . . . . . . . . . . . 38

6 High-Thrust Trajectories 41

6.1 Patched Conics . . . . . . . . . . . . . . . . . . . . 41

6.1.1 Three-Dimensional Trajectories . . . . . . . . . . . 43

6.2 Lambert Targeting . . . . . . . . . . . . . . . . . . . . 44

6.3 Powered Gravity Assist _ . . . . . . . . . . . . . . . . . 48

6.4 The Aerogravity Assist . . . . . . . . . . . . . . . . . . 50

7 Low-Thrust Trajectories $\quad 55$

7.1 General Characteristics . . . . . . . . . . . . . 55

7.2 Shape and Dynamics . . . . . . . . . . . . . . . . 56

7.2 .1 Out-of-Plane Motion . . . . . . . . . . . . 58

7.3 Algorithm . . . . . . . . . . . . . . . . . . 60 
7.3 .1 Finding Intersections $\ldots \ldots \ldots \ldots$

7.4 Verification ...................... 63

8 Optimization $\quad 65$

8.1 Methods Overview . . . . . . . . . . . . . . . . 65

8.1.1 Calculus-based Methods . . . . . . . . . . . . . 65

8.1.2 Enumerative Methods . . . . . . . . . . . . . . 66

8.1.3 Random Methods . . . . . . . . . . . . . . . . 67

8.2 Evolution Programs _ . . . . . . . . . . . . . . . 67

8.3 Multi-Objective Optimization . . . . . . . . . . . . . 70

9 Galomusit $\quad 73$

9.1 History . . . . . . . . . . . . . . . . . . . 73

9.2 General Structure . . . . . . . . . . . . . . . . . . . . 74

9.3 Adaptations to Galomusit . . . . . . . . . . . . . . . . . . . 74

9.3.1 Generation of Individuals . . . . . . . . . . . . . 76

9.4 Setting the Evolution Program _... . . . . . . . . 76

9.5 Verification of Galomusit . . . . . . . . . . . . . . . . 79

$\begin{array}{ll}10 \text { Results } & 81\end{array}$

10.1 Settings and Boundary Conditions . . . . . . . . . . . . 81

10.2 Results for High-Thrust Trajectories . . . . . . . . . . . . 83

10.3 Results for Low-Thrust Trajectories . . . . . . . . . . . . . . . 92

$\begin{array}{ll}11 \text { Conclusion } & 99\end{array}$

12 Recommendations 101

$\begin{array}{ll}\text { Bibliography } & 103\end{array}$

A Derivations Petropoulos I 



\section{List of Tables}

2.1 Data on Neptune and Triton _............... . 4

3.1 Spheres of influence for selected planets and moons . . . . . . . 18

3.2 Limits on periapsis distance . . . . . . . . . . . . 20

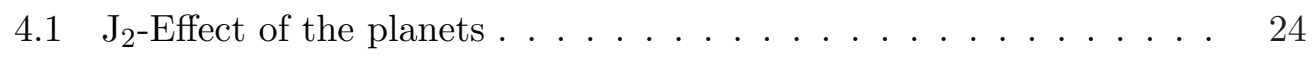

4.2 Third-body disturbances during Jupiter flyby . . . . . . . . . . 26

4.3 Third-body disturbances between Uranus and Neptune . . . . . . . 27

4.4 Received power density at the planets . . . . . . . . . . 27

4.5 Magnetic field as a disturbance . . . . . . . . . . . . . . 29

5.1 Available liquid rocket engines . . . . . . . . . . . 35

5.2 Primary electric propulsion in practice . . . . . . . . . 37

5.3 Cost of various launch vehicles . . . . . . . . . . . . 39

6.1 Height during an aerogravity assist . . . . . . . . . . 54

6.2 Acceleration during an aerogravity assist . . . . . . . . . 54

9.1 Evolution programs and population size . . . . . . . . 78

9.2 Verification using the old Galomusit . . . . . . . . . . . 79

9.3 Verification using Swing-By Calculator . . . . . . . . . 80

10.177 Different sequences to reach Neptune . . . . . . . . . 83

10.2 The best pure gravity assist trajectory in numbers, 2010-2030 . . . 85

10.3 The best aerogravity assist trajectory in numbers, 2010-2030 _ . . 87

10.4 The best pure gravity assist trajectory in numbers, 2013-2028 . . . 89

10.5 The best aerogravity assist trajectory in numbers, 2013-2028 . . . 90 
10.6 The shortest aerogravity assist trajectory in numbers . . . . . . . 91

10.7 Velocity input for capture calculations . . . . . . . . . . . . . 91 


\section{List of Figures}

2.1 Neptune and the Great Dark Spot . . . . . . . . . . . 5

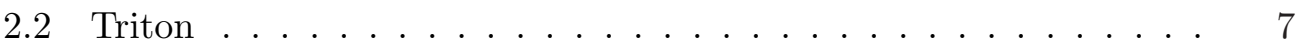

2.3 Cantaloupe terrain . . . . . . . . . . . . . . . 7

2.4 The rings of Neptune . . . . . . . . . . . . . . . . 8

3.1 Position vectors in the many-body problem . . . . . . . . . . . 12

3.2 The general conic section and a specific case: the ellipse . . . . . 13

3.3 Dependence of velocity on distance . . . . . . . . . . . 15

3.4 Relative positions of three bodies . . . . . . . . . . . . . . 16

3.5 The geometry of hyperbolic encounter trajectories _ . . . . . . 19

3.6 Vector diagram of velocities of gravity assist . . . . . . . . . . 19

3.7 Maximum heliocentric energy increase for flybys . . . . . . . . . 21

4.1 Definition of spherical coordinates . . . . . . . . . . . 24

5.1 Generic layout of a liquid rocket engine . . . . . . . . . . 35

$5.2 \quad$ SMART-1's electric engine . . . . . . . . . . . . . . . 37

5.3 Earth escape performance of various launch vehicles $\ldots \ldots$. . . . 39

5.4 Various launch vehicles in the early phases of launch . . . . . . . 40

6.1 Geometry of two-dimensional interplanetary trajectory . . . . . . . 43

6.2 Geometry of Lambert problem . . . . . . . . . . . . . . 45

6.3 Two solutions for an Earth-Mars transfer . . . . . . . . . . . . . 45

6.4 Plot of $T$ versus $x$ for selected values of $q$ and $m \ldots \ldots$

6.5 Geometry of a powered swing-by . . . . . . . . . . . . 49

6.6 Geometry for the determination of $\Delta V_{2} \ldots \ldots \ldots \ldots$ 
6.7 The forces on a spacecraft during an aerogravity assist . . . . . . 51

6.8 Geometry of an aerogravity assist . . . . . . . . . . . . . . 52

7.1 Optimized low-thrust interplanetary trajectory $\ldots \ldots \ldots 6$

7.2 Sample exponential sinusoids . . . . . . . . . . . . . . 57

7.3 Out-of-plane thrusting . . . . . . . . . . . . . . . . . 60

7.4 Exponential sinusoid intersections with Mars . . . . . . . . . 63

7.5 Miss angle as a function of $k_{2} \ldots \ldots \ldots \ldots$

7.6 Thrust acceleration profile of an exponential sinusoid . . . . . . . . 64

8.1 A multiple peak function . . . . . . . . . . . . . 66

8.2 The basic steps in Evolution Programs . . . . . . . . . . . . . 68

8.3 Weighted roulette wheel used for simple selection . . . . . . . . 69

9.1 Flow diagram of Galomusit _ . . . . . . . . . . . . . . 75

9.2 Fitness as a function of generation $\ldots \ldots \ldots \ldots$

10.1 Position of the planets in 2010 and $2030 \ldots \ldots$. . . . . . 82

10.2 Best sequences to Neptune, 2010-2030, pure gravity assists . . . . . 83

10.3 Best pure gravity assist trajectory . . . . . . . . . . . . 86

10.4 Detail of best pure gravity assist trajectory . . . . . . . . . 86

10.5 Best sequences to Neptune, 2010-2030, aerogravity assists . . . . . 87

10.6 Best sequences to Neptune, 2013-2028, pure gravity assists . . . . . 88

10.7 Best sequences to Neptune, 2013-2028, aerogravity assists . . . . . 89

10.8 Propellant mass fraction against the launch date, broad search . . 94

10.9 Final mass against time of flight, broad search _ . . . . . . . . 95

10.10Final mass against time of flight, refined search . . . . . . . . 96

10.11The best found low-thrust trajectory to Neptune . . . . . . . . . 96

10.12Thrust profile of exponential sinusoid . . . . . . . . . . . . 97 
Nomenclature

\section{Roman Symbols}

\begin{tabular}{ll}
$a$ & Acceleration \\
$a$ & Semi-Major Axis \\
$b$ & Semi-Minor Axis \\
$B$ & Impact Parameter \\
$B$ & Magnetic Field Strength \\
$c$ & Speed of Light: $2.99792458 \cdot 10^{8}$ \\
$C$ & Capacitance \\
$D$ & Drag \\
$e$ & Eccentricity \\
$\mathcal{E}$ & Total Energy \\
$f$ & Objective Function \\
$F$ & Force \\
$g$ & Gravitational Acceleration \\
$G$ & Gravitational Constant: $6.67259 \cdot 10^{-11}$ \\
$H$ & Angular Momentum \\
$H$ & Scaling Height \\
$i$ & Inclination \\
$I$ & Impulse \\
$J_{n}^{m}$ & Constant of the Gravitational Field \\
$k$ & Boltzmann's Constant: $1.380658 \cdot 10^{-23}$ \\
$k_{0}$ & Scale Parameter \\
$k_{1}$ & Dynamic Range Parameter \\
$k_{2}$ & Winding Parameter \\
$L$ & Lift \\
$\dot{m}$ & Fuel Mass Flow \\
$m$ & Mass \\
$p$ & Semi-Latus Rectum \\
$P$ & Power \\
$P_{n}$ & Legendre Function \\
$q$ & Electric Charge \\
$r$ & Radius \\
$R$ & Averaged Equatorial Body Radius \\
$R$ & Reflection Coefficient \\
$s$ & Semi-Perimeter \\
$S$ & Surface Area \\
$t$ & Time \\
$T$ & Revolution Period \\
& \\
\hline & \\
\hline & \\
\hline & \\
\hline & \\
\hline &
\end{tabular}

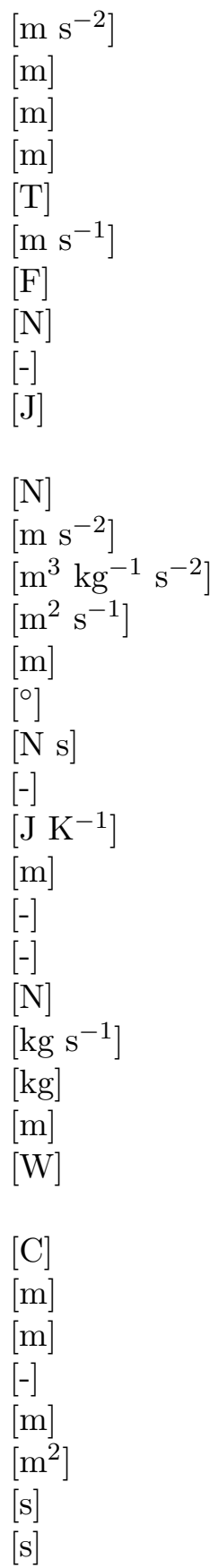


$T$

$u$

$U$

V

V

W

$\alpha$

$\beta$

$\gamma$

$\delta$

$\Delta$

$\zeta$

$\eta$

$\theta$

$\theta$

$\lambda$

$\lambda_{n}^{m}$

$\Lambda$

$\mu$

$\rho$

$\sigma$

$\phi$

$\varphi$

$\Phi$

$\omega$

$\Omega$

0

1

2

3

a

$a$

$a b$

alb

$a v g$

b

$c$

$c$

cap

$d$

e

esc

ext

E
Thrust

Reciprocal of the Radius $r$

Gravity Potential

Velocity

Voltage

Power Density

\section{Greek Symbols}

Deflection Angle

Hyperbolic Encounter Angle

Flight Path Angle

Geocentric Latitude

Change

Angle of Incidence

Thrust Efficiency

Polar Angle

True Anomaly

Geocentric Longitude

Constant of the Gravitational Field

Mass Ratio

Gravitational Parameter

Density

Standard Deviation

Phase Angle

Polar Angle

Magnetic Latitude

Argument of Perigee

Right Ascension of the Ascending Node

\section{Subscripts}

Closest to Earth in Hohmann Trajectory (HT)

At Radius of Influence of the Earth in HT

At Radius of Influence of Target in HT

Closest to Target Planet in Hohmann HT

Apoapsis

Atmospheric

Aerodynamic Bending

Albedo

Average

Burn-Out

Circular Parking Orbit

Circumferential

Capture

Disturbing Body

Exhaust

Escape

Extra

Earth
$[\mathrm{N}]$

$\left[\mathrm{m}^{-1}\right]$

$\left[\mathrm{m}^{2} \mathrm{~s}^{-2}\right]$

$\left[\mathrm{m} \mathrm{s}^{-1}\right]$

[V]

$\left[\mathrm{W} \mathrm{m}^{-2}\right.$ ] $\left[^{\circ}\right]$
$\left[^{\circ}\right]$
$\left[^{\circ}\right]$
$\left[^{\circ}\right]$
$[-]$
$\left[^{\circ}\right]$
$[-]$
$\left[^{\circ}\right]$
$\left[^{\circ}\right]$
$\left[^{\circ}\right]$
$\left[^{\circ}\right]$
$\left[^{\circ}\right]$
$\left[\mathrm{m}^{3} \mathrm{~s}^{-2}\right]$
$\left[\mathrm{kg} \mathrm{m}^{-3}\right]$

$\left.{ }^{\circ}\right]$

$\left[{ }^{\circ}\right]$

$\left.{ }^{\circ}\right]$

$\left[{ }^{\circ}\right]$

$\left[^{\circ}\right]$ 


$\begin{array}{ll}f & \text { Final } \\ i & \text { Initial } \\ i, j, k & \text { Identifiers for Different Bodies } \\ i t & \text { Interplanetary Trajectory } \\ l & \text { Lorentz } \\ m & \text { Main } \\ m & \text { Order of Legendre Function } \\ \text { max } & \text { Maximum } \\ \text { min } & \text { Minimum } \\ n & \text { Degree of Legendre Function } \\ N & \text { Neptune } \\ \text { out } & \text { Out-of-Plane } \\ p & \text { Periapsis } \\ p a y l & \text { Payload } \\ p l & \text { Planet } \\ p r & \text { Propellant } \\ r & \text { Radial } \\ s & \text { Satellite } \\ s & \text { Surface } \\ s d & \text { Satellite to Disturbing Body } \\ s p & \text { Specific } \\ S & \text { Sun } \\ S o I & \text { Sphere of Influence } \\ t & \text { Target Planet } \\ T & \text { Thrust } \\ \infty & \text { Infinity }\end{array}$

\section{Acronyms}

AGA AeroGravity Assist

AU Astronomical Unit

DP Dynamic Programming

E Earth

EP Electric Propulsion

ESA European Space Agency

EvP Evolution Programming

FIT Fitness Value

FY Fiscal Year

GA Genetic Algorithm

GA Gravity Assist

HET Hall Effect Thruster

HT Hohmann Trajectory

ILS International Launch Services

ISS International Space Station

J Jupiter

LEO Low Earth Orbit

M Mars

MMH Mono-Methyl Hydrazine

MON Mixed Oxides of Nitrogen

MSc Master of Science 


$\begin{array}{ll}\mathrm{N} & \text { Neptune } \\ \mathrm{N}_{2} \mathrm{H}_{4} & \text { Hydrazine } \\ \mathrm{N}_{2} \mathrm{O}_{4} & \text { Nitrogen Tetroxide } \\ \text { NASA } & \text { National Aeronautics and Space Administration } \\ \text { OIS } & \text { Orbital Insertion Stage } \\ \text { RTG } & \text { Radioisotope Thermoelectric Generator } \\ \mathrm{S} & \text { Saturn } \\ \text { SBC } & \text { Swing-By Calculator } \\ \text { SEP } & \text { Solar Electric Propulsion } \\ \text { SMART } & \text { Small Missions for Advanced } \\ & \quad \text { Research and Technology } \\ \text { STA } & \text { Space Trajectory Analysis } \\ \text { SRB } & \text { Solid Rocket Booster } \\ \text { TOF } & \text { Time Of Flight } \\ \text { V } & \text { Venus }\end{array}$




\section{Abstract}

In the outskirts of our solar system Neptune is located. Its characteristics bring up many questions, like the origin of its largest moon, Triton. A spacecraft near Neptune and Triton will provide the opportunity to answer these questions. The aim of this MSc thesis is to find an optimal trajectory towards Neptune. Two other MSc thesis researches will be performed for this mission: E. Safipour will detail the capture at Neptune and the investigation of the Neptunian system, and G. Orlando will look into the release of a Triton orbiter and capture at Triton.

The spacecraft can make use of gravity assists: a momentum exchange between the spacecraft and a particular planet, resulting in an increase in heliocentric velocity in the order of several kilometers per second. The planets can be used even more effectively by flying through their atmosphere, by which the performance can be enhanced. These passive velocity changes are generally spoken not sufficient to follow the desired trajectory, and therefore on-board propulsion is required. Two options are treated: the extensively used chemical propulsion, and the maturing low-thrust propulsion. The latter option introduces a certain risk because of its limited experience, but as an advantage it can give a higher velocity increase for the same amount of propellant. From a study on the perturbing forces, it follows that these forces are small enough to be neglected for first order computations. Therefore only the thrust and the gravity of the main body (be it the Sun or the swing-by planet) will be taken into account, which enables the use of particular analytical techniques for trajectory calculations.

The trajectories related to the low-thrust engines are analytically modeled using so-called exponential sinusoids, a very novel technique in this field. Because of its analytical nature it should be able to provide mission designers with a rapid, broad overview of the design space. However, several limitations of this technique were identified, when applying it to the problem at hand. The most important one is that the associated thrust profile does not conform well to realistic profiles. Therefore, no feasible low-thrust trajectory has been identified.

In order to find an optimal trajectory, obviously an optimization method is required. A genetic algorithm has been implemented, since it is able to cope with large search spaces. This method exploits the 'survival-of-the-fittest' principle. It is found to have a good performance for this particular problem. The optimization parameter is taken to be the required velocity change for this mission, including launch, powered gravity assists, and capture. A software tool is needed to perform the involved calculations. A tool named Galomusit was already developed at the Faculty of Aerospace Engineering, Delft, that could be applied to this problem. 
Nonetheless, numerous adaptations were made. The most important one resulted in a decrease of the computation time of about two orders of magnitude.

Using this software tool, two best high-thrust trajectories (using chemical propulsion) have been identified. One implements pure gravity assists and the other one conducts aerogravity assists. The first one is a trajectory using five gravity assists: Venus, again Venus, Earth, Jupiter, and Saturn. Launch takes place in May 2012 and the spacecraft arrives at Neptune in December 2029. Assuming that a chemical capture is performed, the spacecraft mass will be $1226 \mathrm{~kg}$ when finally in orbit around Neptune. Using aerogravity assists, a trajectory via the atmosphere of Mars is identified as the best. Launch takes place in January 2014 and the spacecraft arrives at Neptune in June 2024. Assuming an aerocapture at Neptune, the final spacecraft mass will be $2364 \mathrm{~kg}$ (without aerocapture system). This is an approximate figure, since no detailed research has been performed to find the exact implications of an atmospheric flight on the spacecraft's mass.

The shorter flight time and higher mass for the optimal aerogravity assist trajectory might suggest that this trajectory is the best. However, this technique has not been applied in practice before, while a pure gravity assist has been used extensively. For the time being, no definitive choice can be made between these two trajectories. Therefore both options will be presented to the person performing the capture research. It can be concluded that well-performing trajectories to Neptune have been found, on which the continuation of the mission design, the trajectory in particular, can build further. 
Chapter 1

\section{Introduction}

From the beginning of its existence, mankind has been a curious race. Every time a discovery is made, it brings up new questions, for which we want to find an answer. It brought us to exploring the great seas and discovering new continents. Since the last century it is possible to go further than our own Earth. Many satellites and astronauts have gone beyond the confines of Earth's atmosphere to explore outer space. Missions have gone to multiple planets to learn more about the formation of our solar system, their habitability etc. However, Neptune has never been studied up close for a considerable period of time. A future mission to Neptune and its largest moon Triton is expected to give us more insight in planetary phenomena and answer numerous scientific questions. Such a mission poses many challenges, the trajectory towards the planet being one of them. Current technologies and techniques have to be used to their limits and developed further to be able to bring a spacecraft that far from the Sun.

The aim of this MSc thesis is to explore possibilities for a mission of the European Space Agency (ESA) to Neptune, the trajectory towards Neptune in particular. To this end multiple areas in engineering, physics and mathematics have been studied during the preparatory literature survey [Melman, 2006]. This work formed the basis for finding an optimal trajectory to Neptune. Two options exist for the propulsion on board the spacecraft: chemical or low-thrust engines. The first option has been studied and used extensively in the past few decades, while the second one has gained little experience, but has very promising characteristics, like a very high efficiency. The trajectories related to the low-thrust engines are modeled using so-called exponential sinusoids, a very novel technique in this field. Also so-called (aero)gravity assists will form part of the trajectory to Neptune, which can significantly enhance its performance by exploiting the gravity and atmospheres of the planets of our solar system.

The performance is measured by the amount of energy it requires [Ortega, 2005], thereby maximizing the payload mass at Neptune. The required energy is best represented by the required velocity change for this mission. The latest year of arrival was originally set at 2020 [Ortega, 2005]. This requirement turned out to be too strict (see sections 2.4 and 10.1), and the arrival is now required to be before 2030. Minimizing the time of flight also forms an objective in itself. Therefore multiple options need to be found in terms of different times of flight, but also different arrival conditions. In that way the proceedings of this project, being the continuation of this mission in the sphere of influence of Neptune, can 
be further dealt with by other students. To conclude, the objective of this thesis is to find an optimal trajectory to Neptune in terms of energy requirements, and thereby making a distinction between chemical and low-thrust engines. The means to obtain the primary objective, form in fact a secondary objective in itself: the further development of space mission analysis tools, which were already developed at the chair of Astrodynamics \& Satellite Systems, Delft University of Technology.

Chapter 2 will describe Neptune and its surroundings, from which the scientific objectives will follow. Chapter 3 deals with the principles of astrodynamics, constituting the basics required for this thesis work. Various perturbations of the spacecraft trajectory are discussed in chapter 4 . From this discussion the important conclusion can be drawn that they can be neglected for first order computations. The subject of chapter 5 is propulsion, which is obviously required to set course for Neptune. Both the high-thrust and the low-thrust techniques will be explained here. Using the findings from the previous 3 chapters, the core of this thesis work will be discussed in chapters 6 and 7, regarding the high-thrust and the low-thrust trajectories, respectively. These trajectories have to be optimized, so the method of optimization is treated in chapter 8. One cannot obtain results in this field of work without a computer program, so a tool was developed which is described in chapter 9 . The found results will be elaborately discussed in chapter 10. Naturally, a thesis report cannot go without a conclusion for which chapter 11 can be consulted. Finally, chapter 12 gives recommendations for future work in this area. 
Chapter 2

\section{The Neptunian System}

In this chapter the destination of the interplanetary mission at hand is described: Neptune and its surroundings. Naturally, Neptune is discussed first in section 2.1, after which its surroundings are dealt with (section 2.2). Finally the science objectives as they follow from the discussion of the Neptunian system are stated in section 2.3. The information in this chapter is from [Carroll and Ostlie, 1996], unless stated otherwise.

\section{$2.1 \quad$ Neptune}

In the outskirts of our solar system Neptune is located, one of the giant gas planets, also known as the Jovian worlds. Together with the other Jovian worlds, Jupiter, Saturn and Uranus, $99.5 \%$ of the entire mass of the planetary system is contained. Consequently, if we want to understand the development and evolution of our solar system, it is vital that we understand these distant worlds.

Neptune was discovered the last of the four, since it is furthest away, on September 23, 1846, by Johan Gottfried Galle, following the mathematical based predictions by John Couch Adams and Urbain Leverrier. These scientists came independently to almost the same results by considering the gravitational perturbations affecting the orbit of Uranus. Since the discovery of Neptune, the efforts of Earthbased astronomers have provided important information about Neptune and the other Jovian planets.

However, much of the data now available have come from space missions. First the Pioneers 10 and 11 flew by Jupiter and Saturn in the seventies. Later, Voyager 1 and 2 embarked on their spectacular "Grand Tour". Both Voyagers, launched from Earth in 1977, visited Jupiter (1979) and Saturn (1980,1981), while only Voyager 2 continued on to Uranus (1986) and Neptune (1989). The Pioneers and Voyagers all performed flybys: the planets could only be observed for a limited amount of time, since the spacecraft were not captured by one of the planets. Later missions have succeeded in this more difficult goal: to make extended observations of the destination planet. With respect to the gas giants two orbiter missions have been put into space: firstly the Galileo mission to Jupiter (first encounter in 1995), and secondly the Cassini-Huygens mission to Saturn and its largest moon Titan (first encounter in 2004). 


\begin{tabular}{|l|c|c|}
\hline & Neptune & Triton \\
\hline Radius $(\mathrm{km})$ & 24764 & 1303.4 \\
Mass $(\mathrm{kg})$ & $1.024 \cdot 10^{26}$ & $2.147 \cdot 10^{22}$ \\
Density $\left(\mathrm{kg} / \mathrm{m}^{3}\right)$ & $1.70 \cdot 10^{3}$ & $2.07 \cdot 10^{3}$ \\
Distance to Sun/Neptune $(\mathrm{km})$ & $4.497 \cdot 10^{9}$ & $3.548 \cdot 10^{5}$ \\
Orbital Period (years/days) & 165 & 5.88 \\
Inclination $\left(^{\circ}\right)$ & 1.774 & 157.34 \\
Eccentricity (-) & 0.0097 & 0.0000 \\
Rotation Period (days) & 0.768 & 5.88 \\
Surface Temperature $(\mathrm{K})$ & 53 & 37 \\
\hline
\end{tabular}

Table 2.1 Data on Neptune and Triton [Carroll and Ostlie, 1996; NASA/JPL, 2006c].

The Earth-based observations and the space missions have come up with some interesting results, which will now be discussed. To start off with the orbital data: it circles the Sun at a distance of about 30 AU. Since the eccentricity is a mere 0.0097 (only Venus has a smaller eccentricity) it can indeed be called circling by approximation. It takes 165 years to make a full orbit, which is inclined to the ecliptic by $1.774^{\circ}$, whereas the equator is tilted with respect to the orbit by $28.8^{\circ}$. The outer gas giant weighs more than 17 Earth masses with 3.88 times the Earth radius, resulting in an average density of $1.70 \cdot 10^{3} \mathrm{~kg} / \mathrm{m}^{3}$. These and more data on Neptune are given in table 2.1, together with data on Triton.

As a class the Jovian worlds differ significantly from the terrestrials (Mercury, Venus, Earth and Mars), since those consist primarily of rocky substances while the Jovians are gaseous, combined with liquids and ice. Then again the Jovian worlds can be subdivided. Jupiter and Saturn have average compositions that are quite similar to the Sun's with much hydrogen and helium, while the smaller and more distant Uranus and Neptune have higher proportions of heavier elements. The cores (containing most of the heavier elements) of Neptune and Uranus constitute $70 \%$ to $90 \%$ of their masses, whereas in the case of Jupiter and Saturn it is only $5 \%$ and $14 \%$ respectively. Clearly, Uranus and Neptune are not simply small versions of their larger siblings. And because each of the Jovians is capable of retaining all of the lighter elements in its atmosphere, this composition difference suggests differences in their formation. Thus, by making a detailed comparison of the internal structures and compositions, valuable insight into the processes that formed the solar system and, perhaps, other planetary systems can be obtained.

This mass distribution of the gas giants was determined by, amongst others, observing the motions of moons, rings and spacecraft. By comparing the actual motion of a satellite with what would be expected if the planet were a perfect sphere, it becomes possible to map the mass distribution of the interior. Probably the most evident feature of the non-sphericity is the oblateness of a planet, which describes how flattened it is. The amount of oblateness depends on the speed of rotation and the rigidity of the interior. Since the gas giants rotate faster than the Earth and are less rigid, the oblateness is higher. With oblateness defined as $\frac{R_{e}-R_{p}}{R_{e}}$, with $R_{e}$ the equatorial radius and $R_{p}$ the polar radius, it follows that Neptune has an oblateness of 0.022 , which is more than six times the Earth oblateness of 0.0034 . This oblateness will probably have a non-negligible effect on the orbit of the spacecraft to be designed. 


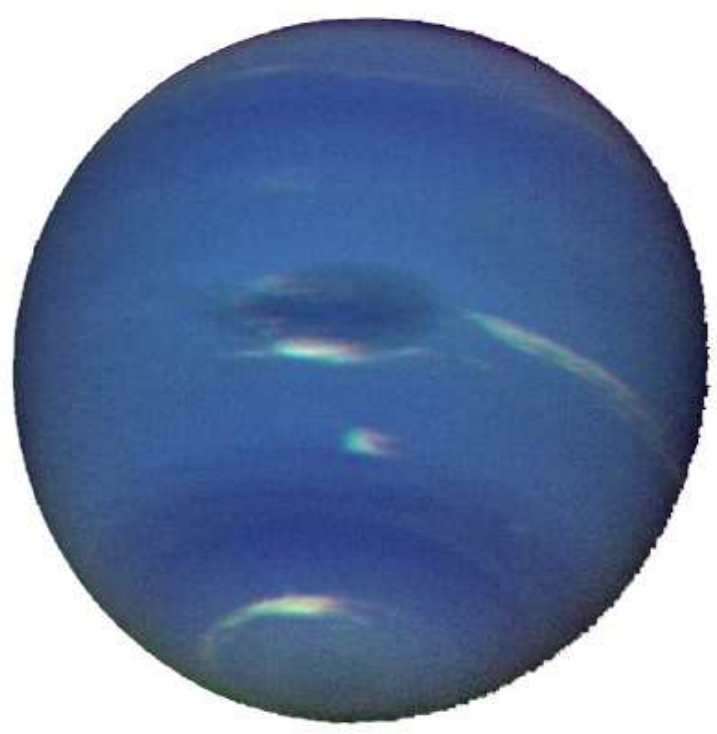

Figure 2.1 Neptune with the Great Dark Spot in the middle [NASA/JPL, 2006b].

In their upper atmospheres, the deep blue-green cloud tops of Uranus and Neptune (see figure 2.1) owe their beauty primarily to the constituents of the upper atmosphere. As sunlight passes through the atmosphere the blue wavelengths are scattered most efficiently by the molecules. In addition, the presence of methane in the atmosphere tends to absorb the red light. Another feature of its atmosphere is the Great Dark Spot (see figure 2.1), spotted by Voyager 2, with the size of Eurasia $[N A S A / J P L, 2005 b]$. It is not like the famous Great Red Spot of Jupiter, which has been observed for more than three centuries. The atmosphere of Neptune is very dynamic, not only on a small scale but also on a large scale, because the Great Dark Spot was not present when the Hubble Space Telescope pointed towards Neptune in 1994. It is possible that it was not a permanent cyclonic storm like the Great Red Spot, but an atmospheric hole like the ozone hole in the Earth atmosphere. Nevertheless there are very strong winds around this spot, up to $2,000 \mathrm{~km}$ an hour; the fastest winds in our solar system! [NASA/JPL, 2005b] Still many things remain unclear about this very dynamic atmosphere of Neptune.

The molten iron-nickel core of the Earth is the source of its magnetic field. In the Jovian planets this is not possible due to a deficit of iron and nickel. But due to the high pressures and temperatures in the core, hydrogen becomes liquid and metal-like, so it can fulfill the role of iron and nickel. Due to the rotation of this hydrogen a magnetic field is generated. In the case of Neptune the magnetic axis is tilted $47^{\circ}$ with respect to the rotation axis, and is offset more than half of its radius from its center. Due to this large offset the magnetic field is highly irregular when compared to the one on Earth. It is approximately ten times stronger on the southern hemisphere than on the northern hemisphere, with the strength of the Earth magnetic field lying in between. The exact cause of this exotic magnetic field is still not fully understood . 


\subsection{Moons and Rings}

The Neptunian system does not consist of Neptune alone. It has multiple moons and rings as well. Triton is the largest moon and will be discussed first in section 2.2.1. Then the other moons follow in section 2.2.2, and the rings close the discussion of the Neptunian system in section 2.2.3.

\subsubsection{Triton}

Seventeen days after the discovery of Neptune, its largest moon was discovered by William Lassell. It took 34 years after its discovery before its current name Triton was suggested. At a distance of $354,800 \mathrm{~km}$ it circles around Neptune in 5.877 days. It has a mean diameter of $2706.8 \mathrm{~km}$ and a mass of $2.147 \cdot 10^{22} \mathrm{~kg}$, resulting in an average density of $2.07 \mathrm{~g} / \mathrm{cm}^{3}$, almost the same as Neptune $[N A S A / J P L$, $2006 c$ ]. These and more data on Triton are given in table 2.1, together with data on Neptune. Triton is unique among the other large moons of our solar system, since it has a retrograde orbit. This implies that it could not be formed out of the same region as the solar nebula of the planets they orbit, but must be captured from elsewhere. Its similarity in size and composition to Pluto, as well as to Pluto's eccentric Neptune-crossing orbit, provides further hints to Triton's possible origin as a Pluto-like planetary body. Perhaps it finds its origin in the Kuiper Belt, a disk-shaped region of the solar system, extending from Neptune's orbit to about $50 \mathrm{AU}$, containing many small bodies.

Due to its retrograde motion the tidal interactions with its accompanying planet have different effects than in the case of the Earth-Moon system. In the latter case the tidal bulge caused by the tidal forces in combination with the rotation period of the Earth being shorter than the orbital period of the Moon, is slowing down the spinning of the Earth, while accelerating the Moon. Therefore the Moon will move away from the Earth. In the case of Triton the retrograde orbit will cause it to decelerate, and therefore it will spiral towards Neptune. Apparently it will take billions of years before any catastrophic interaction occurs. The tidal effect on the rotation of Triton itself has already come to an end: Triton is tidally locked with Neptune; just as our Moon always keeps the same face towards us. Another unique feature of the Triton orbit, arising from tidal effects on such a large moon so close to its primary, is that it is for all practical purposes a perfect circle with an eccentricity of zero to sixteen decimal places.

With a surface temperature of only $37 \mathrm{~K}$ it is also the coldest world in our solar system. The southern pole is covered with a pinkish frost that is composed almost entirely of nitrogen (see figure 2.2). Along with the nitrogen frost, other surface ices include methane, carbon monoxide (also called dry ice), and carbon dioxide. Also present are very large "frozen lakes" of water-ice that show very little cratering, indicative of a relatively young age. The water-ice may have erupted from ice volcanos. Due to the ice Triton is very bright, reflecting 60 to $95 \%$ of the sunlight that reaches it, while the Moon reflects only $11 \%$. Under the much different circumstances the surface ice can be quite different from the ice as we know it. For example large vaults can be formed, in combination with surface depressions, resulting in a remarkable surface (see figure 2.3). It is called "cantaloupe terrain", because it has the same appearance as this type of melon. 
The origin of these features is unknown, but there are a number of hypotheses, like viscous ice eruptions.

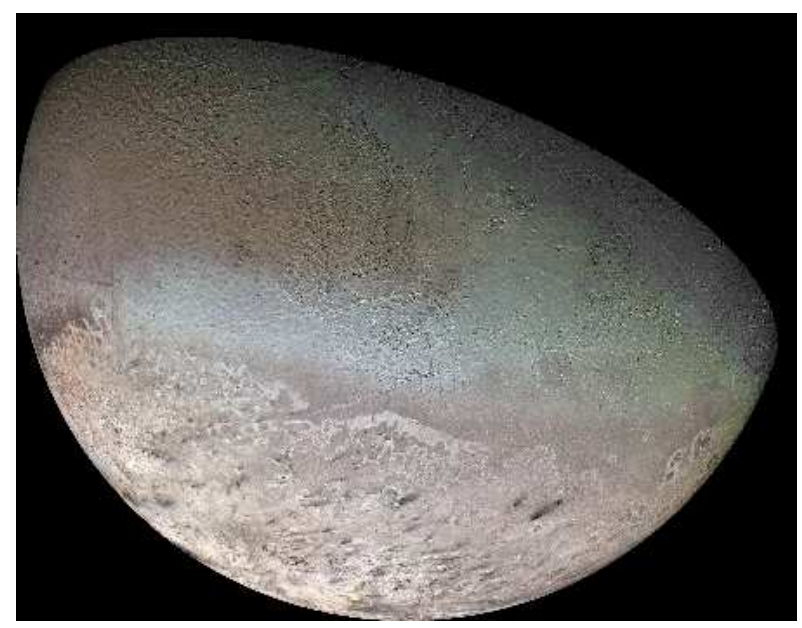

Figure 2.2 Triton with its pinkish southern pole [NASA/JPL, 2006b].

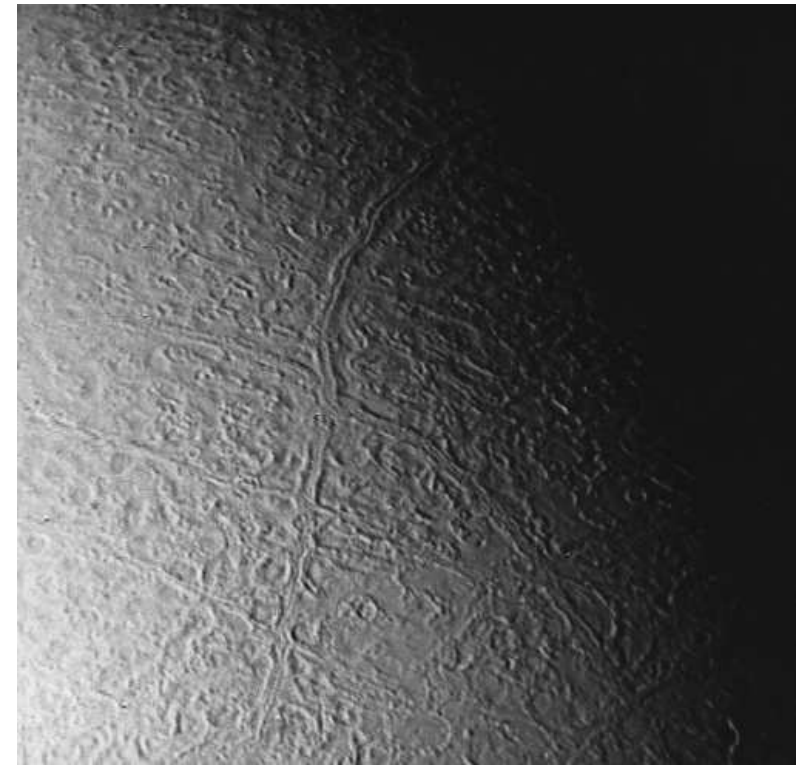

Figure 2.3 Cantaloupe terrain [NASA/JPL, 2006b].

The atmosphere of Triton is predominantly composed of nitrogen, like the atmosphere on Earth. However, the Triton atmosphere is extremely thin, with a pressure of only 1.6 $\mathrm{Pa}$ (compare to the Earth's atmospheric pressure at sea level of $1.01 \cdot 10^{5} \mathrm{~Pa}$ ). Much of the atmosphere may be a consequence of geyser-like jets of nitrogen gas erupting from the interior of the moon. These jets force plumes 8 $\mathrm{km}$ up into the tenuous atmosphere. These plumes may simply be gas rising from a warm source inside the planet, but how they are initiated remains unclear.

As always the following question arises: is life possible? The atmosphere is mainly composed of nitrogen, like on Earth, and there are traces of methane, which is normally associated with life; methane is then a by-product. But as already mentioned it is extremely cold on Triton and the atmosphere is almost vacuum. Therefore it is not suitable to any known life form. However, due to the geological activity and the possible internal heating, it is possible that below 
the surface of Triton there is a layer of liquid water, supported by antifreezers like ammonia. This subsurface ocean could sustain primitive forms of life.

\subsubsection{Multiple Moons}

Triton is not the only moon of Neptune. Twelve other moons exist in orbit around Neptune, but none of them are massive enough to be spheroidal, like Triton. The second known natural satellite is the irregularly shaped moon Nereid, having one of the most eccentric orbits of any moon in the solar system, i.e. $0.7512[N A S A / J P L$, $2006 c$ ]. In 1989 Voyager 2 discovered six new Neptunian moons: Proteus, Naiad, Thalassa, Despina, Galatea and Larissa. If Proteus would have been any larger, its own gravity would have pulled it into a spherical shape. In 2002 and 2003 five more moons were discovered. All but one remain unnamed, Psamathe being the named one. The fact that these moons were discovered just a few years ago, suggests that there might be more moons orbiting Neptune.

\subsubsection{Rings}

The most well-known set of planetary rings is that of Saturn, but also Neptune has rings. They are much less apparent because of their smaller dimensions, but most important because they contain dust instead of water-ice, the latter being much more reflective. They are so faint that they can only be seen if Neptune is masked (see figure 2.4). Partly because they are so faint there are still many features to be explained: the peculiar "clumpy" structure, the incompleteness of the rings (dividing the rings up into several arcs) and their instability.

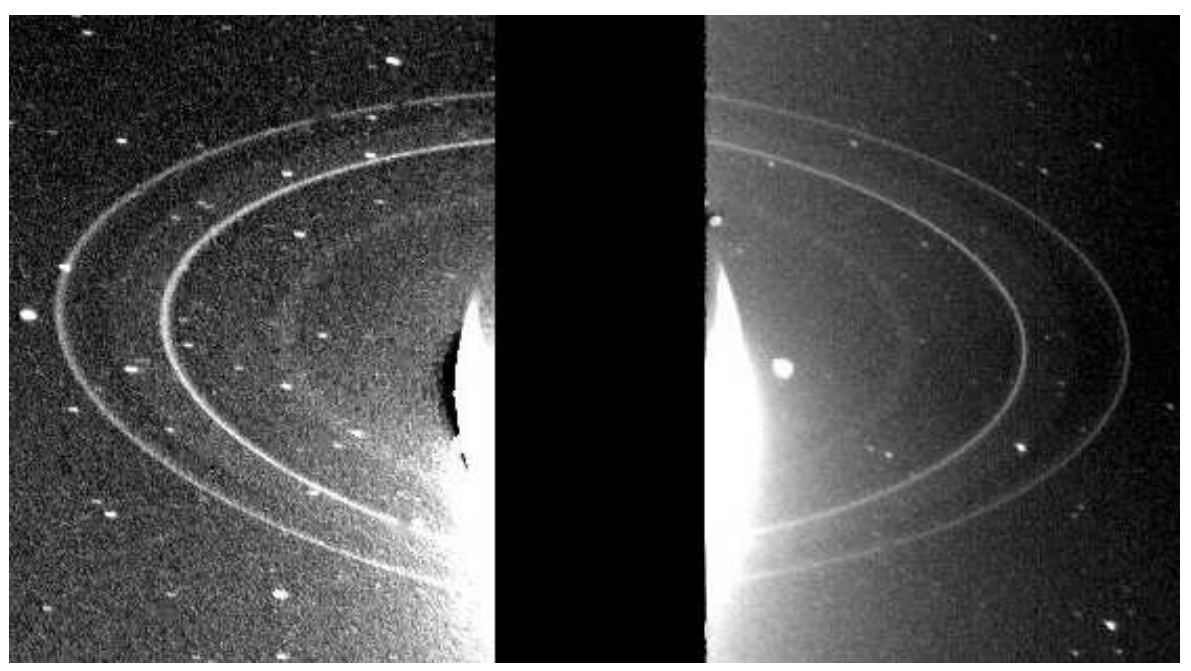

Figure 2.4 The rings of Neptune, with Neptune masked [NASA/JPL, 2006b]. 


\subsection{Science Objectives}

Although the Voyager has already flown by Neptune, this voyage was only a starter in the voracious appetites of the science community. Many aspects of the Neptunian system still remain unclear, as turned out from the discussion in this chapter. In this section a non-exhaustive overview is given of the science objectives in the form of questions.

- What is the atmospheric structure and chemistry of Neptune? What are the explanations for its already observed dynamic behavior?

- What is the structure and behavior of its magnetic field? Why does it so notably differ from the magnetic field of the Earth?

- What are the physical properties of Triton, both of the surface and of the interior? In particular, what are the causes for the cantaloupe terrain and gas plumes?

- Is Triton a captured Kuiper object? What can it tell us about the formation and evolution of the solar system?

- What are the dynamics of the rings and other moons, with possible new moons to be discovered?

- And the most exotic question of them all: does life exist on Triton, or did it in the past?

A spacecraft near Neptune and Triton will provide the opportunity to answer most of these questions.

\subsection{Timeline}

An orbiter mission to Neptune can take a considerable amount of time. Comparable missions to Jupiter (Galileo) and Saturn (Cassini) took 5 and 7 years, respectively, to arrive at their destination, and then orbited the planet for several years $[N A S A / J P L, 2005 a, 2006 a]$. To go to Neptune takes even a longer amount of time, since it is significantly further away (a semi-major axis of $30 \mathrm{AU}$ compared to one of 5 and $10 \mathrm{AU}$ for Jupiter and Saturn, respectively). As a baseline the results from [Noca and Bailey, 2004] are taken. They found flight times to Neptune in the order of 10 to 17 years. Shorter flight times (as low as 8 years) are shown to be possible, but at the cost of a significant increase in the required energy. This would result in a net delivered mass at Neptune (not inserted into Neptune's orbit) of less than $1000 \mathrm{~kg}$, while similar orbiter missions put a spacecraft in orbit weighing more than $2000 \mathrm{~kg}$ [Melman, 2006]. Also for the insertion more energy is required, since the faster trajectories imply higher velocities at Neptune [Noca and Bailey, 2004]. This discussion already indicates that the originally set requirement of an arrival before the year 2020 [Ortega, 2005] might not be realistic. This is further rationalized by the fact that the launch cannot take place tomorrow; some years should lie in between for developing, manufacturing and testing. In [Noca and Bailey, 2004] the launch years vary from 2012 to as late as 2018. In order to be more compliant with the originally set requirement, the first possible launch year is set to 2010 in this thesis. 
The original rationale behind the mentioned requirement (arrival before 2020) was partially for ESA to be able to compete or to cooperate with a mission performed by the National Aeronautics and Space Administration (NASA). In the 'Solar System Strategic Exploration Plans' by NASA, however, a Neptune Orbiter/Triton Explorer mission is foreseen not to be launched before 2030 [NASA, $2006 b]$. Therefore the originally required, relatively soon arrival is not necessary with respect to a possible NASA mission. Concluding, the requirement has been changed to reaching the Neptunian system before 2030. Effectively the time of flight is then limited to 20 years, but by changing the year of departure and the year of launch during the computations, also considerably shorter flight times will be obtained (see chapter 10). 
Chapter 3

\section{Principles of Astrodynamics}

In this chapter the basic theory behind the most important part of this graduation thesis will be discussed. Since the main focus is on the trajectory, the underlying principles of astrodynamics are this main point of attention. Gravity being the most important factor in all of this will be the starting point of this chapter.

\subsection{Gravity}

In nature there are four fundamental forces: gravitational, electromagnetic, strong nuclear and weak nuclear forces. Of those forces, gravity is the weakest on small scales, but the strongest on large scales. It does decrease with increasing scale (it is inversely proportional to the distance squared as evidenced by equation (3.1)), but the other three forces decrease much faster. And unlike the others it works on all matter and energy [Carroll and Ostlie, 1996]. Because of these reasons the interaction between objects in space is predominantly due to gravity. Astrodynamics or originally Celestial Mechanics uses this gravity to describe the movements of all objects that are in outer space: from comets to galaxies and from natural to artificial satellites. Gravity can be described by the following formula [Cornelisse et al., 1979]:

$$
\mathbf{F}=G \frac{m_{i} m_{j}}{\left|\mathbf{r}_{i j}\right|^{3}} \mathbf{r}_{i j}
$$

with $\mathbf{F}$ the gravity force exerted by body $j$ on body $i, G$ the universal gravitational constant, $m_{i}$ and $m_{j}$ the masses of the two attracting bodies and $\mathbf{r}_{i j}$ the distance vector from body $i$ to body $j$ (see figure 3.1). This formula does not take into account any relativistic effects, but for all practical purposes it is more than sufficient. To show this some attention will be devoted to this matter in section 4.6. In the following sections formula (3.1) will be used to determine the behavior of bodies in space. Not every derivation is described elaborately; that is not the intention of this document. For further in-depth information one is referred to a handbook, [Cornelisse et al., 1979] for example. 


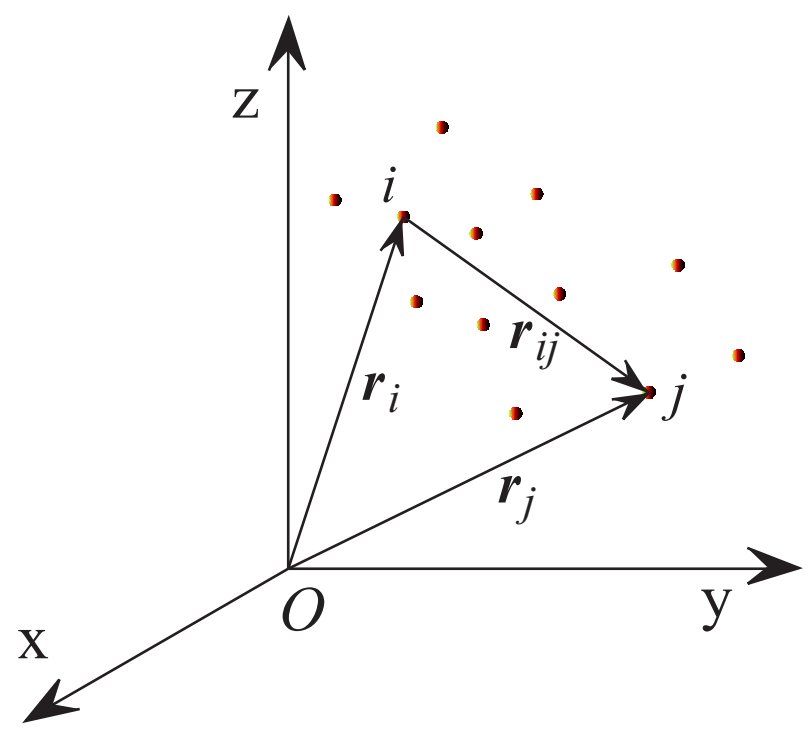

Figure 3.1 Position vectors in the many-body problem (adapted from [Cornelisse et al., 1979]).

As mentioned before all matter exerts a force on all other matter. This implies a complicated system of equations. For each body $i$ the following equation holds

$$
m_{i} \frac{d^{2} \mathbf{r}_{i}}{d t^{2}}=\sum_{j=1}^{n *} G \frac{m_{i} m_{j}}{r_{i j}^{3}} \mathbf{r}_{i j}
$$

with $n$ the total number of bodies. The index $*$ indicates that the summation excludes $j=i$. The definition of the distance vectors can be found in figure 3.1. Formula (3.2) can be further expanded into three scalar equations, rendering $3 n$ second-order coupled differential equations. For arbitrary values of $n$, it is impossible to solve this analytically. Therefore one has to fall back to numerical solutions. In some special cases this is not necessary: for some restricted three-body problems and all two-body problems there exist analytical solutions. The first set of problems, although very interesting, is not applicable to this thesis and therefore will be left aside. The second however, although a hypothetical case, will prove to be quite useful and will be discussed in the following section.

\subsection{Keplerian Orbits}

When considering a system of two bodies, the following equation can be derived from equation (3.1), describing the relative motion of body $i$ with respect to body $k$

$$
\ddot{\mathbf{r}}=-\frac{\mu}{r^{3}} \mathbf{r}
$$

with $\mu=G\left(m_{i}+m_{k}\right)$ and $\mathbf{r}$ the distance vector from body $k$ to body $i$. For a satellite of 1 ton moving around the Earth $m_{i} / m_{k}=1.7 \cdot 10^{-21}$, and orbiting around the Sun or Neptune renders an even smaller value, so one can also write as a very good approximation $\mu=G m_{k}$. Performing some clever operations on equation (3.3) results in the following conservation laws

$$
\frac{1}{2} V^{2}-\frac{\mu}{r}=\text { constant }=\mathcal{E}
$$




$$
\mathbf{r} \times \mathbf{V}=\text { constant }=\mathbf{H}
$$

Equation (3.4) shows us that the energy per unit of mass is constant, with the first term on the left-hand side being the kinetic energy and the second term the potential energy. Equation (3.5) indicates that also the angular momentum is constant. Rewriting this formula together with equation (3.3) to polar coordinates, it can be solved analytically, resulting in the following orbital equation [Wakker, $1997 a]$

$$
r=\frac{H^{2} / \mu}{1+c \cos (\varphi-\omega)}
$$

with $r$ and $\varphi$ the polar coordinates and $c$ and $\omega$ constants of integration. The shape of equation (3.6) resembles the formula of a conic section in polar coordinates, being

$$
r=\frac{p}{1+e \cos \theta}
$$

with $p$ the semi-latus rectum, $e$ the eccentricity and $\theta$ an angle, which will be called the true anomaly (see figure 3.2, left). The eccentricity is equal to the constant ratio of the distance from any point on the conic section to the focus and the distance from that point to the directrix: $e=r / d=p / d_{0}$. Comparing equation (3.6) and (3.7) it follows that

$$
H^{2} / \mu=p \quad ; \quad c=e \quad ; \quad \varphi-\omega=\theta
$$

For $\varphi=\omega$, distance $r$ is minimal. Since $\theta=0$ in that case, $\theta$ is measured from the point where $r$ is minimal. The angle $\omega$ indicates the position where $r$ is minimal, called the periapsis. If the conic section is closed, exactly opposite to the periapsis the apoapsis can be found, where $r$ is maximal. These conic sections representing orbits in space are often called Keplerian orbits, since Johannes Kepler (15711630) was the first to capture the movement of the planets in empirical laws, including that the orbit of a planet around the Sun is (by approximation) an ellipse. This special case of a conic section, an ellipse, holds for $0<e<1$. If

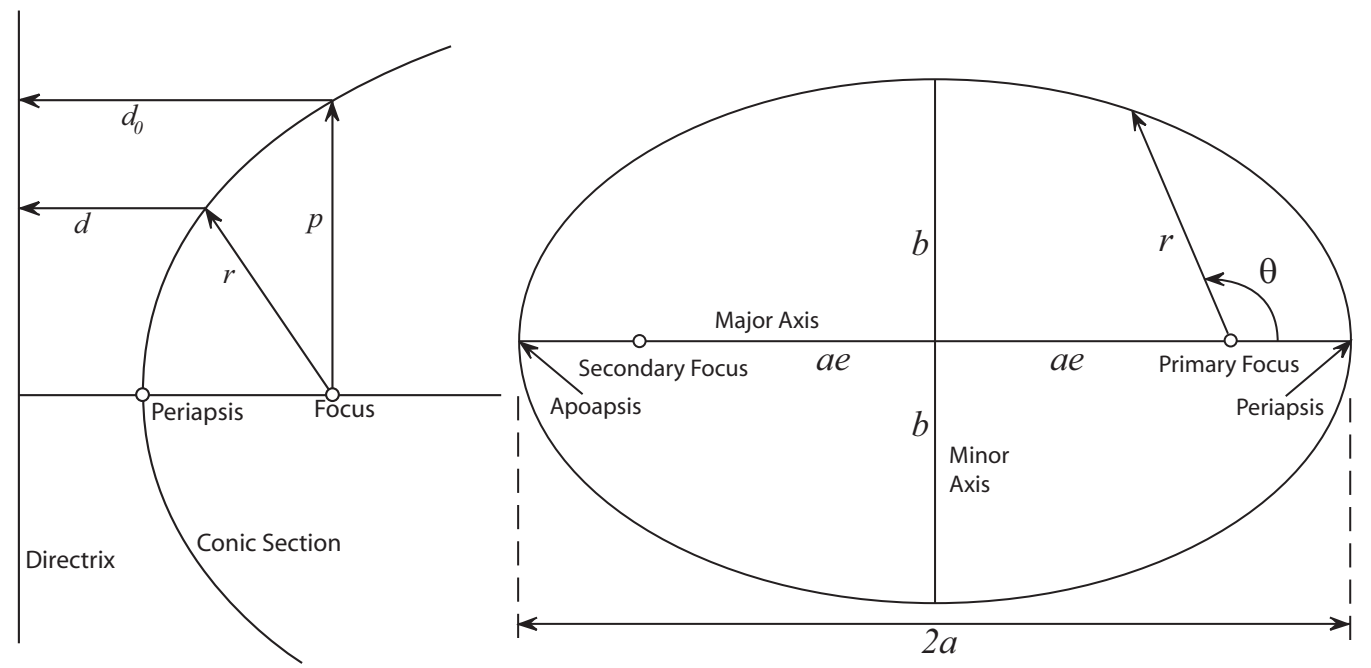

Figure 3.2 The general conic section (left) and a specific case: The ellipse (right) (adapted from [Cornelisse et al., 1979]). 
$e=0, r=p$ and a circle results. For $e=1$ a parabola comes into being, and for $e>1$ a hyperbola results. Since the circle and parabola are more theoretical cases (because the eccentricity will never be exactly equal to 0 or 1 ), only the other two will be discussed. The ellipse will first be discussed (section 3.2.1), followed by the hyperbola (section 3.2.2).

\subsubsection{The Ellipse}

The geometry of an ellipse can be seen in figure 3.2 on the right. The distance from periapsis to apoapsis is $2 a$ with $a$ the semi-major axis. From the geometry and equation (3.7) it follows that

$$
a=\frac{1}{2}\left(r_{p}+r_{a}\right)=\frac{1}{2}\left(\frac{p}{1+e}+\frac{p}{1-e}\right)=\frac{p}{1-e^{2}}
$$

The distance from a focus to the center is found to be $f=a-r_{p}=a e$. For the semi-minor axis $b$ it can be found that $b=a \sqrt{1-e^{2}}$. Now the geometry is completely defined and the motion can be determined.

For the total energy it can be derived that $\mathcal{E}=-\mu / 2 a$ [Wakker, 1997a]. Together with equation (3.4) it results in the following equation for the velocity

$$
V^{2}=\mu\left(\frac{2}{r}-\frac{1}{a}\right)
$$

From this equation it follows that the velocity is maximal in periapsis and minimal in apoapsis.

$$
V_{p}^{2}=\frac{\mu}{a}\left(\frac{1+e}{1-e}\right) \quad ; \quad V_{a}^{2}=\frac{\mu}{a}\left(\frac{1-e}{1+e}\right)
$$

The total time needed to complete one revolution was first found empirically by Kepler and later on confirmed analytically with Newton's laws of motion, giving

$$
T=2 \pi \sqrt{a^{3} / \mu}
$$

This concludes the basic description of an ellipse. To go to a hyperbola, the conic must be 'opened'. The parabola is the first open curve when increasing $e$ monotonically. In this case $e=1$ and since $p$ must be finite, it follows from equation (3.9) that $a=\infty$. Equation (3.10) was derived in a general way and thus also holds for parabolae and hyperbolae. Substituting $a=\infty$ results in

$$
V_{e s c}=\sqrt{\frac{2 \mu}{r}}
$$

This is the so-called escape velocity; the velocity needed to escape an attracting body. At an even higher velocity one enters a hyperbola.

\subsubsection{The Hyperbola}

In the case of a hyperbola $e>1$, so the denominator on the right-hand side of equation (3.7) is negative for $\cos \theta<-e^{-1}$. Furthermore $p$ must be a real positive number as it is equal to $H^{2} / \mu$, and also $r$ must be real and positive to make any 
sense. From this it follows that the line making an angle of $\theta=\arccos \left(-e^{-1}\right)$ with the symmetry axis is an asymptote of the hyperbola. From a purely mathematical point of view there is also another branch of the hyperbola on the other side of the directrix. But since gravity is an attracting force this does not have any physical meaning. The semi-major axis is negative in the case of a hyperbola, following from equation (3.9). In this way many of the earlier derived equations are still valid, like equation (3.10). From this equation it follows that the velocity takes a maximum value at the periapsis and a minimum value for $r \rightarrow \infty$

$$
V_{p}^{2}=\frac{\mu}{-a}\left(\frac{e+1}{e-1}\right) \quad ; \quad V_{\infty}^{2}=-\frac{\mu}{a}
$$

Thus at an infinite distance there is still a relative velocity between the two bodies. This is called the hyperbolic excess velocity. When combining equation (3.14) with equations (3.10) and (3.13) one obtains

$$
V^{2}=V_{e s c}^{2}+V_{\infty}^{2}
$$

So, at any point in the hyperbolic trajectory, the local velocity can be expressed in terms of local escape velocity and hyperbolic excess velocity. The dependence of velocity on distance for a hyperbolic orbit around the Earth is shown in figure 3.3 for three values of the eccentricity. The assumption is made that the perigee is at $500 \mathrm{~km}$ above the Earth's surface. Also shown in this figure is a curve representing the local escape velocity. Remarkably a relatively small velocity increase above the escape velocity at $500 \mathrm{~km}$ results in rather large values of $V_{\infty}$. Secondly it can be concluded that at a distance of around $400,000 \mathrm{~km}$, the difference between $V$ and $V_{\infty}$ is small. This feature is of utmost importance in the calculation of interplanetary trajectories.

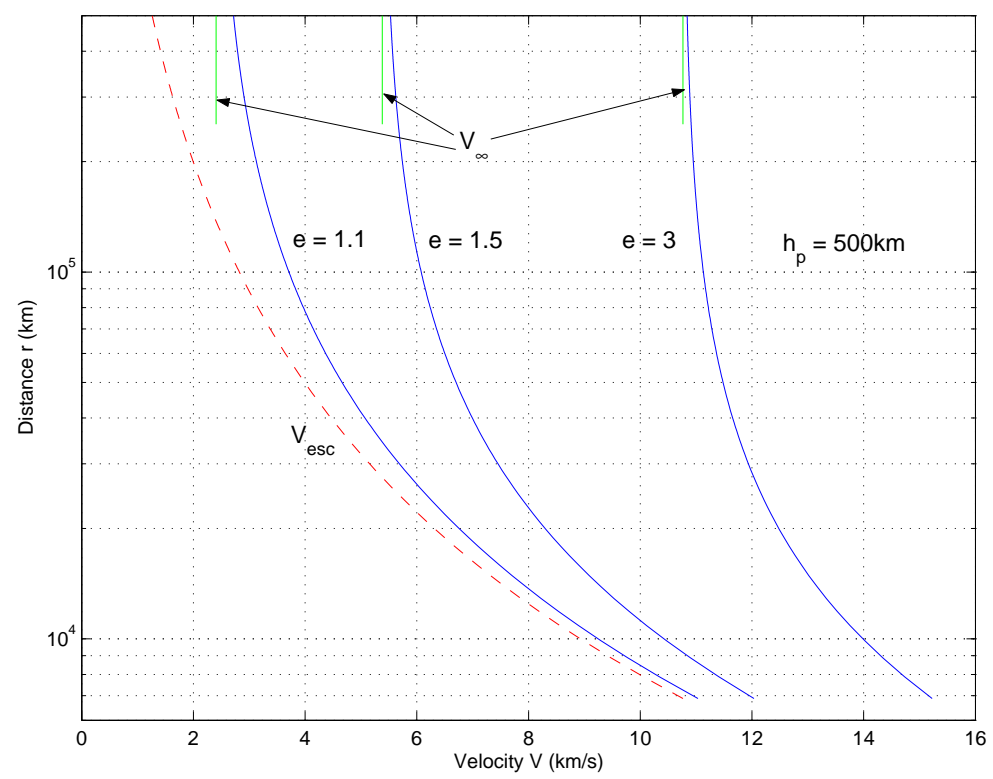

Figure 3.3 Dependence of velocity on distance for three hyperbolic trajectories. The lower right boundary indicates the perigee conditions. 


\subsection{Third-Body Effects (Qualitative)}

The Keplerian orbits discussed in the previous subsection are only valid for a twobody system. In reality there are multiple bodies to take into account. But it will be shown that in most cases the Keplerian orbits are an accurate description of the actual orbits, with other bodies causing just minor disturbances.

Equation (3.2) shows that the bodies that are close to body $i$ and have a large mass, have the largest influence on body $i$. This equation cannot be used directly, since it describes the movement of the bodies with respect to the common center of gravity, the barycenter. If it is rewritten to having the origin at body $k$ one finds [Wakker, 1997a]

$$
\ddot{\mathbf{r}}_{i}=-G \frac{m_{i}+m_{k}}{r_{i}^{3}} \mathbf{r}_{i}+G \sum_{j=1}^{n *} m_{j}\left(\frac{\mathbf{r}_{j}-\mathbf{r}_{i}}{r_{i j}^{3}}-\frac{\mathbf{r}_{j}}{r_{j}^{3}}\right)
$$

The vectors are with respect to body $k$. This equation describes the movement of body $i$ with respect to a non-rotating reference frame with body $k$ as origin, under the influence of the attractive forces of all bodies in the system. The first term on the right-hand side represents the main acceleration $a_{m}$ and the second term is the disturbance acceleration $a_{d}$. Why this second term can be called a disturbance will be shown now.

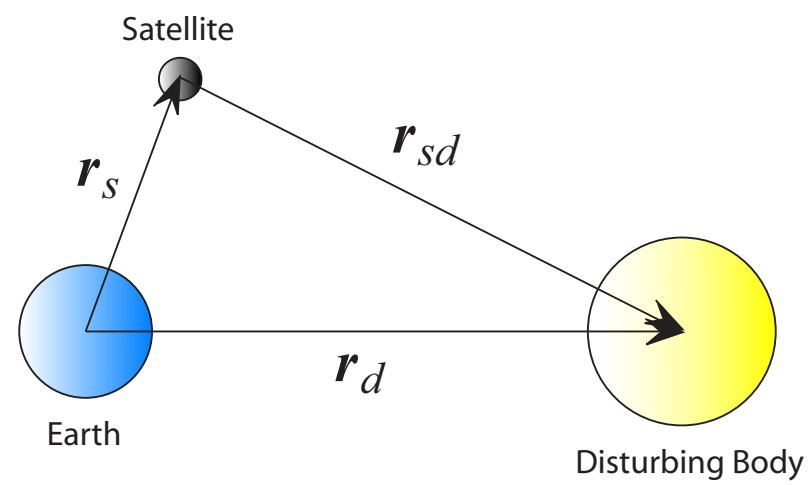

Figure 3.4 Relative positions of three bodies.

When equation (3.16) is applied for a satellite revolving around the Earth and considering just one disturbing body (see figure 3.4), it follows that [Wakker, $1997 a]$

$$
\ddot{\mathbf{r}}_{s}=-G \frac{m_{E}}{r_{s}^{3}} \mathbf{r}_{s}+G m_{d}\left(\frac{\mathbf{r}_{s d}}{r_{s d}^{3}}-\frac{\mathbf{r}_{d}}{r_{d}^{3}}\right)
$$

with $m_{E}$ the mass of the Earth, $\mathbf{r}_{s}$ and $\mathbf{r}_{d}$ the position vector of the satellite and the disturbing body respectively with respect to the Earth, and $\mathbf{r}_{s d}$ the position vector from the satellite to the disturbing body. If it is assumed that $r_{s} / r_{d} \ll 1$ it can be found for the maximum relative disturbance acceleration [Wakker, 1997a]

$$
\frac{a_{d}}{a_{m}}=2 \frac{m_{d}}{m_{E}} \frac{r_{s}^{3}}{r_{d}^{3}}
$$


This maximum disturbance occurs when the three bodies are aligned. For the Sun and the Moon this relative disturbance acceleration is found to be $1.6 \cdot 10^{-5}$ and $3.3 \cdot 10^{-5}$ respectively, for a satellite at a geostationary altitude. Due to the large mass of the Sun and the proximity of the Moon these are the least small disturbances due to third-body effects. These values show that indeed one can speak of disturbances.

When the spacecraft moves further away from the Earth and closer to other bodies, the effect of the other bodies will increase, until the gravity of this other body will prevail. Then it is more logical to describe the movement of the spacecraft with respect to this other body. The question now arises when exactly this takes place. For this purpose the so-called sphere of influence is introduced. Inside this region, the spacecraft is in orbit around the particular planet rather than the Sun for example. As a definition, on this sphere of influence the relative disturbance acceleration with respect to the first body is equal to that of the other body: $\left(a_{d} / a_{m}\right)_{1}=\left(a_{d} / a_{m}\right)_{2}$. Then we have equal right to approximate the trajectory by a disturbed two-body orbit around the Earth or the Sun. The following assumptions are used to obtain a simple expression for the radius of this sphere of influence:

- The distance from the planet to the spacecraft is much smaller than the distance from the planet to the disturbing body: $r_{s} \ll r_{d}$. (This is quite realistic for the case that the disturbing body is the Sun, as will be shown)

- The mass of the spacecraft is negligible with respect to that of both other bodies.

Using these assumptions it follows for the radius of the sphere of influence [Wakker, 1997a]

$$
r_{S o I}=r_{d}\left(\frac{m_{p l}}{m_{S}}\right)^{2 / 5}
$$

The mass of the planet $m_{p l}$ and that of the Sun $m_{S}$ can also be replaced by the mass of a moon and its parenting planet respectively. It must be noted that the mentioned assumptions force the sphere of influence to be a perfect sphere. In reality it is not a perfect sphere, although resembling one. The radii of the spheres of influence of some selected planets and moons can be found in table 3.1; the planets with respect to the Sun and the moons with respect to their parenting planet. Both the absolute value and the value relative to the semi-major axis are given. This semi-major axis is taken to represent an averaged $r_{d}$.

\subsection{Planetary Swing-By}

The planets in our solar system can be used to our advantage: their gravity can assist in interplanetary flights. In short it comprises of an exchange of momentum between the spacecraft and the planet. Since this is not a satisfactory explanation a more thorough one will follow now. 


\begin{tabular}{|c|r|r|}
\hline & \multicolumn{2}{|c|}{ Sphere of Influence } \\
Planet/Moon & $\left(10^{6} \mathrm{~km}\right)$ & $\left(10^{-2} \mathrm{a}\right)$ \\
\hline Venus & 0.616 & 0.57 \\
Earth & 0.925 & 0.62 \\
Moon & 0.0662 & 17.2 \\
Mars & 0.577 & 0.25 \\
Jupiter & 48.2 & 6.19 \\
Saturn & 54.6 & 3.82 \\
Uranus & 51.8 & 1.80 \\
Neptune & 86.6 & 1.93 \\
Triton & 0.0120 & 3.37 \\
\hline
\end{tabular}

Table 3.1 Spheres of influence for selected planets (w.r.t. Sun) and moons (w.r.t. parenting planet).

During an interaction between a planet and a spacecraft the momentum is conserved

$$
m_{s} \mathbf{V}_{s_{i}}+m_{t} \mathbf{V}_{t_{i}}=m_{s} \mathbf{V}_{s_{f}}+m_{t} \mathbf{V}_{t_{f}}
$$

with $m_{s}$ the mass of the satellite, $m_{t}$ the mass of the target planet, $\mathbf{V}_{s}$ the satellite's velocity, and $\mathbf{V}_{t}$ the velocity of the target planet, both with respect to an inertial space frame. Subscripts $i$ and $f$ represent initial and final conditions respectively. Equation (3.20) can be rewritten as

$$
\mathbf{V}_{t_{f}}-\mathbf{V}_{t_{i}}=\frac{m_{s}}{m_{t}}\left(\mathbf{V}_{s_{i}}-\mathbf{V}_{s_{f}}\right)
$$

Since $m_{s}$ is typically in the order of $10^{3} \mathrm{~kg}$, while $m_{t}$ is in the range of $10^{24}-10^{27}$ $\mathrm{kg}$, the indicated mass fraction is approximately zero: $m_{s} / m_{t} \approx 0$. Hence it can be assumed that $\mathbf{V}_{t_{f}}=\mathbf{V}_{t_{i}}$. For example, Galileo slowed the Earth down by only $0.1 \mathrm{~nm}$ per year $[N A S A / J P L, 2005 a]$.

A plot of the generic geometry is given in figure 3.5. The figure is in the plane spanned by the incoming and outgoing hyperbolic excess velocity $\left(\mathbf{V}_{\infty_{i}}\right.$ and $\mathbf{V}_{\infty_{f}}$, respectively). $\mathbf{V}_{t}^{\prime}$ gives the component of the planet's velocity $\mathbf{V}_{t}$ in this plane. The velocities can be taken out from this figure and put in a vector diagram (see figure 3.6). The nomenclature will be made clear throughout the text. From this figure the velocity of the satellite with respect to the planet can be established. Taken at a sufficiently large distance this velocity is equal to the hyperbolic excess velocity $\mathbf{V}_{\infty}=\mathbf{V}_{s}-\mathbf{V}_{t}$. Applying energy conservation in the planet reference frame results in the notion that, although the direction is different, the magnitudes of $\mathbf{V}_{\infty_{i}}$ and $\mathbf{V}_{\infty_{f}}$ are the same. The figure shows that if the satellite passes behind the planet, a modest gravitational deflection aligns $\mathbf{V}_{\infty}$ more with $\mathbf{V}_{t}$ and thus $\left|\mathbf{V}_{s_{f}}\right|>\left|\mathbf{V}_{s_{i}}\right|$; the spacecraft accelerates. Passing in front of the planet the opposite takes place, so the spacecraft slows down.

Reconsidering figure 3.5 the impact parameter $B$ is defined as the perpendicular distance from the target planet to the incoming hyperbolic leg. Conservation of momentum gives

$$
B V_{\infty}=r_{p} V_{p}
$$




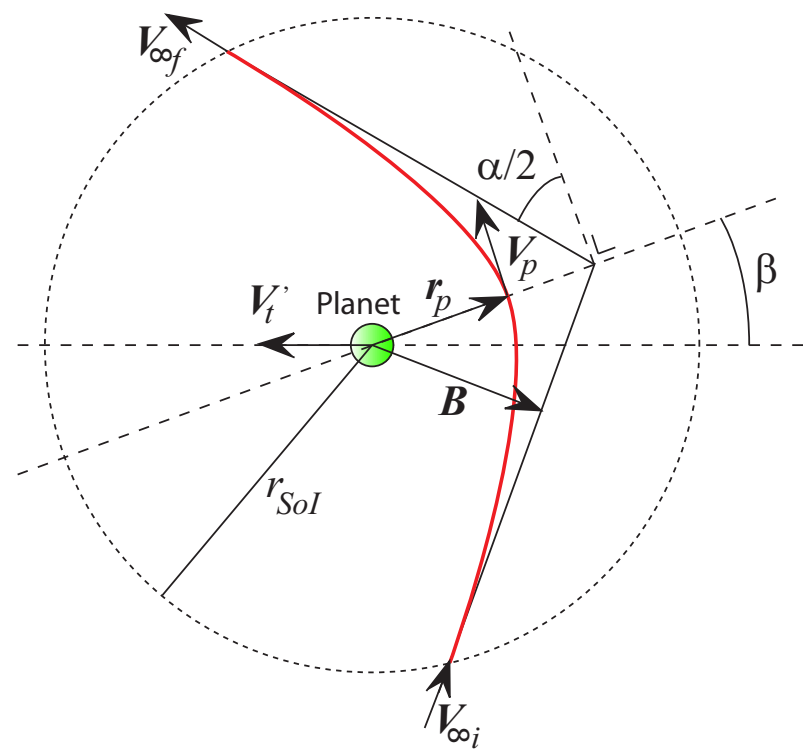

Figure 3.5 The in-plane geometry of hyperbolic encounter trajectories [Cornelisse et al., 1979].

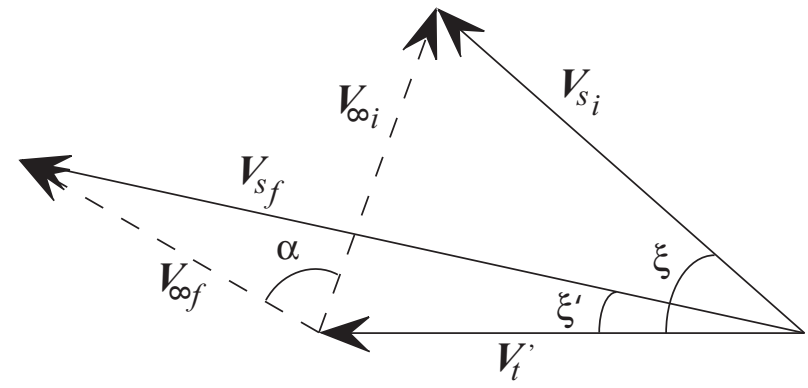

Figure 3.6 Vector diagram of velocities of the gravity assist from figure 3.5.

Using equation (3.15) the following equation for the perigee distance $r_{p}$ can be found [Cornelisse et al., 1979]

$$
r_{p}=-\frac{\mu_{t}}{V_{\infty}^{2}}+\sqrt{\frac{\mu_{t}^{2}}{V_{\infty}^{4}}+B^{2}}
$$

Evaluating expression (3.7) at infinite distance from the planet gives

$$
\cos \left(\frac{\pi}{2}+\frac{\alpha}{2}\right)=-\frac{1}{e}
$$

With equation (3.14) and the geometrical relations for a hyperbola it follows that

$$
\sin \frac{\alpha}{2}=\frac{1}{e}=\frac{1}{1+\frac{r_{p} V_{\infty}^{2}}{\mu_{t}}}=\frac{1}{\sqrt{1+\frac{B^{2} V_{\infty}^{4}}{\mu_{t}^{2}}}}
$$

From this equation it can be seen that if the flyby is closer (smaller $B$ ), the bending angle $\alpha$ is larger. Also it can be seen from this formula that $\alpha$ is larger for heavier planets, and lower $V_{\infty}$. If the radius $r_{p}$ is equal to or less than the planet's radius $R_{p l}$, the spacecraft will impact on the planet's surface. From equation (3.23) the condition to prevent impact is

$$
\sqrt{1+\frac{B^{2} V_{\infty}^{4}}{\mu_{t}^{2}}} \geq \frac{V_{\infty}^{2} R_{p l}}{\mu_{t}}+1
$$


or with the expression for the escape velocity at the planet's surface, $V_{e s c_{s}}=$ $\sqrt{2 \mu_{t} / R_{p l}}$

$$
B \geq R_{p l} \sqrt{1+\frac{V_{e s c_{s}}^{2}}{V_{\infty}^{2}}}
$$

The expression on the right-hand side determines for each value of $V_{\infty}$ the so-called capture radius $r_{\text {cap }}$. When the incoming asymptote crosses a sphere with radius $r_{c a p}$ around the planet, the spacecraft will impact somewhere on the planet. This restriction must be kept in mind when designing a gravity assist. The requirement becomes even more stringent when taking into account the environment of a planet. Severe radiation or unwanted atmospheric drag can cause a significant degradation of the trajectory and/or the satellite. As a baseline the smallest swing-by distances of previous and current missions are taken to define the smallest allowable periapsis distance $r_{p, \text { limit }}$. The values are shown in table 3.2 and come from the literature study by Schlijper [2002], except for the height of the Mars swing-by of the Rosetta mission in February of 2007 [ESA, 2006b]. In some cases, one probably could get closer than is already done (e.g. Uranus, Voyager 2) or will be done (e.g. New Horizons, Pluto), but as a baseline these numbers can serve well.

\begin{tabular}{|l|c|c|c|l|}
\hline Planet & $R_{p l}[\mathrm{~km}]$ & $r_{p, \text { limit }}\left[R_{p l}\right]$ & $h_{p, \text { limit }}[\mathrm{km}]$ & Mission \\
\hline Mercury & 2440 & 1.082 & 200 & Messenger \\
Venus & 6052 & 1.047 & 284 & Cassini \\
Earth & 6378 & 1.048 & 306 & Galileo \\
Mars & 3397 & 1.076 & 257 & Rosetta \\
Jupiter & 71492 & 1.60 & 42895 & Pioneer 11 \\
Saturn & 60268 & 1.342 & 20612 & Pioneer 11 \\
Uranus & 25559 & 4.190 & 81533 & Voyager 2 \\
Neptune & 24764 & 1.181 & 4482 & Voyager 2 \\
Pluto & 1151 & 9.34 & 9600 & New Horizons \\
\hline
\end{tabular}

Table 3.2 Limits on periapsis distance for each solar system planet expressed in their radius [NASA/JPL, 2006c].

Finally in this section it is determined which planets are preferable for a swingby. To that end an expression is derived for the change of the total heliocentric energy per unit of mass:

$$
\Delta \mathcal{E}=\frac{1}{2}\left(V_{s_{f}}^{2}-V_{s_{i}}^{2}\right)=\frac{1}{2}\left(\mathbf{V}_{s_{f}}+\mathbf{V}_{s_{i}}\right) \cdot\left(\mathbf{V}_{s_{f}}-\mathbf{V}_{s_{i}}\right)
$$

with $\mathbf{V}_{s_{i}}=\mathbf{V}_{\infty_{i}}+\mathbf{V}_{t}$ and $\mathbf{V}_{s_{f}}=\mathbf{V}_{\infty_{f}}+\mathbf{V}_{t}$ as earlier defined in this chapter, and bearing in mind that the inner product of two perpendicular vectors is zero, it follows that

$$
\Delta \mathcal{E}=\mathbf{V}_{t}^{\prime} \cdot\left(\mathbf{V}_{\infty_{f}}-\mathbf{V}_{\infty_{i}}\right)
$$

which can, according to figure 3.5 and using equation (3.25), be written as

$$
\Delta \mathcal{E}=\frac{2 V_{t}^{\prime} V_{\infty} \cos \beta}{\sqrt{1+\frac{B^{2} V_{\infty}^{4}}{\mu_{t}^{2}}}}
$$

For a particular planet, the value of $\Delta \mathcal{E}$ depends on the trajectory parameters $V_{\infty}$, $\beta$ and $B$. For a specified value of $V_{\infty}$, the 'free' energy supplied by the gravity 
assist is maximal for $\beta=0$ and $B=r_{c a p}$. Use is made of the planet's radius $R_{p l}$ and not the limit on periapsis distance $r_{p, l i m}$ from table 3.2. Substituting the value for $\beta$ and the expression for $r_{c a p}$ from equation (3.27) gives

$$
\Delta \mathcal{E}_{\text {max }}=\frac{2 V_{t}^{\prime} V_{\infty}}{1+\frac{R_{p l} V_{\infty}^{2}}{\mu_{t}}}
$$

This expression is plotted for some relevant planets in figure 3.7. From this figure it follows that Jupiter and Saturn are attractive swing-by planets. This is caused by their large mass. As for the inner planets, Venus, but also the Earth is attractive to fly by. Although their masses are relatively low, the orbital velocity around the Sun is relatively large and the radius is relatively small, causing the energy change to be significant. It might appear quite strange to use the Earth as a swing-by planet, since it is the point of departure. But by first flying somewhere else and then coming back, a considerable energy increase can be obtained. For example, both Galileo and Cassini used the Earth [NASA/JPL, 2005a, 2006a].

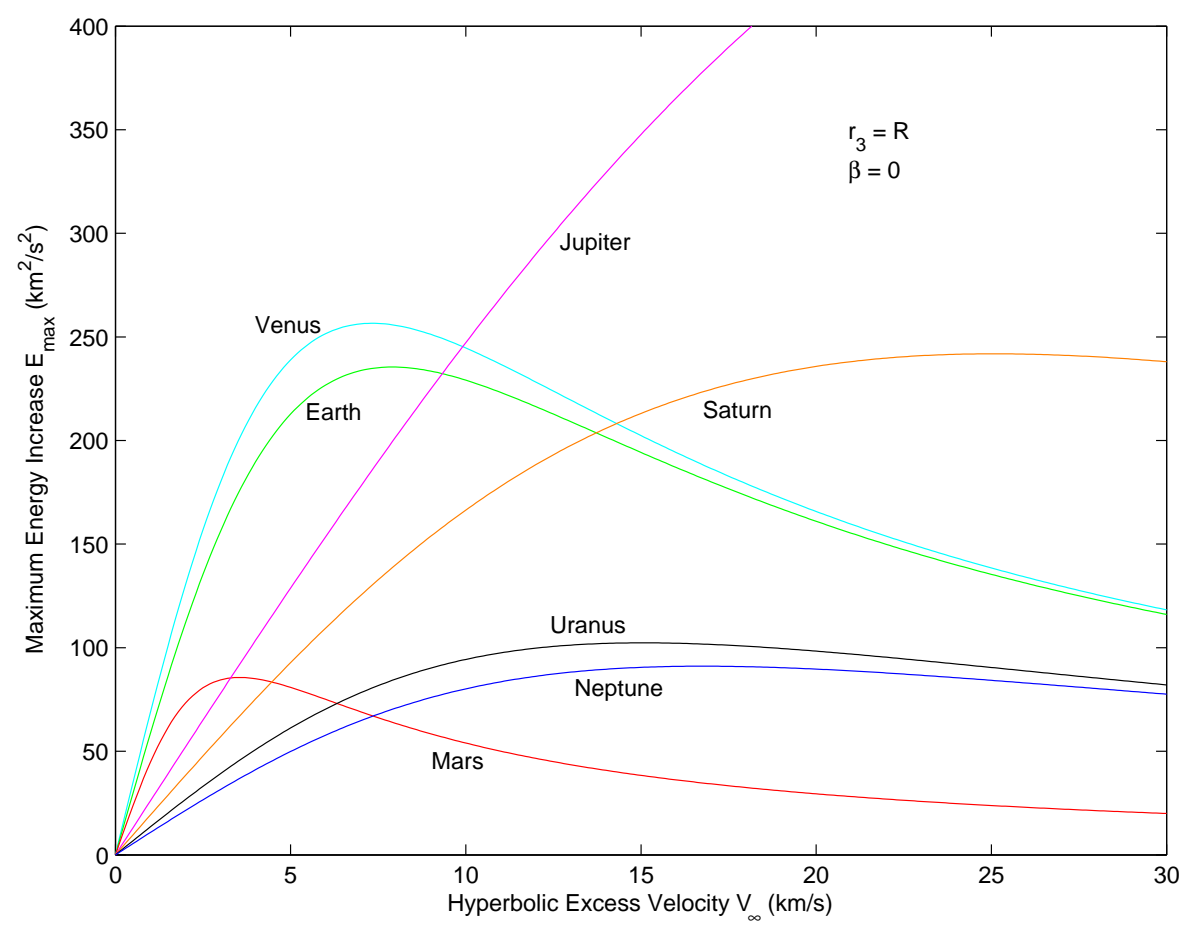

Figure 3.7 The maximum heliocentric energy increase for gravity assists. 

Chapter 4

\section{Perturbations}

In chapter 3 orbits were described as they result from the gravitational attraction of one radially symmetrical body. Although this gives a very good approximation to reality, there are numerous perturbations which should be noted. These will be the point of discussion in this chapter. First the earlier assumption of radially symmetric bodies is tested in section 4.1. Then the effects of the atmosphere will be dealt with in section 4.2 , followed by another check of the third-body effects in section 4.3 , but now in a more quantitative sense. Radiation can cause a pressure and thus a perturbing force as can be read in section 4.4. Also the magnetic field can cause disturbances, see section 4.5. The shortcomings of Newtonian Mechanics is the last mentioned limitation, and is treated in section 4.6. A brief discussion about the implications of these perturbations will close this chapter in section 4.7.

\subsection{Gravity Field Perturbations}

If a body is radially symmetric, for gravitational purposes it can be seen as a point mass. Unfortunately for the ones who favor simplicity, this is not the case. In a first approximation it is, but by determining the polar and equatorial radius already the flattening of the Earth becomes apparent, which is certainly not the only deviation from a perfect sphere. The expression used for the gravity potential thus far is $U=-\mu / r$, consistent with equation (3.1). But from potential theory follows a much more extensive gravity potential of a body with an arbitrary mass distribution in a point outside that body [Wakker, 1997b]:

$$
U=-\frac{\mu}{r}\left[1-\sum_{n=2}^{\infty} \sum_{m=0}^{n} J_{n}^{m}\left(\frac{R}{r}\right)^{n} P_{n}^{m}(\sin \delta)\left\{\cos m\left(\lambda-\lambda_{n}^{m}\right)\right\}\right]
$$

In this equation $r, \delta$, and $\lambda$ are the spherical coordinates of the considered point with respect to a bodycentric with the body rotating reference frame (see figure 4.1): $r$ the distance from the center of mass, $\delta$ the bodycentric latitude, positive to the North from the equator, and $\lambda$ the bodycentric longitude, positive to the East from some reference meridian, the Greenwich meridian in case of the Earth. $R$ is the averaged equatorial body radius. The factors $J_{n}^{m}$ and $\lambda_{n}^{m}$ are constants of the gravitational field; together with $\mu$ and $R$ they constitute the system of constants that is specified by gravity models. The term $P_{n}^{m}(\sin \delta)$ is the Legendre 


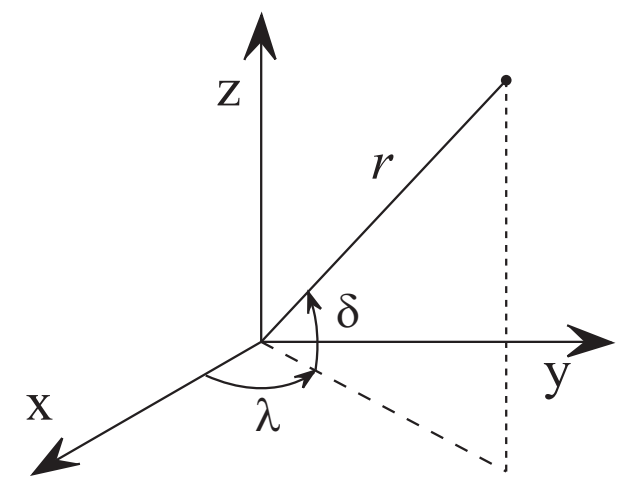

Figure 4.1 Definition of spherical coordinates: distance $r$, latitude $\delta$, and longitude $\lambda$.

function of the first kind which can be written as

$$
P_{n}^{m}(x)=\frac{\left(1-x^{2}\right)^{m / 2}}{2^{n} n !} \frac{\mathrm{d}^{m+n}}{\mathrm{~d} x^{m+n}}\left(x^{2}-1\right)^{n}
$$

in which $x$ represents $\sin \delta$. In equation (4.1) the gravity potential as it has been used before can be recognized as the first term. As can be expected and can be seen from equation (4.1) the influence of these perturbing forces is larger when the point, the satellite in a practical case, is closer to the body. For the coefficient of the first perturbing term of the Earth it is found that $\mathrm{J}_{2}=1.083 \cdot 10^{-3}$, while the other J-coefficients are $2.5 \cdot 10^{-6}$ at the most [Noomen et al., 2003]. Because it is the largest disturbance term, this so-called $\mathrm{J}_{2}$-effect, representing the flattening of the Earth, will now be studied up close. It can be derived that the disturbing accelerations due to this term are [Wakker, 1997b]

$$
\begin{aligned}
& a_{r}=\frac{3}{2} \mu_{E} J_{2} \frac{R_{E}^{2}}{r^{4}}\left(3 \sin ^{2} \delta-1\right) \\
& a_{\delta}=-\frac{3}{2} \mu_{E} J_{2} \frac{R_{E}^{2}}{r^{4}} \sin 2 \delta
\end{aligned}
$$

For a satellite at an altitude of $500 \mathrm{~km}$, the maximum values of these accelerations are $\left|a_{r}\right|_{\max }=2\left|a_{\delta}\right|_{\max }=23.5 \mathrm{~mm} / \mathrm{s}^{2}$ which is about $0.3 \%$ of the local gravitational acceleration. Other planets are also flattened, giving rise to this same $\mathrm{J}_{2}$-effect. Saturn has the largest flattening, as can be seen in the overview of table 4.1. Also the disturbance accelerations are included, evaluated at a distance of 1.1 planet radii.

\begin{tabular}{|l|c|c|c|}
\hline Planets & $\mathrm{J}_{2}$ & $a_{d}\left[\mathrm{~mm} / \mathrm{s}^{2}\right]$ & $\Delta \Omega$ per orbit \\
\hline Mercury & $6.0 \cdot 10^{-5}$ & 0.457 & $-0.0268^{\circ}$ \\
Venus & $4.46 \cdot 10^{-6}$ & 0.0810 & $-0.0020^{\circ}$ \\
Earth & $1.08 \cdot 10^{-3}$ & 21.7 & $-0.483^{\circ}$ \\
Mars & $1.96 \cdot 10^{-3}$ & 14.9 & $-0.875^{\circ}$ \\
Jupiter & $1.47 \cdot 10^{-2}$ & 751 & $-6.58^{\circ}$ \\
Saturn & $1.63 \cdot 10^{-2}$ & 348 & $-7.27^{\circ}$ \\
Uranus & $3.34 \cdot 10^{-3}$ & 60.7 & $-1.49^{\circ}$ \\
Neptune & $3.41 \cdot 10^{-3}$ & 80.8 & $-1.52^{\circ}$ \\
\hline
\end{tabular}

Table $4.1 \mathrm{~J}_{2}$-Terms of the planets [NASA/GSFC, 2006] and accompanying disturbances at 1.1 planet radii. 
The influence of the $\mathrm{J}_{2}$-effect can be further understood when next to the magnitude also the direction is studied. It is found that for the change in orbital elements after one revolution holds [Wakker, 1997b]

$$
\begin{aligned}
& \Delta a_{2 \pi}=\Delta e_{2 \pi}=\Delta i_{2 \pi}=0 \\
& \Delta \Omega_{2 \pi}=-3 \pi J_{2}\left(\frac{R}{p}\right)^{2} \cos i \\
& \Delta \omega_{2 \pi}=-\frac{3}{2} \pi J_{2}\left(\frac{R}{p}\right)^{2}\left(5 \cos ^{2} i-1\right)
\end{aligned}
$$

From equations (4.5) it is apparent that there are no secular variations in the size of the orbit, but there are in the orientation of the orbit. The decrease per orbit in right ascension of the ascending node $\Omega$ of a satellite at 1.1 planet radii can be found in table 4.1 .

When a satellite is in orbit around a particular planet it seems to make sense to take at least the $\mathrm{J}_{2}$-effect into account. Once in orbit around Neptune this effect cannot be neglected, especially since the flattening of Neptune is about 6 times larger than the flattening of the Earth, as mentioned in section 2.1, which is also qualitatively reflected in the value of $\mathrm{J}_{2}$. During gravity assists planets could be flown by rather close. But the number of revolutions during a gravity assist has a theoretical upper limit of 0.5 (see equation (3.25)), which in practice will even never be attained. Thus, the gravity field perturbations can safely be neglected, due to the lack of time for the disturbance to affect the orbit.

\subsection{Atmospheric Drag}

The atmosphere can also deviate the orbits from the perfect conical section. This will been shown in fact in the section on aerogravity assists, section 6.4. But in that case it is not an unwanted side-effect, on the contrary. It is used to enhance the performance, by shortening the required time and/or the needed fuel. On the other hand, during ascent from the Earth, atmospheric drag plays an important role. Since this will be counter-acted by the launcher, no further attention is paid to it, also because in the performance figures in section 5.3 this effect has already been taken into account. The other parts in atmospheres are the aerogravity assists and aerocapture. These will be discussed in section 6.4 which is deemed to be sufficient.

\subsection{Third-Body Effects (Quantitative)}

Third-body effects have already been discussed in section 3.3, but these were the general principles behind it. Now some numbers will be attached to it. As a numerical example the Jupiter flyby by Cassini-Huygens will be taken. Cassini's closest approach to Jupiter was at a distance of $10 \cdot 10^{6} \mathrm{~km}$ [NASA/JPL, 2006a]. It should be clear that this is in fact relatively close to Jupiter, keeping in mind that the sphere of influence of Jupiter has a radius of $48.2 \cdot 10^{6} \mathrm{~km}$. Therefore the disturbance accelerations will be relatively large. Then for this configuration and using equation (3.18) the maximum relative disturbance accelerations can be 
found, shown in table 4.2. For the distances to the disturbing body the minimum distances are taken: for the inner planets this occurs during inferior conjunction and for the outer planets during opposition. Also the four Galilean Moons are represented. The accompanying accelerations cannot be computed using equation (3.18), since the assumption $r_{s} / r_{d} \ll 1$ does not hold (see the third column of table 4.2). Therefore the second term of the right-hand side of equation (3.17) must be used, which is for convenience restated here.

$$
a_{d}=G m_{d}\left(\frac{\mathbf{r}_{s d}}{r_{s d}^{3}}-\frac{\mathbf{r}_{d}}{r_{d}^{3}}\right)
$$

It might look strange that the moon Io causes a larger disturbance acceleration than the other Galilean moons. Since Io is furthest away from the satellite it would be expected the other way around. But then again, Io is closer to Jupiter, and thus has a larger influence on Jupiter. Since the net disturbance acceleration is with respect to the center of Jupiter, this significantly enlarges the disturbance. It can also be noted that both Io, Europa and Ganymede have a larger gravitational influence on the satellite than the Sun.

\begin{tabular}{|l|c|c|c|c|}
\hline Disturbing Body & $m_{d} / m_{a}$ & $r_{d} / r_{s}$ & $a_{d} / a_{m}$ & $a_{d}\left[\mathrm{~mm} / \mathrm{s}^{2}\right]$ \\
\hline Sun & $1.05 \cdot 10^{3}$ & 77.8 & $4.44 \cdot 10^{-3}$ & $5.63 \cdot 10^{-3}$ \\
Venus & $2.56 \cdot 10^{-3}$ & 67.0 & $1.70 \cdot 10^{-8}$ & $2.16 \cdot 10^{-8}$ \\
Earth & $3.15 \cdot 10^{-3}$ & 62.9 & $2.53 \cdot 10^{-8}$ & $3.21 \cdot 10^{-8}$ \\
Mars & $3.38 \cdot 10^{-4}$ & 55.0 & $4.05 \cdot 10^{-9}$ & $5.14 \cdot 10^{-9}$ \\
Saturn & $3.00 \cdot 10^{-1}$ & 64.9 & $2.19 \cdot 10^{-6}$ & $2.78 \cdot 10^{-6}$ \\
Uranus & $4.57 \cdot 10^{-2}$ & 209 & $9.98 \cdot 10^{-9}$ & $1.27 \cdot 10^{-8}$ \\
Neptune & $5.39 \cdot 10^{-2}$ & 372 & $2.10 \cdot 10^{-9}$ & $2.66 \cdot 10^{-9}$ \\
Io & $4.70 \cdot 10^{-5}$ & 0.96 & $2.65 \cdot 10^{-2}$ & $3.36 \cdot 10^{-2}$ \\
Europa & $2.56 \cdot 10^{-5}$ & 0.93 & $5.73 \cdot 10^{-3}$ & $7.26 \cdot 10^{-3}$ \\
Ganymede & $7.85 \cdot 10^{-5}$ & 0.90 & $6.95 \cdot 10^{-3}$ & $8.81 \cdot 10^{-3}$ \\
Callisto & $5.69 \cdot 10^{-5}$ & 0.81 & $1.70 \cdot 10^{-3}$ & $2.15 \cdot 10^{-3}$ \\
\hline
\end{tabular}

Table 4.2 Relative third-body disturbance accelerations during the Jupiter flyby by Cassini-Huygens.

It is also interesting to look at the third-body behavior when a satellite performs one of the heliocentric legs. For a numerical example a position right in between Uranus and Neptune is taken. Again equation (4.6) is used since $r_{s} / r_{d} \ll 1$ does not hold. The values can be found in table 4.3. The main acceleration by the Sun is $9.78 \cdot 10^{-3} \mathrm{~mm} / \mathrm{s}^{2}$, and the other accelerations are clearly much smaller.

\subsection{Radiation Pressure}

Light can be considered as a stream of photons, each one traveling at the speed of light $c$. They carry an impulse which can be transferred to a spacecraft. The magnitude of the acceleration it causes can be expressed by [Wakker, 1997b]

$$
a=\frac{W}{m c} S\left(1+R_{s}\right) \cos ^{2} \zeta
$$

with $W$ the power density of the bundle photons in $\mathrm{W} / \mathrm{m}^{2}, m$ the mass of the spacecraft, $S$ the surface area of the satellite, $R_{s}$ the reflection coefficient $\left(0<R_{s}<1\right)$ and $\zeta$ the incident angle of the radiation on the surface. The reflection coefficient 


\begin{tabular}{|l|c|c|}
\hline Disturbing Body & $a_{d} / a_{m}$ & $a_{d}\left[\mathrm{~mm} / \mathrm{s}^{2}\right]$ \\
\hline Mercury & $6.72 \cdot 10^{-4}$ & $6.57 \cdot 10^{-6}$ \\
Venus & $2.84 \cdot 10^{-3}$ & $2.78 \cdot 10^{-5}$ \\
Earth & $1.82 \cdot 10^{-3}$ & $1.78 \cdot 10^{-5}$ \\
Mars & $8.46 \cdot 10^{-5}$ & $8.28 \cdot 10^{-7}$ \\
Jupiter & $2.29 \cdot 10^{-2}$ & $2.24 \cdot 10^{-4}$ \\
Saturn & $2.67 \cdot 10^{-3}$ & $2.61 \cdot 10^{-5}$ \\
Uranus & $9.68 \cdot 10^{-4}$ & $9.47 \cdot 10^{-6}$ \\
Neptune & $1.02 \cdot 10^{-3}$ & $9.99 \cdot 10^{-5}$ \\
Pluto & $1.55 \cdot 10^{-8}$ & $1.51 \cdot 10^{-10}$ \\
\hline
\end{tabular}

Table 4.3 Relative third-body disturbance accelerations during a heliocentric leg right between Uranus and Neptune.

\begin{tabular}{|l|c|c|c|c|}
\hline Planet & $R_{p l}$ & $W_{\text {Sun }}\left[\mathrm{W} / \mathrm{m}^{2}\right]$ & $W_{\text {alb }}\left[\mathrm{W} / \mathrm{m}^{2}\right]$ & $W_{\text {th }}\left[\mathrm{W} / \mathrm{m}^{2}\right]$ \\
\hline Mercury & 0.119 & $9.08 \cdot 10^{3}$ & $1.08 \cdot 10^{3}$ & $2.17 \cdot 10^{3}$ \\
Venus & 0.750 & $2.60 \cdot 10^{3}$ & $1.95 \cdot 10^{3}$ & 163 \\
Earth & 0.306 & $1.36 \cdot 10^{3}$ & 416 & 237 \\
Mars & 0.250 & 586 & 147 & 111 \\
Jupiter & 0.343 & 50.3 & 17.2 & 8.30 \\
Saturn & 0.342 & 15.0 & 5.11 & 2.45 \\
Uranus & 0.300 & 3.69 & 1.11 & 0.651 \\
Neptune & 0.290 & 1.51 & 0.437 & 0.267 \\
Pluto & 0.5 & 0.871 & 0.435 & 0.112 \\
\hline
\end{tabular}

Table 4.4 Reflection coefficient and received power density at the planets due to direct sunlight, albedo radiation, and thermal radiation [NASA/GSFC, 2006].

$R_{s}$ depends on the material at the outside of the spacecraft. The limits are total absorption $\left(R_{s}=0\right)$ and perfect reflection $\left(R_{s}=1\right)$. The power density indicates how much radiation is received at the position of the spacecraft. The source of this radiation can be various. The most important source in our solar system is the Sun, radiating energy that originates from internal fusion. The total energy radiated by the Sun equals $3.83 \cdot 10^{26} \mathrm{~W}$ [Carroll and Ostlie, 1996] resulting in a power density of $1360 \mathrm{~W} / \mathrm{m}^{2}$ in the orbit of the Earth. The power density obeys an inverse square law, meaning that at $x$ times the distance the power density has decreased by a factor $x^{2}$. Thus,

$$
W=W_{E}\left(\frac{a_{E}}{r}\right)^{2}
$$

With $r$ the distance between the satellite and the Sun, and $a_{E}$ indicating the Earth's semi-major axis. Other sources of photons can be indirect solar radiation and thermal radiation. Indirect solar radiation simply is solar light reflected by a planetary body surface, atmosphere and/or clouds. It is also known as albedo radiation. The reflection coefficient $R_{p l}$ indicates how much of the incoming radiation is reflected, and the corresponding values for several planets are indicated in table 4.4. The albedo radiation $W_{\text {alb }}$ that follows is also indicated, being the product of the reflectance and the incoming solar power density $W_{S}$. Then finally the thermal radiation. Each body emits radiation corresponding to its own temperature. In table 4.4 also this thermal radiation is included, according to an idealized effective temperature. These power densities can be combined to calculate the disturbance acceleration. The spacecraft will probably not come closer to 
the Sun than Venus (Mercury is not favorable for a swing-by and the heat load is too high), therefore Venus is taken to represent the worst case. Combining the three different power densities results in a value of $4.71 \cdot 10^{3} \mathrm{~W} / \mathrm{m}^{2}$. It must be noted that in general these three will not work in the same direction, but it serves as a worst case. Furthermore, the separate sources do not radiate unidirectional. Especially close to a planet the radiation from the limb of the planet will fall onto the surface under a certain angle, which would decrease the transferred impulse. Again, the above stated value can be used for a worst case scenario. For the mass and dimensions the Galileo spacecraft is taken, to have representative numbers. Then $m=2223 \mathrm{~kg}$ and $S=11 \cdot 5.3=58.3 \mathrm{~m}^{2} \quad[N A S A / J P L, 2005 a]$. For the surface area the magnetic boom length is taken into account, and even exaggerated, again to obtain a worst case value. For the reflection coefficient a value of 1 is taken, corresponding to perfect reflection. The angle of incidence $\zeta$ is taken to be equal to zero, implying normal incidence. Also the last two assumptions result in a worst case situation. Now the disturbance acceleration can be calculated:

$$
a=\frac{4.71 \cdot 10^{3}}{2223 \cdot 2.998 \cdot 10^{8}} 58.3 \cdot(1+1) \cdot \cos ^{2} 0=8.25 \cdot 10^{-4} \mathrm{~mm} / \mathrm{s}^{2}
$$

The gravitational acceleration due to the Sun in Venus' orbit is $11.3 \mathrm{~mm} / \mathrm{s}^{2}$, so one can indeed speak of a disturbance. Now the worst case radiation pressure disturbance acceleration is known, the next disturbance can be dealt with: the electro-magnetic effects.

\subsection{Electro-Magnetic Effects}

Yet another perturbing force is due to the electromagnetic field the planets possess. This electromagnetic field is caused by rapid rotation of the metal(lic) interior of the planet. The first mentioned component for a magnetic field is absent at Mercury, Venus and Pluto, while the second one is not present at Mars. Therefore only the Earth, Jupiter, Saturn, Uranus and Neptune have an appreciable magnetic field [Carroll and Ostlie, 1996]. The magnitudes of their magnetic field is given in table 4.5 .

For the magnetic field to have an effect on the spacecraft, the spacecraft should be electrically charged. This can be the case due to the following. The average thermal velocity of electrons $(\approx 200 \mathrm{~km} / \mathrm{s}$ at $1000 \mathrm{~km}$ altitude $)$ is much larger than the average thermal velocity of the ions $(\approx 10 \mathrm{~km} / \mathrm{s}$ at $1000 \mathrm{~km}$ altitude). Therefore the satellite will have a negative potential of several to several tens of volts with respect to its surroundings [Wakker, 1997b]. The interaction of this moving electric charge with the magnetic field yields a Lorentz force on the satellite. The magnitude of this force is given by

$$
F_{l o r}=q V_{s} B
$$

with $F_{l o r}$ the Lorentz force, $q$ the electric charge, $V_{s}$ the satellite's velocity and $B$ the magnetic field strength. This field strength varies with magnetic latitude and distance. When a dipole (two 'poles' acting as a source and a sink of magnetic energy, respectively) is assumed the variation is as follows [Noomen et al., 2003]

$$
B=B_{0}\left(\frac{R_{p l}}{r}\right)^{3} \sqrt{1+3 \sin ^{2} \Phi}
$$




\begin{tabular}{|c|c|c|c|c|}
\hline \multirow[b]{2}{*}{ Planet } & \multirow[b]{2}{*}{$B_{0}[\mathrm{~T}]$} & $a_{d}\left[\mathrm{~mm} / \mathrm{s}^{2}\right]$ & $a_{d}\left[\mathrm{~mm} / \mathrm{s}^{2}\right]$ & \multirow[b]{2}{*}{$a_{m}\left[\mathrm{~m} / \mathrm{s}^{2}\right]$} \\
\hline & & $\begin{array}{l}\text { At planet's sur- } \\
\text { face, polar }\end{array}$ & $\begin{array}{l}\text { At } 5 \text { planet } \\
\text { radii, equatorial }\end{array}$ & \\
\hline Earth & $3.076 \cdot 10^{-5}$ & $9.36 \cdot 10^{-9}$ & $1.67 \cdot 10^{-11}$ & 9.81 \\
\hline Jupiter & $4.28 \cdot 10^{-4}$ & $6.94 \cdot 10^{-7}$ & $1.24 \cdot 10^{-9}$ & 24.9 \\
\hline Saturn & $2.10 \cdot 10^{-5}$ & $2.03 \cdot 10^{-8}$ & $3.63 \cdot 10^{-11}$ & 10.4 \\
\hline Uranus & $2.28 \cdot 10^{-5}$ & $1.32 \cdot 10^{-8}$ & $2.36 \cdot 10^{-11}$ & 8.86 \\
\hline Neptune & $1.42 \cdot 10^{-5}$ & $9.16 \cdot 10^{-9}$ & $1.64 \cdot 10^{-11}$ & 11.6 \\
\hline
\end{tabular}

Table 4.5 Magnetic field strength at magnetic equator [NASA/GSFC, 2006] and accompanying disturbance accelerations. The main surface gravitational acceleration is indicated in the last column.

with $B_{0}$ the magnetic field strength at the planet's magnetic equator and $\Phi$ the magnetic latitude. The extra identifier 'magnetic' implies that the magnetic poles and equator are not the same as the 'normal' poles and equator. Already in section 2.1 it was stated that this is indeed the case, mentioning a tilt of $47^{\circ}$ of the magnetic axis of Neptune with respect to the rotation axis. From equation (4.10) it can be seen that the magnitude decreases rapidly with increasing distance, and that the magnetic field strength over the magnetic poles is twice as strong as that over the magnetic equator. The electric charge $q$ is equal to the product of the voltage $V$ of the satellite and the capacitance $C$. The capacitance of a spherical satellite can be approximated by [Wakker, 1997b]

$$
C=1.1 \cdot 10^{-10} R_{s}
$$

with $R_{s}$ the radius of the spherical satellite. Now the formulas are known to calculate the accelerations. The values for $m_{s}$ and $R_{s}$ are again derived from the Galileo spacecraft, having $m_{s}=2,223 \mathrm{~kg}$ and $R_{s}=5.5 \mathrm{~m}$. Although Galileo is not a spherical satellite, it can be assumed to get a rough estimate of the magnitude of the disturbance acceleration. For the voltage it is assumed that it is equal to -50 V. The satellite's velocity is assumed to be equal to the local escape velocity. Now the disturbance acceleration can be calculated for various positions. As a worst case an orbit over the magnetic poles touching the planet's surface is taken. Since this is not very likely to occur also the disturbance acceleration in the magnetic equator plane at 5 planet radii is indicated in table 4.5. It can be seen from this table that the disturbance accelerations associated with electromagnetic effects are in fact very small. Even for the worst, unlikely case of a polar, surface touching orbit the accelerations are still small. This is evident from a comparison with the main surface gravitational acceleration, which is in the order of $\mathrm{m} / \mathrm{s}$. The main gravitational acceleration will decrease with distance (inversely proportional to $r^{2}$ ), but the magnetic disturbance acceleration is inversely proportional to $r^{3.5}$ (following from a combination of equations (4.9), (4.10), and (3.13)), so the relative disturbance will decrease as well. Although the electromagnetic field will probably play a role in the scientific investigation of Neptune, in the determination of the orbit it plays a negligible role. 


\subsection{Relativistic Effects}

According to the General Relativity Theory of Einstein, Newton's gravitation law is a first approximation. In this section a look will be taken at the errors made using this gravitation law, equation (3.1). It must be emphasized that the relativistic effects are not perturbing forces as discussed in the previous sections. They are caused by the small discrepancy between the used laws of physics and 'reality' as described by Einstein.

A good approximation for the relativistic movement of a particular body is given by $[$ Wakker, 1997a]

$$
\frac{\mathrm{d}^{2} u}{\mathrm{~d} \varphi^{2}}+u=\frac{\mu}{H^{2}}+3 \frac{\mu}{c^{2}} u^{2}
$$

with $\varphi$ a polar angle defined in the orbital plane and $u \equiv 1 / r$ for simplification of the differential equation. Equation (4.12) together with $H=r^{2} \dot{\varphi}$ is a system of differential equations which cannot be solved analytically. Using the method of Successive Approximations one obtains as a first order approximation [Wakker, $1997 a]$

$$
\begin{aligned}
u= & \frac{\mu}{H^{2}}[1+e \cos (\varphi-\omega)]+ \\
& 3 \frac{\mu^{3}}{c^{2} H^{4}}\left[1+\frac{1}{2} e^{2}+e \varphi \sin (\varphi-\omega)-\frac{1}{6} e^{2} \cos 2(\varphi-\omega)\right]
\end{aligned}
$$

Comparing this equation with equation (3.7) it follows that the relativistic influence is expressed by the second part between right brackets. The first two terms inside the brackets represent an amplification of $u$ by a constant value, and the third and fourth term are oscillations with increasing and constant amplitude respectively. Only the third term has an ever increasing effect, thus in the long run it will be the prevailing term for the relativistic influence. Thus equation (4.13) can be further approximated by

$$
u=\frac{\mu}{H^{2}}[1+e \cos (\varphi-\omega)+\beta e \varphi \sin (\varphi-\omega)]
$$

in which $\beta$ is defined as

$$
\beta=3 \frac{\mu^{2}}{c^{2} H^{2}}=3 \frac{\mu}{c^{2} p}
$$

From equation (4.15) it follows that the relativistic influence is larger when the central body mass increases and when the distance to the central body decreases. The largest value for $\beta$ in our solar system is for Mercury's orbit about the Sun: $\beta=8.0 \cdot 10^{-8}$. For satellites in orbit about the Earth $\beta<2.1 \cdot 10^{-9}$. Thus even for very large values of $\varphi$ it still holds that $\beta \varphi \ll 1$. For example, it takes 47990 years for Mercury to obtain $\beta \varphi=0.1$ and 1480 years to do so for a satellite rotating about the Earth at $500 \mathrm{~km}$ altitude. Thus even for long periods a small angle approximation can be used. Applying this to equation (4.14) and reintroducing $r$ gives

$$
r=\frac{H^{2} / \mu}{[1+e \cos (\varphi-\omega-\beta \varphi)]}
$$


Equation (4.16) shows that the motion can still be seen as a conical section, but now with the instantaneous position of the periapsis given by $\omega+\beta \varphi$. Thus it is a conical section with a slowly rotating major axis. This rotation was the famous verification of Einstein's Theory of General Relativity. From Newton's theory followed that the perihelion should shift 532" per century while the observations indicated 574" [Wakker, 1997a]. The relativistic effect turns out to be 43" per century; almost equal to the difference between the values just stated!

For a satellite in orbit about the Earth at $500 \mathrm{~km}$ altitude it follows that the perigee shifts 1394" per century. This value seems large compared to the value for Mercury, but it is negligible when compared to the effects of the perturbing forces discussed in the previous sections. Therefore the relativistic effect will not be taken into account for the orbital analyses.

\subsection{Implications on Trajectory}

From the discussion about perturbing forces in this chapter it became apparent that most of them only play a role (and even a minor one) when in the neighborhood of a certain planet, say in the sphere of influence. But as was shown in section 3.3 this portion of the trajectory is very short when compared to the overall flight time. And even then the gravity of the respective planet obviously prevails. Thus it can be approximated as an instantaneous velocity change based on the gravity of the planet alone, while the position does not change, after which the heliocentric orbit can continue.

On an interplanetary part, the third-body effects are the most present among the disturbances. But as can be seen from table 4.3 the solar gravitation is 2 to 8 orders of magnitude larger. Therefore, for a first order simulation and/or optimization of an interplanetary trajectory, it suffices to take only the gravity of the Sun into account. This realistic approximation enables the use of some analytical techniques for interplanetary orbits. These will be point of discussion in chapters 6 and 7. 



\section{Chapter 5}

\section{Propulsion}

In this chapter the means by which the spacecraft changes its velocity is discussed. This propulsion is needed first of all to bring the spacecraft in space, but also to move it around in space (to a certain extent). The second requirement necessitates propulsion on board the spacecraft.

There are many propulsion methods, but since this is a preliminary study for a possible near-future mission, the emphasis will lie on the readily available methods. Therefore for example the solar sail, hardly tested in practical applications, will not be a point of attention, let alone warp drives and cold fusion rockets, which require totally new principles of physics. First in section 5.1 chemical propulsion is treated, which is the only method currently applied to launchers. Then section 5.2 is devoted to electric propulsion, already used frequently for secondary propulsion, but more and more for primary propulsion as well. Launch systems will conclude this chapter in section 5.3.

\subsection{Chemical Propulsion}

From the beginning of the previous century, when Konstantin Tsiolkovsky and Robert H. Goddard first conceived the principles of rocketry, chemical propulsion has been the primary method for space transportation. It is based, like many other propulsion methods, on Newton's 3rd law: action $=$-reaction. The propulsive force exerted on the vehicle results from changes in system momentum through a highvelocity exhaust. In the case of chemical propulsion the combustion of chemical propellants, solid or liquid, at high pressure liberates large quantities of energy in a small time-span. The subsequent expansion of these high temperature products of combustion through a nozzle, converts thermal energy into kinetic energy.

\subsubsection{Basic Principles}

To discuss the properties of propulsion methods, first some basic quantities need to be introduced. First of all the thrust $T$, the force exerted by the propelling system, is found to be [Zandbergen, 2004]

$$
T=\dot{m} V_{e}
$$


with $\dot{m}$ the mass flow rate and $V_{e}$ the effective exhaust velocity, defined as a combination of the actual exhaust velocity and a term representing the pressure force on the rocket. Another important quantity is the specific impulse, the total impulse per unit propellant weight consumed, which is given by

$$
I_{s p}=\frac{I}{m_{p r} g_{0}}=\frac{\int_{0}^{t} F(t) \mathrm{d} t}{g_{0} \int_{0}^{t} \dot{m}(t) \mathrm{d} t}
$$

with $g_{0}$ the Earth standard gravitational acceleration $\left(9.80665 \mathrm{~m} / \mathrm{s}^{2}\right)$, and $m_{p r}$ the propellant mass. The effective exhaust velocity and the specific impulse are related by

$$
V_{e}=I_{s p} g_{0}
$$

Finally it can be derived that for a constant $V_{e}$ the velocity increment can be found from [Cornelisse et al., 1979]

$$
\Delta V=V_{e} \ln \left(m_{i} / m_{b}\right)+\int_{0}^{t} \frac{F_{\text {ext }}}{m} \mathrm{~d} t
$$

with $m_{i}$ and $m_{b}$ the initial and burn-out mass respectively, and $F_{\text {ext }}$ the force in the direction of motion due to external sources, such as gravity or aerodynamic drag. If it is assumed that there are no such external forces, or the burn time is short enough to neglect this term, equation (5.4) reduces to the well-known Tsiolkovsky equation

$$
\Delta V=V_{e} \ln \Lambda
$$

with $\Lambda \equiv m_{i} / m_{b}$, the ratio of initial to burn-out mass. It is quite evident from this formula that a high exhaust velocity $V_{e}$ is desirable. $\Delta V$ is typically prescribed by the mission whilst $V_{e}$ is limited by the available propulsion types and propellants. Only by increasing the mass ratio the shortfall in propellant energies can be accommodated and the mission goals be attained.

\subsubsection{Methods}

For chemical propellants there are three options: liquid, solid or hybrid, a combination of the two. The liquid variant has the highest specific impulse (300-400 s), followed by hybrid (275-300 s) and the solid variant (200-270 s) [Zandbergen, 2004]. This advantage of liquid over hybrid and solid is caused by higher combustion temperatures and lower molecular weights of the involved propellants. Furthermore liquid and hybrid engines can be throttled and restarted whereas solid motors cannot. Once the solid propellant is ignited the combustion will generally proceed until all the propellant is consumed. Therefore solid motors are not used for on board propulsion. On the other hand solid motors do have the advantage of simplicity; there is no need for separate propellant storage, pumping and feed to the injector, mixing etcetera. Because of this the solid propulsion is often used for the main engine of launchers, which requires a robust system. These launchers will be further discussed in section 5.3. The hybrid rocket motor, although having several advantages, has not been used often enough. Therefore it is not sufficiently validated to be considered for this mission. For these reasons only liquid chemical propellants are an option for the on board propulsion. Note that this is with respect to the chemical method, in section 5.2 another option will be shown. In figure 5.1 the generic layout of a liquid rocket engine is given. 


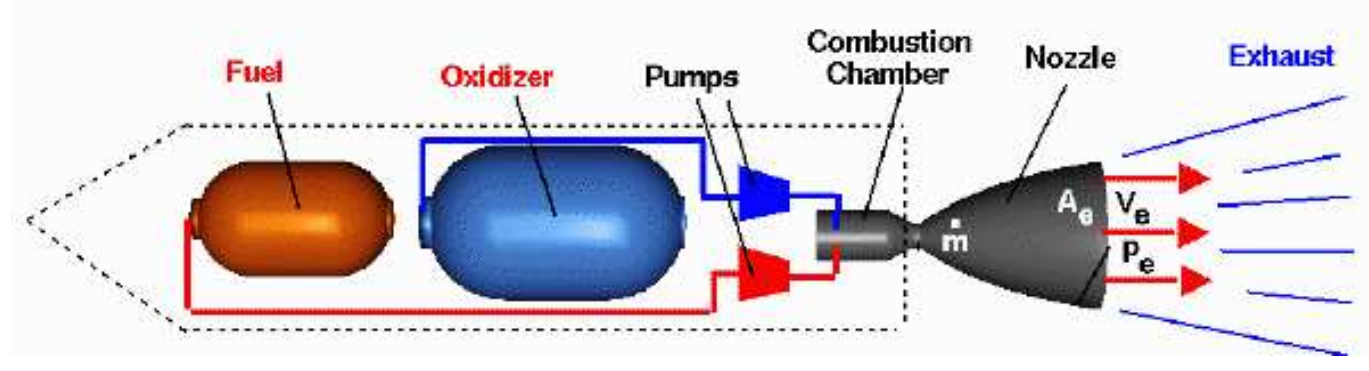

Figure 5.1 A generic layout of a liquid rocket engine [Zandbergen, 2004].

\subsubsection{Practice}

Liquid rocket engines have been the standard for on board propulsion for decades. In table 5.1 an overview of several available liquid rocket engines is given, that can be used on board spacecraft. When one is not familiar with the nomenclature used for the propellants, one is referred to the list of Acronyms. In general the combination $\mathrm{N}_{2} \mathrm{O}_{4} / \mathrm{MMH}$ is used by a motor having a dry weight of several kilograms. The general tendency of the liquid rocket engines is that the specific impulse is just above 300 seconds, while the thrust can vary from several Newtons to about $1 \mathrm{kN}$. For this thesis work, a representative specific impulse of $320 \mathrm{~s}$ is taken, and it is assumed that the thrust is high enough to enable the impulsive-shot approximation (see section 6.1).

\begin{tabular}{|c|c|c|c|c|c|}
\hline Engine & Developer & $\begin{array}{c}\text { Nominal } \\
\text { Thrust } \\
{[\mathrm{N}]}\end{array}$ & $\begin{array}{c}\text { Specific } \\
\text { Impulse } \\
{[\mathrm{s}]}\end{array}$ & Propellants & $\begin{array}{c}\text { Engine } \\
\text { Mass } \\
{[\mathrm{kg}]}\end{array}$ \\
\hline RS-21 & Rocketdyne & 1330 & 294 & $\mathrm{~N}_{2} \mathrm{O}_{4} / \mathrm{MMH}$ & 8.39 \\
\hline R-42 & Marquardt & 890 & 305 & $\mathrm{MON} / \mathrm{MMH}$ & 4.54 \\
\hline $\mathrm{R}-4 \mathrm{D}$ & Marquardt & 489 & 310 & $\mathrm{~N}_{2} \mathrm{O}_{4} / \mathrm{MMH}$ & 3.76 \\
\hline $\mathrm{DM} / \mathrm{LAE}$ & TRW & 445 & 315 & $\mathrm{~N}_{2} \mathrm{O}_{4} / \mathrm{N}_{2} \mathrm{H}_{4}$ & 4.54 \\
\hline MMBPS & TRW & 445 & 302 & $\mathrm{~N}_{2} \mathrm{O}_{4} / \mathrm{MMH}$ & 5.22 \\
\hline ADLAE & TRW & 445 & 330 & $\mathrm{~N}_{2} \mathrm{O}_{4} / \mathrm{N}_{2} \mathrm{H}_{4}$ & 4.50 \\
\hline HPLAM & TRW & 445 & 325 & $\mathrm{~N}_{2} \mathrm{O}_{4} / \mathrm{MMH}$ & 4.60 \\
\hline $\mathrm{S} 400 / 1$ & DASA & 400 & 303 & $\mathrm{MON} / \mathrm{MMH}$ & 2.8 \\
\hline RS-25 & Rocketdyne & 111 & 285 & $\mathrm{~N}_{2} \mathrm{O}_{4} / \mathrm{MMH}$ & 0.96 \\
\hline $\mathrm{R}-6 \mathrm{C}$ & Marquardt & 22.0 & 290 & $\mathrm{~N}_{2} \mathrm{O}_{4} / \mathrm{MMH}$ & 0.67 \\
\hline $\mathrm{S} 10 / 1$ & DASA & 10.0 & 287 & $\mathrm{MON} / \mathrm{MMH}$ & 0.35 \\
\hline RS-45 & Rocketdyne & 4.5 & 300 & $\mathrm{~N}_{2} \mathrm{O}_{4} / \mathrm{MMH}$ & 0.73 \\
\hline
\end{tabular}

Table 5.1 Examples of available on-board liquid rocket engines [Larson and Wertz, 1999; Zandbergen, 2004]. 


\section{$5.2 \quad$ Electric Propulsion}

Another way of propelling a spacecraft is electric propulsion. It differs from chemical propulsion in a number of ways. The most striking difference is the type of energy used, from which the names are derived. For the chemical propulsion the energy comes from the chemical energy of the propellants, but in the electric case the energy does not originate from the propellants. It is derived from a separate source instead. This source can be solar radiation or nuclear fuel. An extension of the basic principles discussed in section 5.1.1 is given to clarify this difference.

\subsubsection{Basic Principles}

The power related with the jet of gas expelled is equal to the time derivative of the involved kinetic energy:

$$
P_{j}=\frac{1}{2} \dot{m} V_{e}^{2}
$$

with $P_{j}$ the jet power. This is related to the output power of the energy source as follows

$$
P_{P}=P_{j} / \eta
$$

with $\eta$ the thrust efficiency. With equation (5.1) the last two equations can be combined to

$$
P_{P}=\frac{1}{2} T V_{e} / \eta
$$

Assuming a constant exhaust velocity, the thrust $T$ is linearly related to the output power $P_{P}$.

\subsubsection{Methods}

Also with electric propulsion the propellants are expelled at high speeds through the exhaust, forcing an acceleration of the spacecraft in the opposite direction. This even higher velocity, when compared to chemical propulsion, can be attained in three different ways: electrothermally, in which the enthalpy of the propellant is increased and converted into directed kinetic energy via a nozzle; electrodynamically, in which crossed electric and magnetic fields induce a Lorentz force in a plasma; and electrostatically, in which charged particles are accelerated directly in an electric field [Zandbergen, 2004].

The electrothermal thruster comes in two main types: the resistojet, in which the propellant is heated via a resistance element, and the arcjet, when the propellant is heated by passing it through an arc discharge. These electrothermal thrusters have a modest specific impulse of $300-800 \mathrm{~s}$ when compared to other means of electric propulsion. Therefore they are not suitable for forming the primary propulsion on high-energy missions. Since they have a relatively low mass, high value for thrust over power, and flight experience they are a serious option for the secondary propulsion.

The electrodynamic thrusters can be subdivided in the following types: MagnetoPlasmaDynamic (MPD) thrusters, Pulsed Inductive Thrusters (PITs) and 
Pulsed Plasma Thrusters (PPTs) [Larson and Wertz, 1999]. Of these only the last one has some flight experience. The others still impose development risks. And above all, the performance of the PPT in terms of specific impulse is lower than that of the following type of electric propulsion.

The electrostatic thrusters have the highest performance. The specific impulse of Hall Effect Thrusters (HETs) can go up to $2000 \mathrm{~s}$, but the ion thrusters can even go past $3000 \mathrm{~s}$ [Zandbergen, 2004]. On high-energy missions this is highly preferable, and therefore electrostatic ion engines are superior when it comes to interplanetary trajectories.

\subsubsection{Practice}

Electric propulsion has been used for primary propulsion on board spacecraft only two times. Firstly on NASA's Deep Space 1 spacecraft, launched in 1998, the NSTAR ion engine was used for primary propulsion. Secondly ESA's SMART-1 spacecraft, which orbited the Moon, carried a PPS-1350 Hall Effect Thruster (see figure 5.2). Both were used as technology demonstrators. The most relevant specifications of these systems can be found in table 5.2.

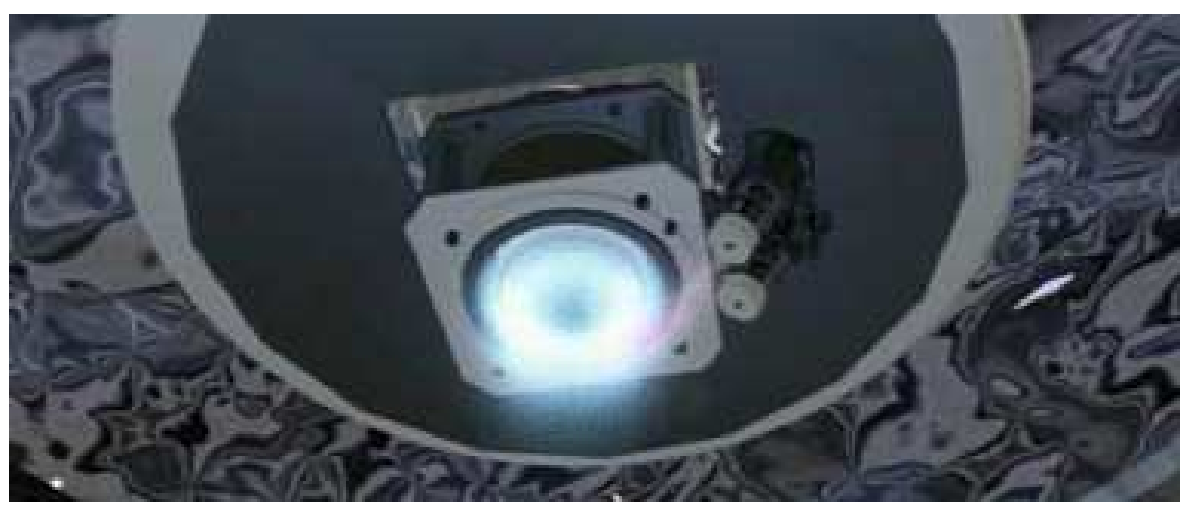

Figure 5.2 SMART-1's Hall effect thruster ignited [ESA, 2006c].

\begin{tabular}{|l|c|c|}
\hline & Deep Space 1 (NSTAR) & SMART-1 (PPS-1350) \\
\hline Thrust $[\mathrm{mN}]$ & 92 & 68 \\
Specific Impulse $[\mathrm{s}]$ & 3100 & 1640 \\
Power Input $[\mathrm{kW}]$ & 2.3 & 1.19 \\
\hline
\end{tabular}

Table 5.2 Primary electric propulsion in practice: Deep Space 1 [NASA/JPL, 2002] and SMART-1 [ESA, 2006c].

Clearly, the performance of the HET used on the SMART-1 mission is less than that of the Deep Space 1 ion engine. Partly for this reason the ESA missions BepiColombo to Mercury and the Solar Orbiter will use new ion thrusters. With these new ion engines, thrust levels of up to $250 \mathrm{mN}$ (resulting in acceleration levels in the order of tenths of $\mathrm{mm} / \mathrm{s}^{2}$ for masses in the order of $1000 \mathrm{~kg}$ ) and specific impulses of $3500 \mathrm{~s}$ are attainable [ESA-SCI, 2000]. In ESA-SCI [2000] also the power requirements can be found: 1 to $5 \mathrm{~kW}$ per engine, depending on the thrust level. These engines can be throttled, enabling the application of optimal thrust magnitudes. Although not completely developed and validated yet, these new generations of ion engines will be applied in this graduation project for the 
following reason. If the conceptual mission and spacecraft design would rely solely on off-the-shelf technology, it would result in conservative estimates that may not be attractive or truly representative of the mission as it actually will be designed and built. Hence, in this thesis work a constant specific impulse of $3500 \mathrm{~s}$ is taken, with a maximum thrust of $250 \mathrm{mN}$, obtained at a power level of $5 \mathrm{~kW}$.

These kind of power levels are obtainable with currently available solar arrays at a distance of $1 \mathrm{AU}$ [ESA-SCI, 2000]. The lowest possible power level at which the engine still functions is $1 \mathrm{~kW}$, at which $50 \mathrm{mN}$ is delivered (due to the assumed linear relationship between thrust and power, see equation (5.8)). At a particular distance from the Sun, this power level cannot be attained anymore. The incoming solar flux will decrease proportionally to the distance squared according to equation (4.8). This is somewhat offset by increasing efficiency through the reduction in the temperature of the solar cells. Taking this effect into account the produced power decreases by a factor $\left(r / r_{E}\right)^{1.5}$ [Fortescue et al., 2003].

\subsection{Launch Systems}

To select a set of possible launchers one can first take a look at the launchers used for similar missions. For Galileo the Space Shuttle was used. This reusable launch system is not foreseen to have a long future remaining. It is more likely that it will almost exclusively be used for constructing the remainder of the International Space Station (ISS), after which it will be disposed. Therefore the Space Shuttle will not be considered as an option. Cassini-Huygens was launched by a Titan rocket. Since the whole Titan family is out of service since October 19, 2005, due to high operation costs, this is simply not an option.

Having discarded the launchers of similar missions, other launchers should be studied. It is not the intention to make a full overview of all the available launchers around the world. Instead a selection of 4 rocket families is made: Ariane, Soyuz, Proton, and Atlas.

A perhaps logical choice would be the European Ariane launcher, since this will be an ESA mission. The newest member of the Ariane family is the Ariane 5; the older Ariane rockets are out of service. Ariane 5 has a modest experience in putting spacecraft in an Earth escape trajectory: only the Rosetta comet hunter had this honor. Having a wet mass of $3065 \mathrm{~kg}$ it was given a hyperbolic escape velocity of $3545 \mathrm{~m} / \mathrm{s}$ [Perez, 2004]. The maximum payload capacity is $3190 \mathrm{~kg}$, because in the performance figures also booster adapter weight and a performance margin are taken into account. The Rosetta vehicle was launched by an Ariane 5 Generic. The more powerful version Ariane $5 \mathrm{ECA}$ is able to give the mentioned hyperbolic escape velocity to a total of $4300 \mathrm{~kg}$ [Perez, 2004]. These performance data are indicated in figure 5.3, together with the performance of other launchers, like the Soyuz. As is the custom, the performance is expressed in $C_{3}$-values, of which the square root renders the velocity at infinity.

The Soyuz family has an impressive history which started with launching the first satellite (Sputnik, 1957) and the first man (Yuri Gagarin, 1961). Since 1996 it is commercially exploited by Starsem, a partnership with $50 \%$ European and $50 \%$ Russian ownership. It has an incredible experience of 820 flights up to and including the year 2000 [Starsem, 2001]. Predominantly these were 'terrestrial' 


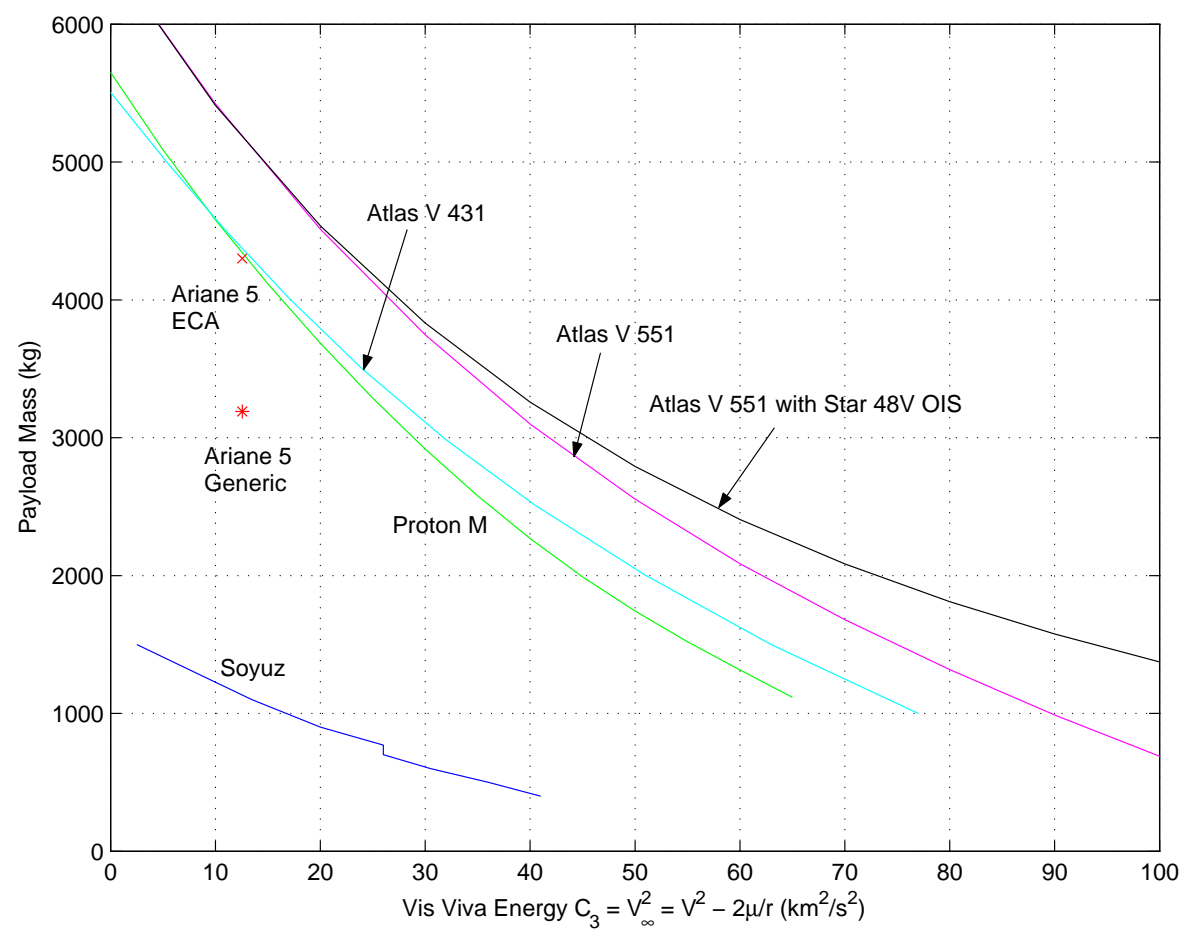

Figure 5.3 Earth escape performance of various launch vehicles [see text for sources].

flights; it is the workhorse of the International Space Station, for example. Its Earth escape performance is limited, as can be seen in figure 5.3, even though the Fregat upper stage is included [Starsem, 2001]. The costs are relatively low, as indicated in table 5.3.

\begin{tabular}{|l|c|}
\hline Launch Vehicle & Unit Cost $[\mathrm{FY} 00 \$ \mathrm{M}]$ \\
\hline Ariane V & $155-186$ \\
Soyuz & $31-52$ \\
Proton M & $93-101$ \\
Atlas V 551 & $90^{*}$ \\
\hline
\end{tabular}

Table 5.3 Cost of various launch vehicles [Wertz, 2001]. *Estimated

The Proton rocket has also been operational for a long time, being operational from 1970 onwards. As of October 2004 it has carried out more than 287 launches [Laursen, 2004]. It is commercially exploited since 1995 by International Launch Services (ILS), a joint Russian-American venture. The Proton M in combination with the Breeze $\mathrm{M}$ upper stage is able to inject a spacecraft into an Earth escape trajectory. Its performance and cost are again depicted in figure 5.3 and table 5.3, respectively. It has a significantly better performance when compared to the Ariane 5 Generic and the Soyuz rocket.

Last, but certainly not least, is the Atlas rocket. It has flown 588 times since its first flight in 1957 [Sponnick and Jensen, 2004]. It also belongs to the launch fleet of ILS. To meet the increasingly demanding payload requirements, the Atlas V family has been created. There exist two series: the 400 and 500 series, with a payload fairing diameter of 4 and 5 meters, respectively. The most powerful in these series are the 431 and 551, having 3 and 5 Solid Rocket Boosters (SRBs), respectively. The 1 indicates that it uses a Centaur upper stage. The Atlas V 
551 was responsible for the launch of the New Horizons spacecraft. This Pluto encountering spacecraft was given a record hyperbolic excess velocity of almost $12 \mathrm{~km} / \mathrm{s}[N A S A, 2006 a]$. For these high energy requirements an extra upper stage can be included, for example the Star 48V Orbit Insertion Stage (OIS). The performance data of these 3 options, Atlas V 431 and Atlas V 551 with and without the Star 48V OIS, are indicated in figure 5.3 [Sponnick and Jensen, 2004]. It shows that the upgraded Atlas V 551 can indeed enable high-energy missions.

Having discussed these four launchers (see figure 5.4) a small trade-off can be made. Although being the only strictly European launcher, the Ariane 5 rocket will not be the chosen launcher due to its very modest experience with Earth escape flights and its high costs. The Soyuz on the other hand can provide a low-cost launch, but its performance is significantly lower than that of the other ones. Two options remain: the Proton and Atlas rocket. For both the costs are medium, and they each have a high performance. But on both aspects the Atlas outperforms the Proton launcher slightly. Therefore a choice for the Atlas seems logical, but experience shows that a spacecraft should be designed to be compatible with several launch systems. In that way launch probability is enhanced, while at the same time it provides some leverage in negotiating launch cost. Since both the Atlas and the Proton are exploited by the same company ILS, the second point does unfortunately not hold. But the launch probability is certainly enlarged. For computations in this thesis work the most powerful launcher is taken: the Atlas V 551 with the Star $48 \mathrm{~V}$ OIS. For this purpose the relation between payload mass $m_{\text {payl }}$ and $C_{3}$-value is stated:

$$
m_{\text {payl }}=-1.44 \cdot 10^{-3} \cdot C_{3}^{3}+0.627 \cdot C_{3}^{2}-98.7 \cdot C_{3}+6.36 \cdot 10^{3}
$$

It has been obtained by fitting a polynomial in a least-squares sense to the data supplied by [Sponnick and Jensen, 2004].

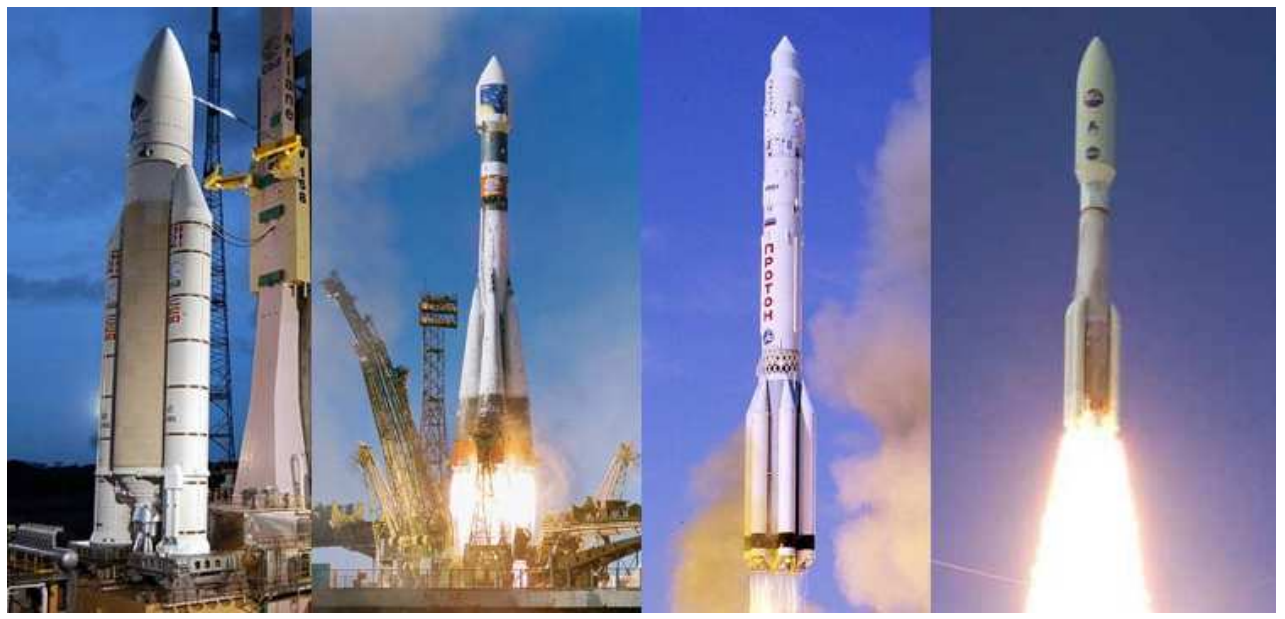

Figure 5.4 Various launch vehicles in the early phases of launch. from left to right: Ariane 5G launching Rosetta, Soyuz with Fregat upper stage, Proton launching Zvezda ISS module, and Atlas V 551 launching New Horizons. 
Chapter 6

\section{High-Thrust Trajectories}

In this chapter it will be shown how the high-thrust trajectories will be computed and optimized. In section 6.1 the general method of patched conics will be explained, which will be followed by the generation of heliocentric conics in section 6.2 using Lambert targeting. The theory about swing-bys from section 3.4 will be extended to powered swing-bys in section 6.3 and to aerogravity assists in section 6.4 .

\subsection{Patched Conics}

The principles described in chapter 3 will now be used for interplanetary trajectories. How these can be quite accurately approximated by means of patching conics (a succession of Keplerian orbits) is the subject of this section.

The idea is to approximate the interplanetary trajectory by three consecutive conic sections: first a geocentric hyperbola, followed by a heliocentric section, and at last a hyperbola again, but now with respect to the target planet. This approximation is possible due to two already described effects. As can be seen in the third column of table 3.1 the sphere of influence is quite small with respect to the interplanetary distances; in case of the Earth it is less than $1 \%$ and for Neptune less than $2 \%$ of their respective distances to the Sun. Furthermore it was shown at the end of section 3.2 that at a distance of about 400,000 $\mathrm{km}$ the local velocity is almost equal to the hyperbolic excess velocity. Then at a distance of about $10^{6}$ $\mathrm{km}$ (approximate radius of the sphere of influence) it can safely be assumed that the local velocity is equal to this $V_{\infty}$. The same holds for the other planets in our solar system.

Furthermore it is assumed that the planets move in circular orbits around the Sun and that all planetary orbits lie in the ecliptic plane, i.e. the geometric plane containing the mean orbit of the Earth around the Sun. In section 6.1.1 the effect of this approximation will be discussed. This assumption is not necessary for applying patched conics, but it plays an enabling role in the example at the end of this section. Another assumption is that the interplanetary flight starts from a parking orbit around the Earth, and chemical propulsion will be used for leaving the Earth and capturing at the target planet. With this high-thrust propulsion (see section 5.1) it can be assumed that the spacecraft experiences an instantaneous 
velocity change, leaving the spacecraft's position unaltered. But when must this thrust be applied? For that purpose the energy change per unit mass is considered

$$
\Delta \mathcal{E}=\frac{1}{2}\left(V_{0}^{2}-V_{c_{0}}^{2}\right)=\frac{1}{2}\left(\Delta V_{0}\right)^{2}+\mathbf{V}_{c_{0}} \cdot \Delta \mathbf{V}_{0}
$$

with $\mathbf{V}_{c_{0}}$ the spacecraft's velocity in the circular parking orbit just before the impulsive shot and $\mathbf{V}_{0}$ just after. From this relation it can be seen that it is most advantageous if the thrust is applied tangentially to the parking orbit, and at a location where the orbital velocity is a maximum. The last statement is of no importance for the circular parking orbit but does imply that the impulse at the target planet should be applied at the periapsis of the hyperbolic leg.

In addition to the direction of the thrust and point of application, the required velocity increase is of utmost importance. It can be calculated using equations (3.10) and (3.15)

$$
\Delta V_{0}=V_{0}-V_{c_{0}}=\sqrt{\frac{2 \mu_{E}}{r_{0}}+V_{\infty_{E}}^{2}}-\sqrt{\frac{\mu_{E}}{r_{0}}}
$$

with $r_{0}$ the radius of the parking orbit and the perigee distance of the hyperbolic escape trajectory. In a similar way follows for the capturing delta- $\mathrm{V}$ at the target planet

$$
\Delta V_{3}=V_{3}-V_{c_{3}}=\sqrt{\frac{2 \mu_{t}}{r_{3}}+V_{\infty_{t}}^{2}}-V_{p a r_{3}}
$$

with $r_{3}$ the periapsis distance of the hyperbolic trajectory around the planet and $V_{\text {par }_{3}}$ the periapsis velocity in the parking orbit just after deceleration, which should obviously be lower than the local escape velocity. It should be noted that the parking orbit is not necessarily circular. For the periapsis distances values can be assumed, but for the hyperbolic excess velocities a more in-depth discussion is needed first.

Looking at figure 6.1 the geometry of the heliocentric section has some variables involved. It can be shown that the most energy-efficient trajectory is the one with $\gamma_{1}=\gamma_{2}=0 \quad$ [e.g., Cornelisse et al., 1979]. This results in half an ellipse and is called a Hohmann trajectory. For this to occur the hyperbolic excess velocity should be aligned with Earth's orbital velocity around the Sun. Therefore

$$
V_{\infty_{E}}=V_{1}-V_{E}=\sqrt{\frac{\mu_{S}}{a_{E}}}\left(\sqrt{2-\frac{a_{E}}{a_{i t}}}-1\right)
$$

where $\mu_{S}$ is the solar gravitation parameter, $a_{i t}$ is the semi-major axis of the interplanetary Hohmann trajectory, and $a_{E}$ is the semi-major axis of the orbit of the Earth. In a similar way the hyperbolic excess velocity with respect to the target planet can be found:

$$
V_{\infty_{t}}=V_{t}-V_{2}=\sqrt{\frac{\mu_{S}}{a_{t}}}\left(1-\sqrt{2-\frac{a_{t}}{a_{i t}}}\right)
$$

with $a_{t}$ the semi-major axis of the orbit of the target planet. Now the required delta- $\mathrm{V}$ for a Hohmann trajectory to any planet can be calculated. Neptune will be taken as an example, since this is the target in this thesis. The values needed 


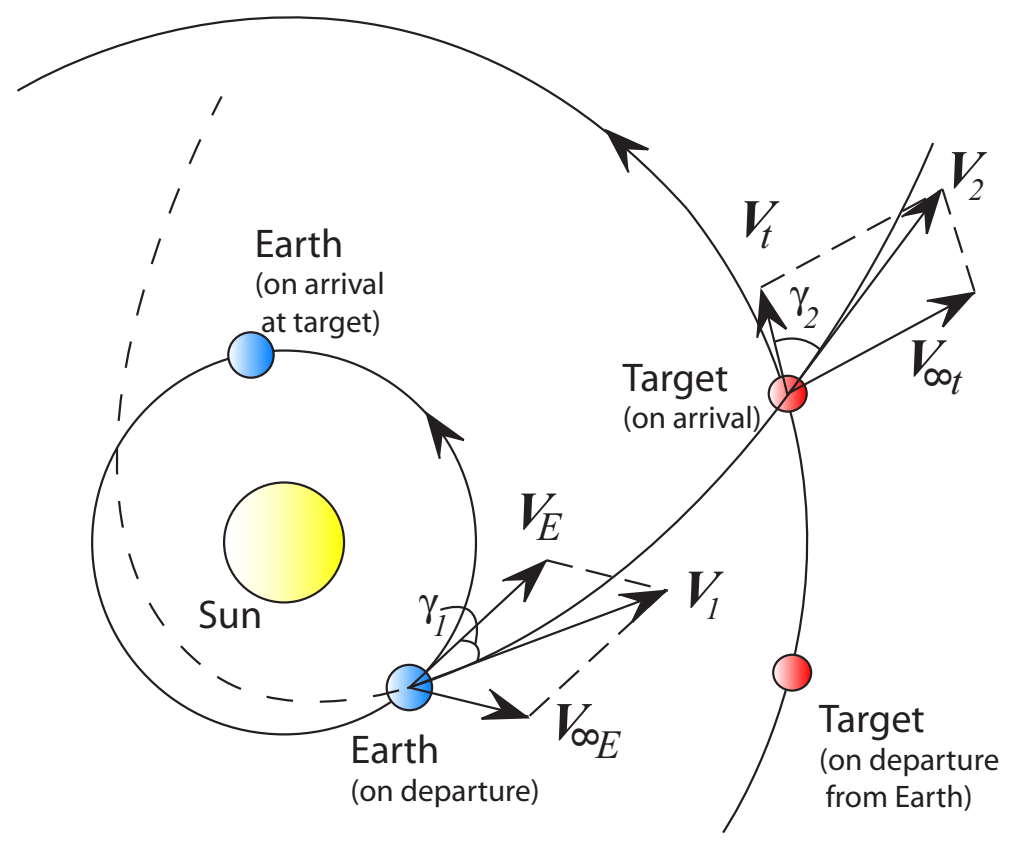

Figure 6.1 Geometry of two-dimensional interplanetary trajectory (not to scale) (adapted from [Cornelisse et al., 1979]).

in equations (6.4) and (6.5) are all known, except for $a_{i t}$, but this is equal to $\frac{1}{2}\left(a_{N}+a_{E}\right)$. Substituting those values results in

$$
V_{\infty_{E}}=11.65 \mathrm{~km} / \mathrm{s} \quad ; \quad V_{\infty_{N}}=4.05 \mathrm{~km} / \mathrm{s}
$$

Then assuming that the parking orbit around the Earth is at an altitude of 185 $\mathrm{km}$, and the capture orbit around Neptune is at 1.1 times Neptune's radius, the delta-V's can be found, using equations (6.2) and (6.3):

$$
\Delta V_{0}=8.25 \mathrm{~km} / \mathrm{s} \quad ; \quad \Delta V_{3}=6.98 \mathrm{~km} / \mathrm{s}
$$

These delta-V's are appreciably large, and are hard, if not impossible, to be obtained by using today's launchers, as was shown in section 5.3. Therefore one has to fall back to other ideas. The flight time follows from equation (3.12), and is found to be 30.6 years. This is quite a long time, which hints in the direction of faster trajectories, requiring even larger delta-V's, or smarter solutions. Therefore the gravity assist discussed in section 3.4 is of utmost importance and will be more extensively dealt with in sections 6.3 and 6.4 .

\subsubsection{Three-Dimensional Trajectories}

In the previous section it was assumed that the planetary orbits are circular and lie in the same plane. Although the eccentricities and inclinations of the planets are quite small (0.0097 and $1.774^{\circ}$ respectively in the case of Neptune), it can introduce significant errors. In [Cornelisse et al., 1979] it is shown that for flights to Venus and Mars the required delta-V can increase up to about 5\%, although it can also be lower. To show how these values are obtained goes into too much detail. The purpose of this section is just to indicate that for the orbital analysis to be performed it can not be neglected that interplanetary trajectories are threedimensional. 
For this reason a three-dimensional ephemeris model is used. At the chair of Astrodynamics and Satellite Systems, Delft University of Technology, NASA's Jet Propulsion Laboratory (JPL) DE200 series is used. The JPL Solar System Ephemeris specifies the past, present and future positions of the Sun, Moon, and nine planets in three-dimensional space. The DE200 series is in the J2000 coordinate system and gives the three-dimensional position and velocity components of the planets. The J2000 equatorial coordinate system is defined by the mean orientation of Earth's equator and the equinox at January 1, 2000, 12:00 Terrestrial Time (a time scale to serve as the independent argument for geocentric ephemerides). For interplanetary flights normally the ecliptic plane is used as a reference. The ecliptic plane contains the mean orbit of the Earth around the Sun. The transformation from the equatorial to the ecliptic reference frame simply comprises of a rotation of $23.44^{\circ}$ about the $x$-axis (pointing to the vernal equinox). The ecliptic reference frame is more practical than the equatorial reference frame, since the orbits of the planets around the Sun approximately lie in this ecliptic plane.

\subsection{Lambert Targeting}

To be able to design an interplanetary trajectory, transfer orbits between planets need to be determined. In section 6.1 a minimum-energy transfer, the Hohmann transfer orbit, was discussed. Then the resulting trajectory is simply an ellipse with the one planet at perihelion and the other at aphelion. It was shown that it gives a flight time of about 30.6 years, obviously much too long for a viable mission. This creates the need of finding orbits and corresponding velocities for different geometries. This can be done using so-called Lambert targeting, which is described by Gooding [1990] as follows:

An unperturbed orbit, about a given inverse-square law center of force, say

$F$, is to be found connecting two given points, $P_{1}$ and $P_{2}$, with a specified

flight time $\Delta t=t_{2}-t_{1}$.

The geometry is shown in figure 6.2. In the practical case at hand the Sun is at the center of force, the focus $\mathrm{F}$ of the orbit. $P_{1}$ and $P_{2}$ describe the positions of two planets at times $t_{1}$ and $t_{2}$ respectively, from which the time of flight follows easily. The assumption is that it is a two-body problem (the Sun and the satellite) as described in section 3.2, so a conical section will come out of the Lambert Targeting procedure.

It should be clear that at least two solutions exist; one could go clockwise or anti-clockwise. For both directions of motion an orbit can be found which satisfies the time requirement. This duality can be taken away by specifying the direction of motion in advance. Since all the planets orbit around the Sun in an anti-clockwise direction, it is wise to choose the same direction for the motion of the satellite; choosing the clockwise direction would require an enormous amount of energy to compensate for the planet's velocity.

Another complication comes into play when considering one or more full revolutions between the two encounters. It can be shown that for a specific number of full revolutions ( 1 or higher) two unique solutions exist for a predefined direction of 


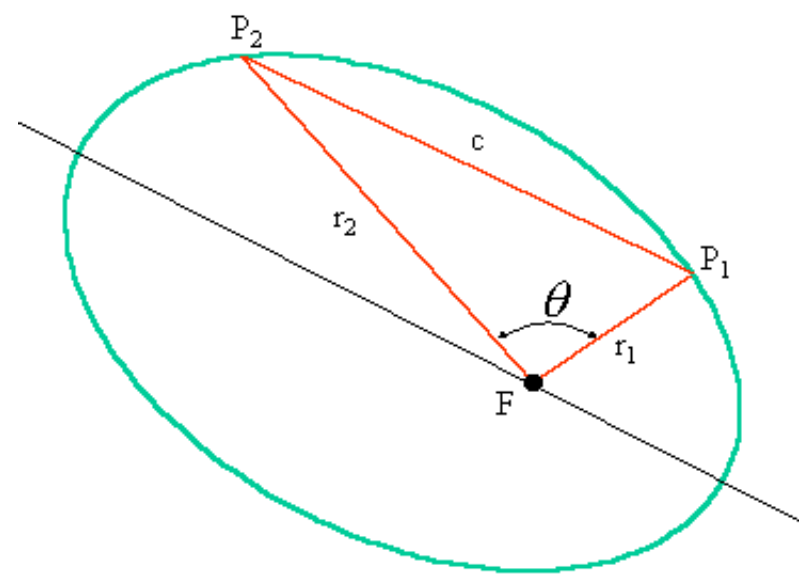

Figure 6.2 Transfer orbit geometry of Lambert problem [Battin and Vaughan, 1983].

motion, provided that the time of flight $\Delta t$ is long enough. Hence, as $\Delta t$ increases for a given triangle $F P_{1} P_{2}$ and specified direction of motion, the number of solutions $N$ is an increasing odd integer, apart from being even (instantaneously) at each value of $\Delta t$ at which two new solutions emerge. An example of two different paths having the same time of flight is shown in figure 6.3. This complication is partly compensated by not restricting the transfer angle $\theta$ to be between 0 and $2 \pi$, but by regarding it as an angle of unrestricted positive magnitude defined by the geometry of the path and not just by that of the triangle. Then, if $\theta<2 \pi, N=1$, meaning that only one solution exists for less than one full revolution. However, if $\theta>2 \pi$, the number of full revolutions before the final part to the target planet is one or more, being $\theta / 2 \pi$ rounded off downwards. In the case that the specified time of flight is simply not long enough to complete the specified number of full revolutions, no solution exists, so $N=0$. Otherwise 2 solutions exist as stated before, unless the time is equal to the $\Delta t$ at which these two solutions emerge. Thus, $N=0,1$ or 2 if $\theta>2 \pi$.

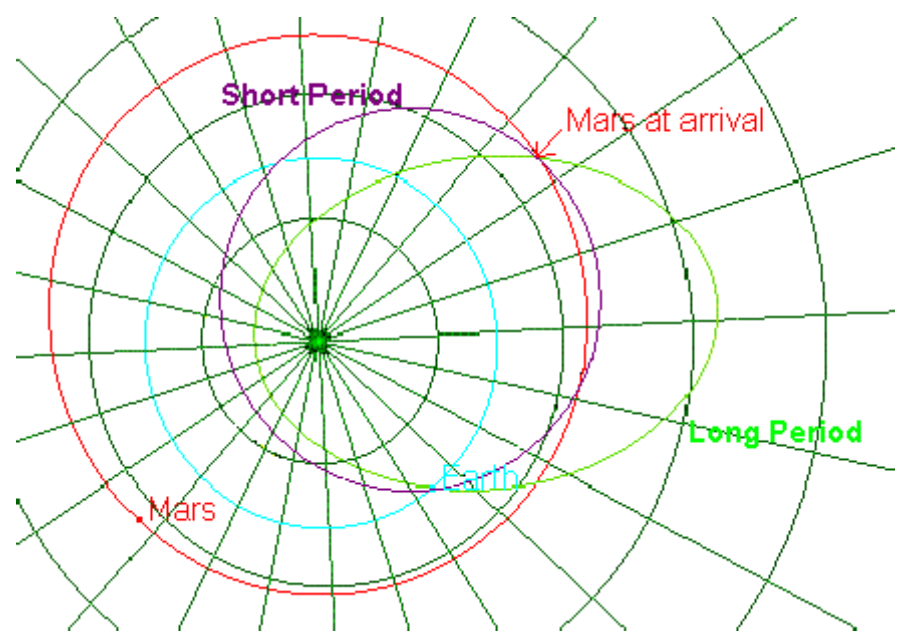

Figure 6.3 Two solutions for an Earth-Mars transfer using more than one full revolution [JAQAR, 2005]. After orbiting the Sun once and returning to the original position of the Earth, the trajectory is continued until the second intersection with the orbit of Mars. At this point both solutions/trajectories encounter Mars after the same time of flight (being 660 days). 
The algorithm required for solving the Lambert problem can take many forms, all having their disadvantages and advantages. It started with the classical method devised by Gauss, attractive because of its simplicity. Unfortunately it has two major flaws: the method is singular for a transfer angle of $180^{\circ}$ and the convergence rate is extremely slow when that angle is not very small [Battin and Vaughan, 1983]. Therefore Battin and Vaughan [1983] have devised an algorithm that takes away these flaws. Then again, Gooding [1990] did this as well, albeit in a different way, also providing the possibility of implementing multiple revolutions. It also speaks for Gooding [1990] that the full computer algorithm is stated in his paper. For these two reasons the algorithm by Gooding [1990] will be applied for this graduation thesis, and will be summarized in the rest of this section. For a full derivation one is referred to [Gooding, 1990] and the references mentioned in that paper.

A formula relating the time of flight and the orbital elements is [Wakker, $1997 a]$ :

$$
\Delta t=\sqrt{\frac{a^{3}}{\mu}}\left(E_{2}-E_{1}-e\left(\sin E_{2}-\sin E_{1}\right)\right)
$$

with $a$ the semi-major axis, $\mu$ the gravitational parameter of the primary body (the Sun in this case) and $E$ the eccentric anomaly (the angle between the direction of periapsis and the current position of an object on its orbit, projected onto the ellipse's circumscribing circle perpendicularly to the major axis, measured at the center of the ellipse). This equation can be rewritten by various substitutions, to be better suited for iterations. First of all, the primary variables $\Delta t$ and $a$ are non-dimensionalized to the following variables:

$$
\begin{aligned}
T & =\sqrt{\frac{8 \mu}{s^{3}}} \Delta t \\
x^{2} & =1-\frac{s}{2 a}
\end{aligned}
$$

with s the semi-perimeter: $s=\frac{r_{1}+r_{2}+c}{2}$. Equation (6.6) can now be expressed in the dimensionless variables $T$ and $x$ :

$$
T=\frac{2(x-q z-d / y)}{E_{l a m}}
$$

This equation again introduces some new parameters. First of all, $q$ follows directly from the problem geometry:

$$
q=\frac{\sqrt{r_{1} r_{2}}}{s} \cos (\theta / 2)
$$

Now $E_{l a m}, y$, and $z$ can be computed:

$$
\begin{aligned}
E_{\text {lam }} & =x^{2}-1 \\
y & =\sqrt{\left|E_{\text {lam }}\right|} \\
z & =\sqrt{1-q^{2}+q^{2} x^{2}}
\end{aligned}
$$

The computation of $d$ takes more effort. First $f$ and $g$ are calculated:

$$
\begin{aligned}
& f=y(z-q) \\
& g=x z-q E_{l a m}
\end{aligned}
$$


Then if $E_{l a m}<0$ (and thus an elliptic orbit) $d=\arctan (f / g)$. If $E_{l a m}>0$ (and thus an elliptic orbit) $d=\ln (f+g)$. Every quantity for the iteration formula (6.9) has been defined. For the iteration the Halley method is used by Gooding [1990]. It uses the following algorithm to ensure fast convergence from the starting value for $x$ to the value of $x$ for which the desired $T$ results [Burden and Faires, 2001]:

$$
x_{n+1}=x_{n}-\frac{f\left(x_{n}\right) f^{\prime}\left(x_{n}\right)}{2 f^{\prime}\left[\left(x_{n}\right)\right]^{2}-f\left(x_{n}\right) f^{\prime \prime}\left(x_{n}\right)}
$$

where $f\left(x_{n}\right)=T-2\left(x_{n}-q_{n} z_{n}-d_{n} / y_{n}\right) / E_{l a m_{n}}$, as it follows from equation (6.9), and with $f^{\prime}$ and $f^{\prime \prime}$ the first and second derivative, respectively, of $f$ with respect to $x$. To ensure even faster convergence appropriate starters have been developed by Gooding [1990]. In figure 6.4 a plot of $T$ versus $x$ can be seen, for various values of $q$ and $m$, indicating the number of full revolutions. This plot can be used for determining the starters. As an example the behavior of $T$ for $m=0$ is taken. It should be clear that it has an important dependency on the sign of $x$ (again see figure 6.4). Therefore, for $x>0$ and $x<0$ different starters are devised. First the sign of $x$ is determined by evaluating $T$ (equation (6.9)) for $x=0$, denoted $T_{0}$. Then, the sign of $x$ is equal to the sign of $T_{0}-T$, as it follows from figure 6.4. The starting value for $x, x_{0}$, can be found using one of the following formulas, depending on the sign of $x$ [Gooding, 1990]:

$$
\begin{aligned}
& x>0 \Rightarrow x_{0}=\frac{T_{0}\left(T_{0}-T\right)}{4 T} \\
& x<0 \Rightarrow x_{0}=-\frac{T-T_{0}}{T-T_{0}+4}
\end{aligned}
$$

The starting formula for $x<0$ is found to work much less well than the other one and a patch is needed. For the explanation of this patch one is referred to [Gooding, 1990]. Also starters for multiple revolutions can be found in that paper. However, in this thesis work, multiple revolutions are ignored in the case of Lambert targeting, so $m$ is assumed to be 0 .

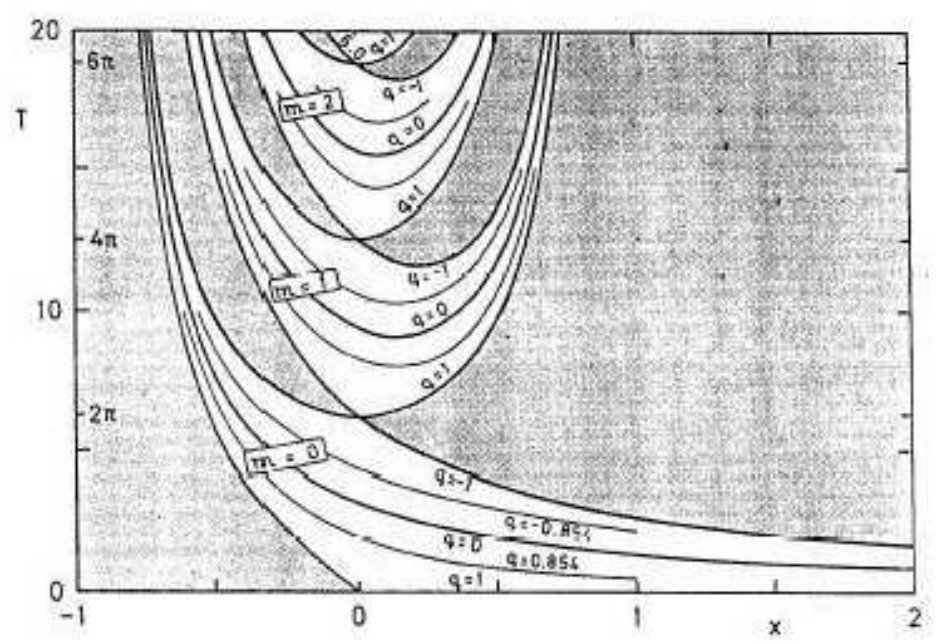

Figure 6.4 Plot of $T$ versus $x$ for selected values of $q$ and $m$ [Gooding, 1990].

Using these starters and the Halley iteration method, convergence is achieved within three iteration steps. By then $\varepsilon<10^{-13}$, with $\varepsilon$ defined as the smaller 
of the relative error in $x$ and the relative residual in $T$ (the rationale is that the accuracy in a numerical solution of the general equation $f(X)=Y$ should always be assessed in terms of the numerically smaller of the relative error in $X$ and the relative residual in $Y$; assessment in terms of just the former could amount to a demand for the impossible) [Gooding, 1990].

After the described iterations the solution for $x$ is known, and the semi-major axis can be found using equation (6.8). However, for the problem at hand the velocities are more interesting. Gooding [1990] has gathered and improved the formulas to find these velocities, which resulted in:

$$
\begin{aligned}
& V_{r, 1}=\gamma[(q z-x)-\rho(q z+x)] / r_{1} \\
& V_{r, 2}=-\gamma[(q z-x)+\rho(q z+x)] / r_{2} \\
& V_{c, 1}=\gamma \sigma(z+q x) / r_{1} \\
& V_{c, 2}=\gamma \sigma(z+q x) / r_{2}
\end{aligned}
$$

where

$$
\begin{aligned}
& \gamma=\sqrt{\mu s / 2} \\
& \rho=\left(r_{1}-r_{2}\right) / c \\
& \sigma=2 \sqrt{r_{1} r_{2} / c^{2}} \sin (\theta / 2)
\end{aligned}
$$

These radial $\left(V_{r}\right)$ and circumferential $\left(V_{c}\right)$ velocity components lie in the plane spanned by $F P_{1}$ (or $\mathbf{r}_{1}$ ) and $F P_{2}$ (or $\mathbf{r}_{2}$ ), so the motion is now completely defined.

These computations can now be performed for each heliocentric leg in between planets. But there is certainly no guarantee that these conics can be patched by using the gravity of the respective planet alone. Therefore in the next section it is explained how this is effectuated by the application of an engine burn.

\subsection{Powered Gravity Assist}

With the theory described in section 3.4 it is straightforward how to compute the parameters of an un-powered swing-by. This is only possible if the arrival velocity at infinity matches the departure velocity at infinity: $V_{\infty_{i}}=V_{\infty_{f}}$, and the required bending angle is smaller than the maximum achievable bending angle from equation (3.25), with $r_{p, \text { limit }}$ substituted for $r_{p}$. In general this is not the case, especially the desired agreement between the two velocities. When the velocities at infinity are different one has to resort to a powered swing-by. The maximum achievable bending angle, taking into account different velocities, is:

$$
\alpha_{\max }=\frac{\alpha_{i}}{2}+\frac{\alpha_{f}}{2}=\arcsin \frac{1}{1+\frac{r_{p, l i m i t} V_{\infty_{i}}^{2}}{\mu}}+\arcsin \frac{1}{1+\frac{r_{p, l i m i t} V_{\infty_{f}}^{2}}{\mu}}
$$

The geometry of such an asymmetrical swing-by can be seen in figure 6.5. If the required bending angle is larger than the maximum bending angle, $r_{p}$ is simply equated to $r_{p, l i m i t}$. How to deal with the lack of bending is discussed later on. If the required bending angle is smaller than the maximum, the value for $r_{p}$ that 


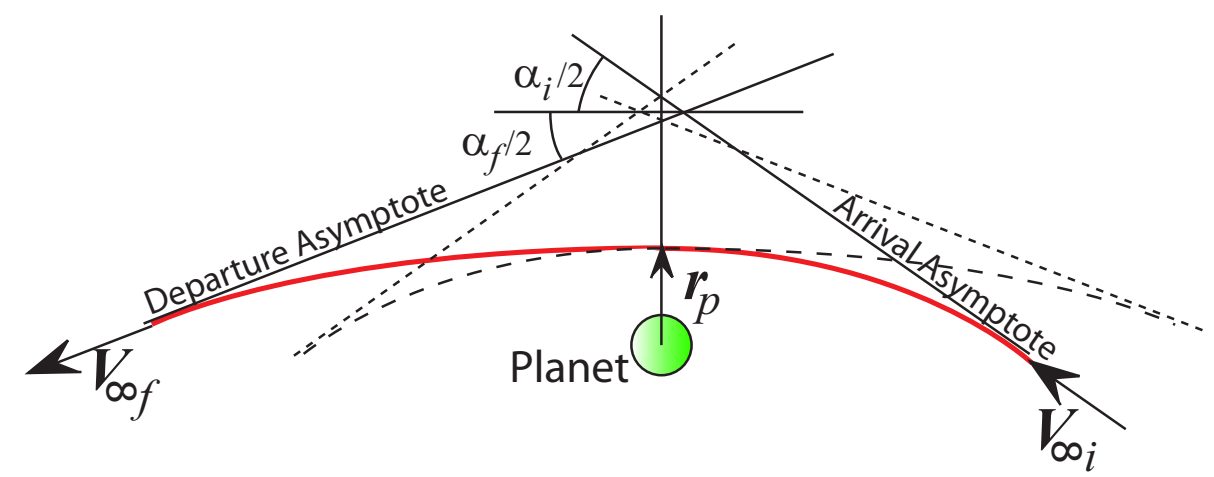

Figure 6.5 Geometry of a powered swing-by, with $V_{\infty_{f}}>V_{\infty_{i}}$.

results in this angle needs to be found. It is the solution of the following equation, being quite similar to equation (6.26):

$$
\alpha=\frac{\alpha_{i}}{2}+\frac{\alpha_{f}}{2}=\arcsin \frac{1}{1+\frac{r_{p} V_{\infty_{i}}^{2}}{\mu}}+\arcsin \frac{1}{1+\frac{r_{p} V_{\infty_{f}}^{2}}{\mu}}
$$

This equation cannot be solved directly and an iterative method should be applied. To that end another variable is used, the eccentricity $e$. The eccentricity of the incoming hyperbolic leg differs from that of the outgoing one, but are related as follows:

$$
r_{p}=a_{i}\left(1-e_{i}\right)=a_{f}\left(1-e_{f}\right)
$$

The semi-major axis follows easily from equation (3.14). Using equation (3.25), equation (6.27) can now be rewritten as:

$$
\alpha=\arcsin \frac{1}{e_{i}}+\arcsin \frac{1}{e_{f}}=\arcsin \frac{1}{e_{i}}+\arcsin \frac{1}{1-\frac{a_{i}}{a_{f}}\left(1-e_{i}\right)}
$$

Now iteration can take place. In this case the Newton-Raphson method is used:

$$
e_{i_{n+1}}=e_{i_{n}}-\frac{f\left(e_{i_{n}}\right)}{f^{\prime}\left(e_{i_{n}}\right)}
$$

where

$$
f\left(e_{i_{n}}\right)=\arcsin \frac{1}{e_{i_{n}}}+\arcsin \frac{1}{1-\frac{a_{i}}{a_{f}}\left(1-e_{i_{n}}\right)}-\alpha
$$

and $f^{\prime}\left(e_{i_{n}}\right)$ is simply the derivative of $f\left(e_{i_{n}}\right)$ with respect to $e_{i_{n}}$. It concerns a hyperbolic orbit, $e>1$, and therefore the starting value $e_{i_{0}}$ can be set, somewhat arbitrary, to 1.01. After the eccentricities of both the incoming and outgoing hyperbolic leg are computed using the described iteration scheme, the velocities in periapsis can be computed using equation (3.14):

$$
\begin{aligned}
V_{p_{i}}^{2} & =V_{\infty_{i}}^{2}\left(\frac{e_{i}+1}{e_{i}-1}\right) \\
V_{p_{f}}^{2} & =V_{\infty_{f}}^{2}\left(\frac{e_{f}+1}{e_{f}-1}\right)
\end{aligned}
$$


The required velocity change can now be determined as the absolute difference between the two:

$$
\Delta V_{1}=\left|V_{p_{f}}-V_{p_{i}}\right|
$$

Applying this delta- $\mathrm{V}$ at periapsis enables the connection of the arrival and departure hyperbolic legs. In the case that the required bending angle is smaller than the maximum obtainable angle, the swing-by can be successfully performed without further complications. Otherwise, one still needs to rotate the velocity vector a bit more over an angle $\Delta \alpha=\alpha-\alpha_{\max }$, see figure 6.6. The magnitude of $\Delta V_{2}$ is now easily determined:

$$
\Delta V_{2}=2 V_{\infty} \sin (\Delta \alpha / 2)
$$

No subscript $i$ or $f$ has been indicated, since it holds irrespective of where this velocity change is applied. Obviously, it is most efficient to apply it where the velocity is lowest, at arrival or at departure. The combination of $\Delta V_{1}$ and $\Delta V_{2}$ results in the total $\Delta V$ to perform a powered swing-by. To conclude, in a good case $\Delta V_{2}$ is not necessary, and in an ideal case the arrival and departure velocities match and the swing-by does not need a velocity change at all.

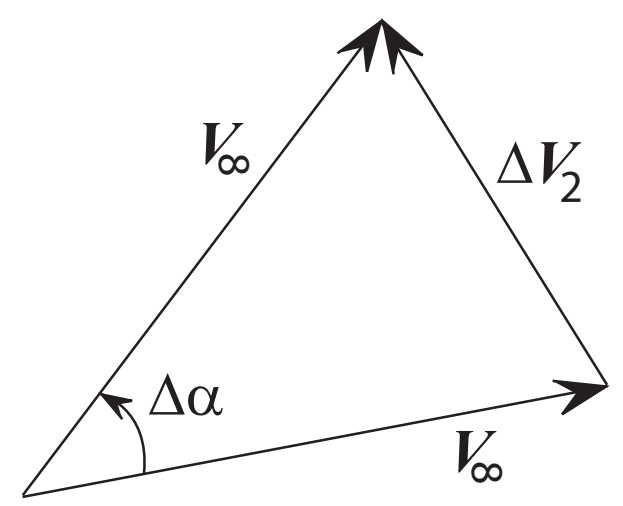

Figure 6.6 Geometry for the determination of $\Delta V_{2}$.

\subsection{The Aerogravity Assist}

In section 3.4 it was shown that the gravity assist can be very useful in interplanetary flight for supplying 'free' energy. But there were a few restrictions, a limited deflection angle $\alpha$ being one of them (see equation (3.25)). This restriction can be taken away by applying a so-called aerogravity assist. This assist takes advantage of the aerodynamic forces on a spacecraft when it moves through a planet's atmosphere. The atmosphere keeps the spacecraft for an extended period of time in close proximity to the planet, enlarging the deflection angle. In this way the final hyperbolic excess velocity $\mathbf{V}_{\infty_{f}}$ can be more aligned with the planet's heliocentric velocity $\mathbf{V}_{t}$. Although the final hyperbolic excess velocity will be smaller than the initial one due to drag, by aligning the velocity vectors the final heliocentric speed can be increased. This is in short how it works in words, it will now be dealt with more in-depth and mathematically (similar to [Anderson Jr. et al., 1991]).

The first part until entering the atmosphere is similar to the gravity assist, and therefore the same formulas as in section 3.4 can be applied. Once in the 


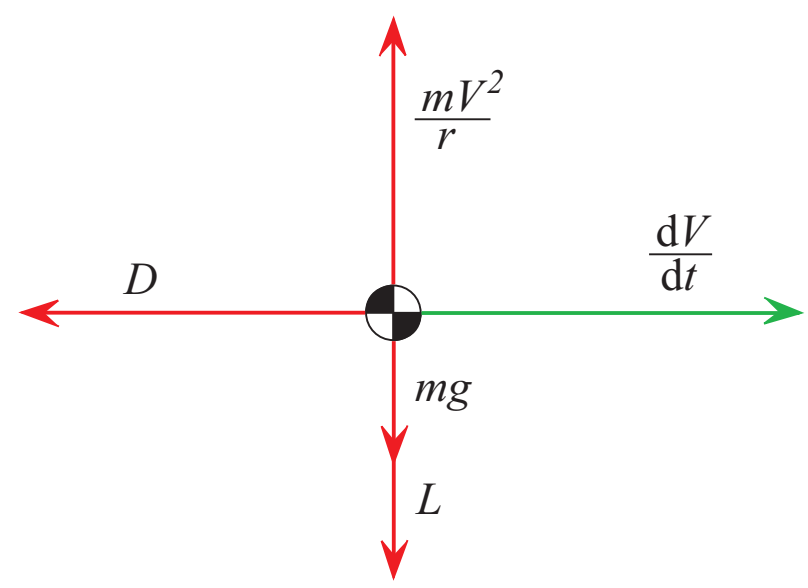

Figure 6.7 The forces (red) and acceleration (green) on the center of gravity of a spacecraft during an aerogravity assist. Note that, using this convention, the acceleration is negative.

atmosphere at its periapsis distance $r_{p}$ the aerodynamic forces come into play. These can be seen in figure 6.7, amongst other forces. Applying Newton's second law in direction of flight gives

$$
m_{s} \frac{d V}{d t}=-D
$$

with $D$ the drag force. Since it is most convenient to characterize the vehicle in terms of the lift-to-drag ratio $L / D$ this can be rewritten as

$$
d V=-\frac{L}{(L / D) m_{s}} d t
$$

The time flown through the atmosphere can be related to the aerodynamic bending angle $\alpha_{a b}$ by considering the arc length of the trajectory through the atmosphere $V \mathrm{~d} t=r_{p} \mathrm{~d} \alpha_{a b}$. This angle $\alpha_{a b}$ can be found in figure 6.8, in which the geometry of an aerogravity assist is depicted. In this equation it is already assumed that the flight is at a constant altitude. The lift is used for that purpose. Since it is desired to keep the spacecraft 'down', instead of flying away from the planet by the centrifugal forces, the lift must be pointed towards the planet (the spacecraft will fly upside down). The constant altitude implies that there is a vertical force equilibrium between the downward lift $L$, gravity $m g$, and the outward centrifugal force (see figure 6.7):

$$
L+\frac{m_{s} \mu}{r_{p}^{2}}=\frac{m_{s} V^{2}}{r_{p}}
$$

so that equation (6.37) can be written as

$$
\mathrm{dV}=-(L / D)^{-1}\left(V-\frac{\mu}{r_{p} V}\right) \mathrm{d} \alpha_{a b}
$$

Integrating, assuming that $L / D$ is constant, gives for the aerodynamic bending angle

$$
\alpha_{a b}=\frac{1}{2}\left(\frac{L}{D}\right) \ln \left[\frac{V_{p_{i}}^{2}-\mu / r_{p}}{V_{p_{f}}^{2}-\mu / r_{p}}\right]
$$




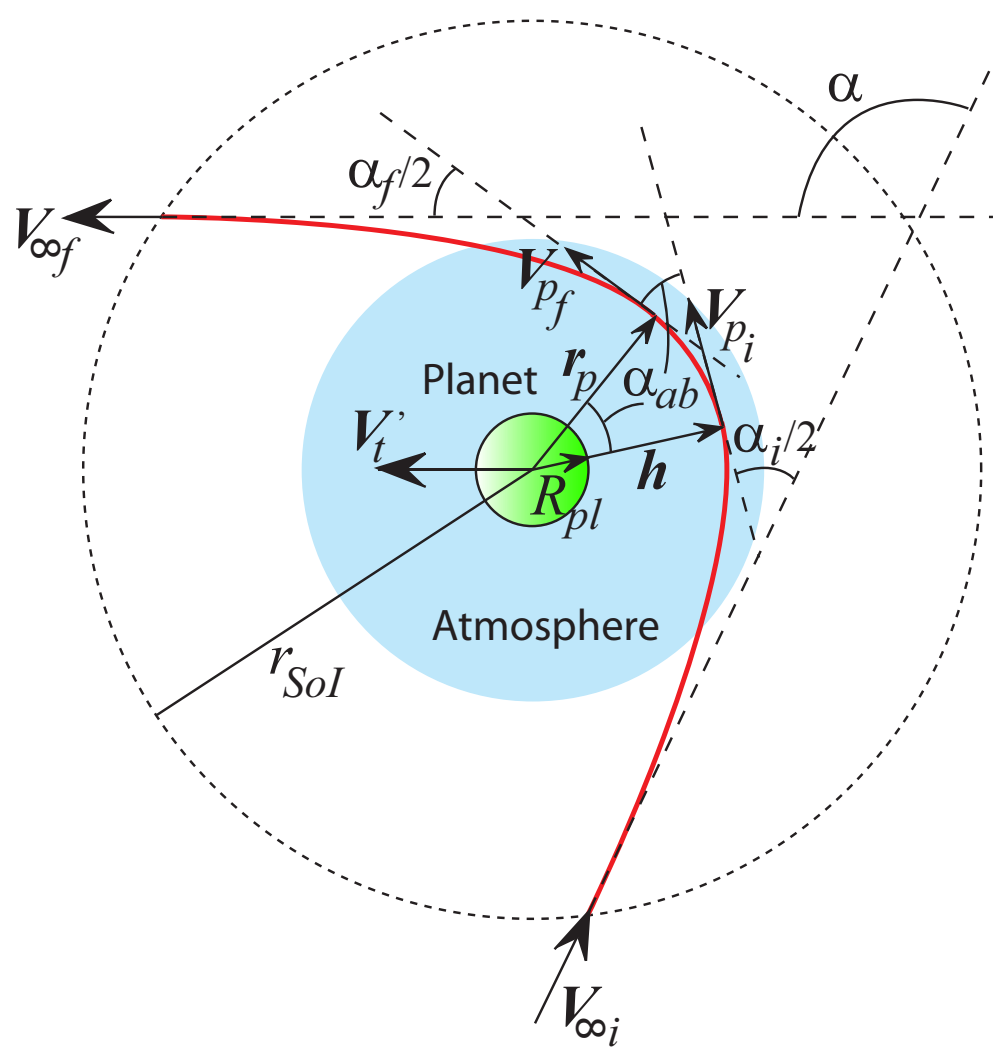

Figure 6.8 Geometry of an aerogravity assist (not to scale).

Isolating $V_{p_{f}}$ gives

$$
V_{p_{f}}=\sqrt{e^{-2 \alpha_{a b} /(L / D)}\left(V_{p_{i}}^{2}-\mu / r_{p}\right)+\mu / r_{p}}
$$

Then the total turn angle follows from

$$
\alpha=\alpha_{i} / 2+\alpha_{a b}+\alpha_{f} / 2
$$

The increase in the total turn angle with respect to the pure gravity assist is now quite obvious. The angle increases for increasing lift-to-drag ratio $L / D$ as can be found from equation (6.40). However, the options are limited: a high $L / D$ cannot be obtained with conventional spacecraft. These are not designed for atmospheric flight. Therefore some other type of spacecraft is necessary for these kind of missions. A hypersonic waverider is thought to be the most eligible candidate according to [Anderson Jr. et al., 1991] and [Lewis and McRonald, 1992]. This craft is designed such that the shock wave remains attached to the leading edge, giving the appearance that the vehicle is riding on top of the shock wave. The high lift is generated by the high pressure behind the shock wave, under the spacecraft. According to several studies, for example [Lewis and McRonald, 1992], $L / D$ can be as high as 15 , but can easily drop down to 5 , depending on the exact circumstances. In this thesis work, an $L / D$ of 10 is taken as a baseline.

Another important aerodynamic parameter is the wingload $\frac{m_{s}}{C_{L} A}$. It is hidden in equation (6.38), which can also be written as

$$
\frac{1}{2} \rho V_{p}^{2} C_{L} A+\frac{m_{s} \mu}{r_{p}^{2}}=\frac{m_{s} V_{p}^{2}}{r_{p}}
$$


with $\rho$ the atmospheric density of a particular planet, $C_{L}$ the lift coefficient, and $A$ a reference area. This equation should hold, since it is used in the derivation of equation (6.41). The density can be isolated from this equation to give

$$
\rho=\left[1-\left(\frac{V_{c}}{V_{p}}\right)^{2}\right] \frac{2}{r_{p}}\left(\frac{m_{s}}{C_{L} A}\right)
$$

with $V_{c}$ the circular velocity at radius $r_{p}\left(=\sqrt{\mu / r_{p}}\right)$. This density is not a constant for a given atmosphere, but depends primarily on height. A model which is relatively simple on the one hand and gives a good correspondence with reality on the other is the exponential atmosphere, of the form [NASA/GSFC, 2006]

$$
\rho(h)=\rho_{s} \cdot e^{-h / H}
$$

with $h$ the height of the spacecraft position, $\rho_{s}$ the surface density, and $H$ the scaling height, i.e. the height interval in which the atmospheric density changes by a factor of $e=2.7183$. The height can of course be found as follows: $h=r_{p}-R_{p l}$. The values for the parameters $\rho_{s}$ and $H$ are taken from [NASA/GSFC, 2006] for every planet with an atmosphere and these values can be found in table 6.1. Since the gas giants do not have a clear solid surface, it is noted that the stated densities (just like the radii and diameters) are for levels corresponding to a pressure of 1 bar. Equations (6.44) and (6.45) can be combined to form

$$
\rho_{s} \cdot e^{-h / H}=\left[1-\left(\frac{\frac{\mu}{r_{p}}}{\frac{2 \mu}{r_{p}}+V_{\infty_{i}}^{2}}\right)\right] \frac{2}{r_{p}}\left(\frac{m}{C_{L} A}\right)
$$

This equation depends on the planet at which the AGA is performed (this defines $\rho_{s}, H, R_{p l}$, and $\mu$ ), the incoming hyperbolic leg (defining $V_{\infty_{i}}$ ), the wingload and the height. Usually the first two are fixed when calculating a gravity assist. For the wingload a representative value of $50 \mathrm{~kg} / \mathrm{m}^{2}$ is taken [McRonald and Randolph, 1992]. Therefore the height is prescribed by equation (6.46) whereby vertical equilibrium is attained. This equation cannot be solved analytically since the height occurs both in and outside exponents (remember that $r_{p}$ also depends on the height). Therefore an iterative procedure is used to solve this equation, namely the Newton-Raphson method (see equation (6.30)). As a numerical illustration the heights and associated densities are noted in table 6.1 for initial velocities at infinity of both 0 and $50 \mathrm{~km} / \mathrm{s}$. Although a velocity of $0 \mathrm{~km} / \mathrm{s}$ is not very realistic, it forms a theoretical lower bound. It can be seen that, although the heights do not differ much with $V_{\infty_{i}}$, the height becomes lower as the velocity at infinity increases. This is to be expected, since the centrifugal force will be larger at a higher velocity. To counteract this the lift should increase as well, which can be accomplished by flying at lower altitudes where the density is higher. On top of that, the gravity will also increase (be it a little) at lower altitudes.

Another numerical illustration can be given, indicating the repercussions of taking a different height than the one prescribed by the vertical equilibrium. For example, take a flight through the atmosphere of Venus at a velocity equal to the escape velocity (the lower bound), both at the prescribed height of $253.1 \mathrm{~km}$ and at a height of $80 \mathrm{~km}$. For these conditions the associated accelerations are indicated in table 6.2. In the case of the prescribed height, the gravity and the lift force combined can exactly counteract the centrifugal force, as expected. On the other hand, at an altitude of $80 \mathrm{~km}$, the atmospheric density becomes relatively 


\begin{tabular}{|l|c|c|c|c|c|c|}
\hline & & & \multicolumn{2}{|c|}{$V_{\infty_{i}}=0 \mathrm{~km} / \mathrm{s}$} & \multicolumn{2}{|c|}{$V_{\infty_{i}}=50 \mathrm{~km} / \mathrm{s}$} \\
Planet & $\rho_{s}\left[\mathrm{~kg} / \mathrm{m}^{3}\right]$ & $H[\mathrm{~km}]$ & $\rho\left[\mathrm{kg} / \mathrm{m}^{3}\right]$ & $h[\mathrm{~km}]$ & $\rho\left[\mathrm{kg} / \mathrm{m}^{3}\right]$ & $h[\mathrm{~km}]$ \\
\hline Venus & 65 & 15.9 & $7.93 \cdot 10^{-6}$ & 253.1 & $1.56 \cdot 10^{-5}$ & 242.4 \\
Earth & 1.217 & 8.5 & $7.72 \cdot 10^{-6}$ & 101.7 & $1.51 \cdot 10^{-5}$ & 96.0 \\
Mars & 0.020 & 11.1 & $1.44 \cdot 10^{-5}$ & 80.3 & $2.87 \cdot 10^{-5}$ & 72.7 \\
Jupiter & 0.16 & 27 & $6.96 \cdot 10^{-7}$ & 333.3 & $9.85 \cdot 10^{-7}$ & 323.9 \\
Saturn & 0.19 & 59.5 & $8.20 \cdot 10^{-7}$ & 735.0 & $1.37 \cdot 10^{-6}$ & 704.6 \\
Uranus & 0.42 & 27.7 & $1.93 \cdot 10^{-6}$ & 340.4 & $3.57 \cdot 10^{-6}$ & 323.4 \\
Neptune & 0.45 & 19.7 & $2.00 \cdot 10^{-6}$ & 242.8 & $3.64 \cdot 10^{-6}$ & 231.0 \\
\hline
\end{tabular}

Table 6.1 The importance of the height in obtaining vertical equilibrium during an aerogravity assist [NASA/GSFC, 2006].

high $\left(\rho=0.42 \mathrm{~kg} / \mathrm{m}^{3}\right)$, resulting in an extreme acceleration, namely $450 \mathrm{~km} / \mathrm{s}^{2}$. This indicates that the acceleration levels due to the aerodynamic forces become extremely high when taking a too low altitude.

\begin{tabular}{|l|c|c|c|}
\hline & \multicolumn{3}{|c|}{ Acceleration $\left[\mathrm{m} / \mathrm{s}^{2}\right]$ due to } \\
& Gravity & Lift Force & Centrifugal Force \\
\hline$h=253.1 \mathrm{~km}$ & 8.18 & 8.17 & 16.35 \\
$h=80 \mathrm{~km}$ & 8.64 & $4.50 \cdot 10^{5}$ & 17.28 \\
\hline
\end{tabular}

Table 6.2 Acceleration during an aerogravity assist at different heights at Venus. The velocity is equal to the local escape velocity.

The discussed aerogravity assist can also be used to slow down, just as the pure gravity assist can. If the purpose is to put the spacecraft in orbit around the planet, it is called an aerocapture. For this aerocapture in principle the same formulas apply, the only difference being that the final velocity will be lower than the escape velocity. It is the idea to apply this method to capture at Neptune. Therefore it will be optimized for the atmosphere of this planet. This implies that the spacecraft does not have an optimal configuration for other atmospheres, since those can be significantly different. Although this has suboptimal effects, the aerogravity assist during flybys at other planets than Neptune can still have better results than pure gravity assists. However, it should be noted that these kind of maneuvers have never been performed before in an actual mission. This results in a significant development risk. But if conceptual mission and spacecraft design would rely solely on readily available techniques, it would result in conservative estimates that may not be attractive or truly representative of the mission as it actually will be designed and built. The aerogravity assist will therefore be seriously considered. 


\section{Low-Thrust Trajectories}

The trajectories discussed in chapter 6 assumed a high-thrust propulsion to enable an instantaneous velocity change, the impulsive shot. With low-thrust propulsion this is not attainable. Thus, for example, entering a Hohmann trajectory using only low-thrust propulsion is not possible. The principles behind and options for low-thrust propulsion were point of discussion in chapter 5. In this chapter a general discussion about the associated trajectories is started in section 7.1, followed by the method chosen for modeling these trajectories analytically: the exponential sinusoid. Its shape and dynamics are treated in section 7.2, the associated algorithm in section 7.3, and finally a verification of its implementation in section 7.4.

\subsection{General Characteristics}

First a look will be taken at the launch phase. As a direct consequence of the low thrust, electric propulsion cannot be used to launch a vehicle from the Earth's surface. But if an electrically propelled stage is launched into a parking orbit around the Earth, even a minute thrust will force the vehicle to recede gradually from the parking orbit, resulting in spiral trajectories [Breakwell and Rauch, 1966]. These spiral trajectories are very time consuming since they require tens or even hundreds of revolutions to escape. On top of that, current rocket launchers are perfectly capable of putting spacecraft in a direct escape trajectory. The onboard low-thrust propulsion will therefore more effectively be applied during the interplanetary trajectory.

Proceeding to this interplanetary part, the question arises what the optimal thrust direction is. Studies reveal that this optimal direction varies with time in quite a complex way [Cornelisse et al., 1979]. However, they indicate as well that a thrust tangential to the orbit is very close to the optimal direction. This can be shown by considering the equation of motion as in equation (3.3), but now with the thrust acceleration a added:

$$
\ddot{\mathbf{r}}+\frac{\mu}{r^{3}} \mathbf{r}=\mathbf{a}
$$

Scalar multiplication of equation (7.1) by $\mathbf{V}$ gives the instantaneous rate of change of the specific total energy

$$
\frac{\mathrm{d} \mathcal{E}}{\mathrm{d} t}=\mathbf{V} \cdot \mathbf{a}
$$


From equation (7.2) it can be seen that the instantaneous rate of change of energy is a maximum if the thrust is acting tangential to the trajectory. But when constraints act on the trajectory, this is not so obvious anymore. For example, it is possible that during its interplanetary tour the spacecraft has to make a flyby at a specific planet. In that case tangential thrusting does not ensure that it will actually make a close encounter in an optimal way. It could also be the case that a thrust anti-parallel to the velocity is preferable, or at least with a component in the negative velocity direction: in this way an encounter with Venus for a swing-by for example is possible with a smaller launch delta- $\mathrm{V}$ than the one necessary for a Hohmann transfer. This can be seen in figure 7.1.

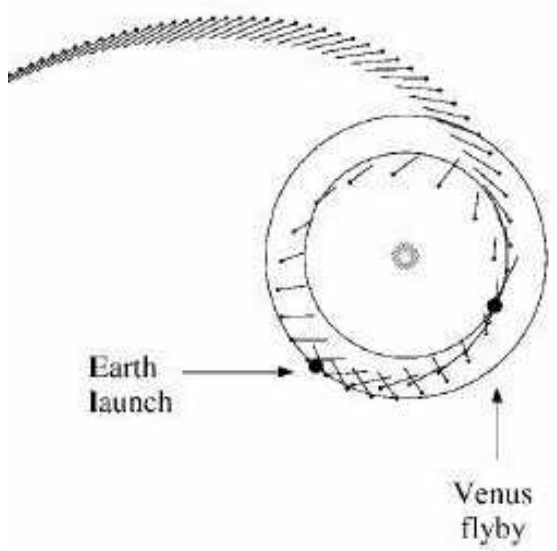

Figure 7.1 Optimized low-thrust interplanetary trajectory indicating the thrust direction [McConaghy et al., 2003]. The small dots indicate the point at which the thrust is applied, and the attached lines indicate the direction.

The actual optimal thrust direction, in addition to its magnitude, depends on many variables, like planet configuration, maximum thrust, mass etc [McConaghy et al., 2003]. It is a complicated problem which cannot be tackled directly by taking all the possible variations into account [Petropoulos and Longuski, 2004]. Therefore some simplifying assumptions are made enabling the use of a shapebased approach, implementing not the shape of a conic (ballistic trajectories) but the so-called exponential sinusoid. This method will be discussed in the remainder of this chapter. The approach is largely based on the papers by Petropoulos and Longuski [2000, 2004]. It should be noted that this comprises in fact a preliminary search of the immense search space, which does not ensure that the best possible trajectory is found. Normally this method would be followed by an optimization which constrains the possible trajectories less severely (making less assumptions), but due to practical time constraints this was not possible. Nonetheless, a solution provided by the exponential sinusoids gives a realistic trajectory, which could display the attractiveness of using high-efficiency low-thrust engines.

\subsection{Shape and Dynamics}

While applying a shape-based approach, the orbit shape is determined first, similar to Lambert targeting. From the shape then follows the required thrust profile. Some assumptions are the same as for the 'high-thrust trajectories', described earlier: multi-body effects are neglected, and gravity assists are considered as 
instantaneous velocity changes. Aerogravity assists are not an option, since the required power comes from large solar panels, which are certainly not suitable for a high-velocity flight through an atmosphere. In addition, the assumption is made that the spacecraft trajectory between gravity assists is roughly planar. Petropoulos et al. [1999] present the examination of various planar shapes which could be used as a baseline for low-thrust trajectories. It is desirable that a few shape parameters can describe a variety of realistic trajectories, thus reducing the number of computations needed to determine an arc, given certain conditions. Also the required thrust profile should not give excessive or extremely fluctuating results. Based on these requirements the exponential sinusoid was selected, given here in polar coordinates $r$ and $\theta$ :

$$
r=k_{0} e^{k_{1} \sin \left(k_{2} \theta+\phi\right)}
$$

with $k_{0}$ the scale parameter, $k_{1}$ the dynamic range parameter, $k_{2}$ the winding parameter, and $\phi$ a phase angle. These parameters are constants for a particular shape. To demonstrate the effect of $k_{2}$, two examples of an exponential sinusoid are shown in figure 7.2. The smaller this winding parameter, the more revolutions about the central body from periapsis to apoapsis. From equation (7.3) the number of revolutions $n_{p 2 a}$ can easily be deduced:

$$
n_{p 2 a}=\frac{1}{2 k_{2}}
$$

The ratio of apoapsis radius to periapsis radius is governed by $k_{1}$, and can be derived from equation (7.3) as well:

$$
\frac{r_{a}}{r_{p}}=e^{2 k_{1}}
$$
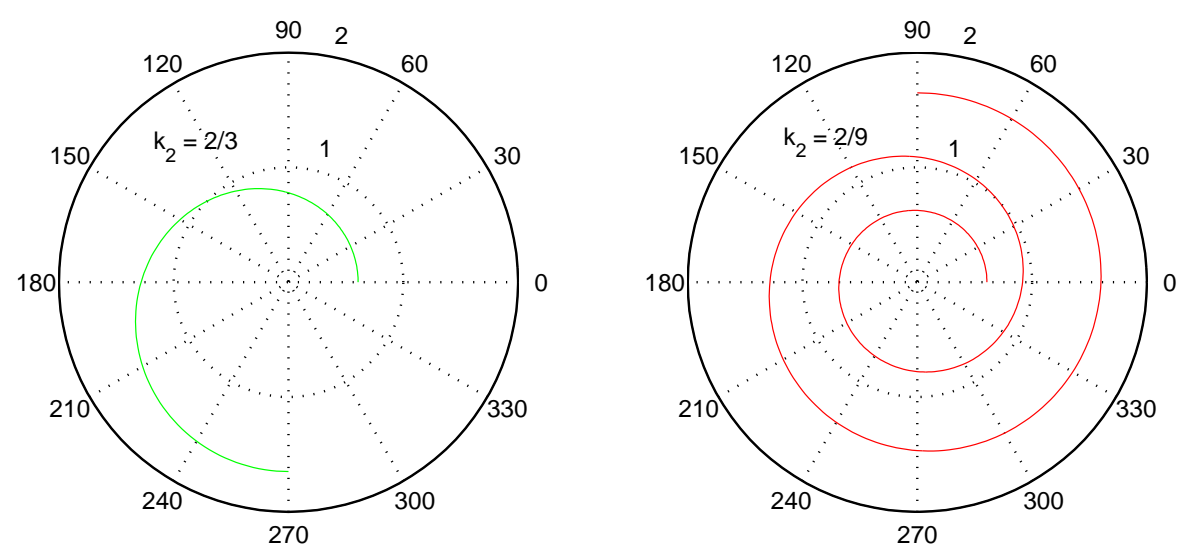

Figure 7.2 Sample exponential sinusoids, shown from periapsis to apoapsis, for $k_{0}=1, k_{1}=0.5$, and $\phi=-\pi / 2$.

Having introduced the exponential sinusoid, the associated velocities and accelerations can be discussed. First the assumption is made that the thrust is (anti-)parallel to the velocity vector. This is because it is the simplest analytic case, has tolerable thrust-acceleration levels and attractive velocity profiles, and together with the shape assumption it defines a unique thrust profile. This thrust profile can be found by looking at the two-body equations of motion in polar coordinates [Cornelisse et al., 1979]:

$$
\ddot{r}-r \dot{\theta}^{2}+\frac{\mu}{r^{2}}=a_{T} \sin \alpha
$$




$$
2 \dot{r} \dot{\theta}+r \ddot{\theta}=a_{T} \cos \alpha
$$

with $a_{T}$ the thrust acceleration, and $\alpha$ the angle of the thrust vector with the local horizon (being perpendicular to the radius vector). The flight path angle $\gamma$, namely the angle of the velocity vector with respect to the local horizon, given geometrically by $\tan \gamma=(d r / d \theta) / r$, is easily deduced from the shape equation (7.3):

$$
\tan \gamma=k_{1} k_{2} \cos \left(k_{2} \theta+\phi\right)
$$

Then, the shape equation (7.3), the equations of motion (7.6) and (7.7), and the tangential thrust assumption yield the following analytic expressions for the angular rate $\dot{\theta}$ and the thrust acceleration:

$$
\begin{aligned}
& \dot{\theta}^{2}=\left(\frac{\mu}{r^{3}}\right) \frac{1}{\tan ^{2} \gamma+k_{12 s}+1} \\
& a_{T}=\left(\frac{\mu}{r^{2}}\right) \frac{(-1)^{n} \tan \gamma}{2 \cos \gamma}\left[\frac{1}{\tan ^{2} \gamma+k_{12 s}+1}-\frac{k_{2}^{2}\left(1-2 k_{1} s\right)}{\left(\tan ^{2} \gamma+k_{12 s}+1\right)^{2}}\right]
\end{aligned}
$$

where $s \equiv \sin \left(k_{2} \theta+\phi\right)$ and $k_{12 s} \equiv k_{1} k_{2}^{2} s$. Last, the thrust angle is given by $\alpha=\gamma+n \pi$, where $n$ is an integer chosen so that the right-hand side of equation (7.10) is positive. When $n=0$, thrust is along the velocity vector; when $n=1$, thrust is in opposite direction. The derivations of the last two equations are quite laborious, and are therefore not shown here. On the other hand, since they are crucial equations in the computation of the trajectories, they are validated by the derivations included in appendix A. The circumferential $\left(v_{\theta}=r \dot{\theta}\right)$ and radial $\left(v_{r}=v_{\theta} \tan \gamma\right)$ speeds are provided by equations (7.8) and (7.9) as functions solely of position on the exponential sinusoid. Equation (7.10) similarly provides the thrust acceleration as a function of position.

A small remark can be made on the limitation of the exponential sinusoid when applied to an initially circular orbit. For a circular orbit it holds that $\dot{\theta}=\sqrt{\mu / r^{3}}$ and $\tan \gamma=0$. From equation (7.9) it follows that $k_{12 s}=0$, and together with equation (7.8) it results in $k_{1}=0$ or $k_{2}=0$. For these shape parameters it follows from the shape equation that the radius is constant with $\theta$ : a circular orbit. Therefore an exponential sinusoid is unable to simulate an orbit that gradually recedes from an initially circular orbit.

\subsubsection{Out-of-Plane Motion}

As explained before, the exponential sinusoids are pure planar shapes, and are assumed to lie in the orbital plane of the starting planet. The target planet does not lie exactly in this plane, but since the inclinations of the planets are relatively small $\left(3.39^{\circ}\right.$ for Venus is the largest of the relevant planets [Wakker, 1997a]), an approximate method can be devised to determine the out-of-plane thrust. The target's out-of-plane position at the time of the in-plane encounter is matched by using an additional thrust acceleration $a_{\text {out }}$ acting along or against the spacecraft's angular momentum vector. This out-of-plane acceleration is assumed to have the form

$$
a_{\text {out }}=c_{T} \frac{\mu}{r^{2}}
$$


with $c_{T}$ a constant. The positive $a_{\text {out }}$ is taken along the angular momentum vector. The form is chosen so that $a_{\text {out }}$ is inversely proportional to $r^{2}$, conforming to the decrease of received solar power. Because the out-of-plane acceleration will be much smaller than the acceleration due to the gravitational attraction of the Sun, the in-plane components of the specific angular momentum, $H_{x}$ and $H_{y}$, behave according to

$$
\begin{aligned}
\frac{\mathrm{d} H_{x}}{\mathrm{~d} \theta} & \approx \frac{r a_{\text {out }} \sin \theta}{\dot{\theta}} \\
\frac{\mathrm{d} H_{y}}{\mathrm{~d} \theta} & \approx-\frac{r a_{\text {out }} \cos \theta}{\dot{\theta}}
\end{aligned}
$$

where the $x$ direction is taken as lying along $\theta=0$. The chain rule for differentiation is used to obtain these equations. These in-plane components remain small in comparison to the out-of-plane component, since the required plane change is small. Therefore the total angular momentum remains approximately equal to just the out-of-plane component $H_{z}=r^{2} \dot{\theta}$, computed as if $a_{\text {out }}$ were zero. Using this approximation, expressions can be found for the out-of-plane angle $\psi$ and the out-of-plane velocity $V_{z}$ (see figure 7.3). These can be derived by using the fact that the dot-product of the position vector $\mathbf{r}$ and the angular momentum vector $\mathbf{H}$ should be zero, and likewise $\mathbf{V} \cdot \mathbf{H}=0$. This results in

$$
\begin{aligned}
\tan \psi & \approx r_{z} / r=-\frac{r_{x} H_{x}+r_{y} H_{y}}{r H_{z}}=-\frac{H_{x} \cos \theta+H_{y} \sin \theta}{r^{2} \dot{\theta}} \\
V_{z} & =-\frac{V_{x} H_{x}+V_{y} H_{y}}{H_{z}} \\
& \approx\left[H_{x}(\sin \theta-\tan \gamma \cos \theta)-H_{y}(\cos \theta+\tan \gamma \sin \theta)\right] / r
\end{aligned}
$$

in which the expressions for $V_{x}$ and $V_{y}$ follow directly from figure 7.3, while keeping in mind that the out-of-plane angle is small, so $r_{x y} \approx r$ and $V_{x y} \approx V$. Equations (7.12) and (7.13) can be substituted in equations (7.14) and (7.15) to result in:

$$
\begin{aligned}
\tan \psi_{f} & \approx c_{T} \frac{\mu}{r_{f}^{2} \dot{\theta}_{f}}\left[-I_{x} \cos \theta_{f}+I_{y} \sin \theta_{f}\right] \\
V_{z_{f}} & \approx c_{T} \frac{\mu}{r_{f}}\left[I_{x}\left(\sin \theta_{f}-\tan \gamma_{f} \cos \theta_{f}\right)+I_{y}\left(\cos \theta_{f}+\tan \gamma_{f} \sin \theta_{f}\right)\right]
\end{aligned}
$$

where subscript $f$ denotes quantities at the end of the thrust arc and

$$
\begin{aligned}
I_{x} & =\int_{\theta_{i}}^{\theta_{f}} \frac{\sin \theta}{r \dot{\theta}} \mathrm{d} \theta \\
I_{y} & =\int_{\theta_{i}}^{\theta_{f}} \frac{\cos \theta}{r \dot{\theta}} \mathrm{d} \theta
\end{aligned}
$$

These integrals are needed to compute the in-plane components of the angular momentum at the end conditions, as these are described by their derivatives with respect to $\theta$ (see equations (7.12) and (7.13)). The integrals can be computed numerically, after which equation (7.16) can be evaluated for the actually found out-of-plane angle, from which the constant $c_{T}$ can be determined. This is followed by the evaluation of equation (7.17), to quantify the out-of-plane velocity, 


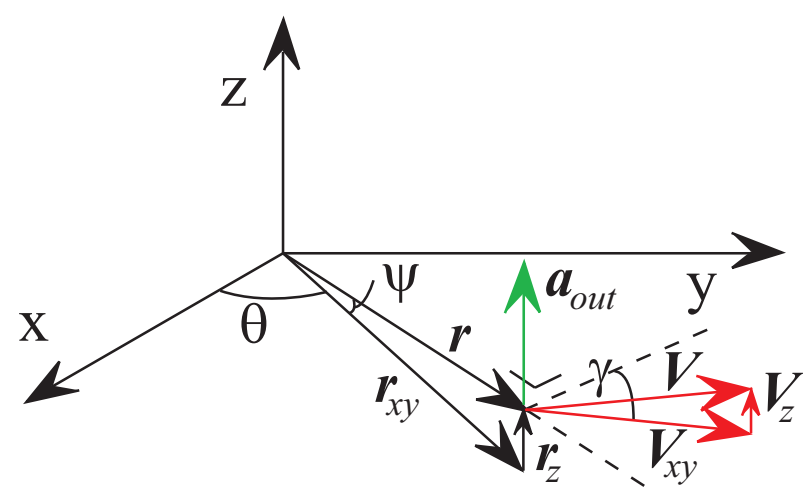

Figure 7.3 Visual representation of out-of-plane thrusting. $\psi$ is small, so $\left|\mathbf{r}_{x y}\right| \approx|\mathbf{r}|$ and $\left|\mathbf{V}_{x y}\right| \approx|\mathbf{V}|$.

which needs to be taken into account when computing the relative velocity of the spacecraft with respect to the target planet.

The effect of the out-of-plane motion on the TOF is ignored. This approximate method permits a rapid computation and is increasingly accurate for smaller outof-plane excursions. This method is developed with regard neither for attaining the most satisfactory thrust profile nor for the benefits to be had by using the gravity assists in a more efficient way. Therefore not too much consideration should be given to the out-of-plane thrust and the associated propellant. But to ensure the physical relevance of the computed trajectory, it can certainly not be ignored.

\subsection{Algorithm}

Now the shape and associated dynamics are given, it can be described how this should be incorporated in a computational program. As an input, the program needs a launch date and a value for the hyperbolic excess velocity $V_{\infty}$, both magnitude and direction (which is assumed to lie in the instantaneous orbital plane of the Earth). In the case of a flyby, it is assumed that it puts the spacecraft in the flyby body's orbit, while the turn angle can be varied by varying the altitude (see equation (3.25)). The instantaneous orbital plane of the starting planet for each leg defines the so-called low-thrust reference plane. For both cases (launch and flyby) a heliocentric velocity vector can now be found, which means that $\tan \gamma$ and $\dot{\theta}$ are known. Hence, the quantity

$$
k_{12 s} \equiv k_{1} k_{2}^{2} s
$$

can be determined from equation (7.9). Then, together with equation (7.8), this results into the constraint relationship

$$
k_{1}^{2} k_{2}^{4}-k_{2}^{2} \tan ^{2} \gamma-k_{12 s}^{2}=0
$$

between the as yet undetermined shape parameters $k_{1}$ and $k_{2}$. Thus, there remains one free-shape parameter, taken as $k_{2}$, which is used to target the next body.

The search over $k_{2}$ is narrowed in several ways. First of all, without loss of generality, only positive values are considered for $k_{1}$ and $k_{2}$. To avoid singularities around periapsis, it is required that

$$
1-k_{1} k_{2}^{2}>0
$$


which ensures that the denominator in equation (7.9) is always positive (when zero or negative, the exponential sinusoid cannot be followed using tangential thrust). For practical purposes, upper limits of one and two are imposed on $k_{2}$ and $k_{1}$, respectively, because the required thrust levels become infinite when $1-k_{1} k_{2}^{2}$ goes to zero (see equation (7.9) again). These are not absolutely fixed upper limits, but it turned out that they serve well for the problem at hand. The lower limit on $k_{2}$ is taken as 0.01, as this already permits 50 revolutions around the Sun between periapsis and apoapsis (see equation (7.4)), a number not likely to be exceeded in practice. Another lower limit on $k_{2}$ follows from the upper limit on $k_{1}$ and the constraint equation (7.21). Last, an upper limit on $k_{2}$ can be determined for which the resulting exponential sinusoid at least intersects the projection onto the low-thrust reference plane of the target body's orbit. For an outbound target, that is, those whose minimum projected radius $r_{t_{m i n}}$ is greater than the current radius $r_{B}=k_{0} e^{k_{1} s}$, this results in the following inequality

$$
r_{t_{\min }} \leq r_{\max }=k_{0} e^{k_{1}}
$$

Using again constraint relation (7.21) and applying some algebraic manipulation (limited to adding, dividing, squaring, and taking a natural logarithm), it can be rewritten to

$$
k_{2}^{2} \leq \frac{\tan ^{2} \gamma-2 k_{12 s} \ln \left(r_{t_{\min }} / r_{B}\right)}{\left[\ln \left(r_{t_{\text {min }}} / r_{B}\right)\right]^{2}}
$$

For inbound targets, that is, those whose maximum projected radius $r_{t_{\max }}$ is less than the current radius, it can be similarly obtained that

$$
k_{2}^{2} \leq \frac{\tan ^{2} \gamma+2 k_{12 s} \ln \left(r_{B} / r_{t_{\max }}\right)}{\left[\ln \left(r_{B} / r_{t_{\max }}\right)\right]^{2}}
$$

It might very well be the case that the right-hand side of equation (7.24) or (7.25) is negative. This simply indicates that the target planet cannot be reached from the current conditions via an exponential sinusoid. This is due to the fact that the initial velocity is too low (in the case of an outbound target) or too high (in case of an inbound target), which can be deduced from equation (7.9) and equation (7.24) or (7.25). This rounds up the discussion about the establishment of a range of $k_{2}$-values.

Thus, for each set of launch date and hyperbolic excess velocity (in the case of a launch) or for each turn angle (in the case of a flyby) a range of $k_{2}$-values is established that yield intersections of the exponential sinusoid and the orbit of the target. Then for each $k_{2}$-value the shape is uniquely determined. The shape parameter $k_{1}$ can be determined from the constraint equation (7.21), using the found value for $k_{2}$. Then the value for $s \equiv \sin \left(k_{2} \theta+\phi\right)$ can be found in the initial point (position of launch or flyby planet) using equation (7.20). $\theta$ is found from the geometry at the initial point (with $\theta=0$ at the ascending node of the low-thrust reference plane with respect to the ecliptic plane, and in the case that the starting body of a particular leg is the Earth, $\theta=0$ at the vernal equinox) and therefore the phase angle $\phi$ can be directly determined from $s$. From the shape equation at the initial point the value for $k_{0}$ can be determined. All that remains is to solve for the specific value of $k_{2}$, if any, which yields the correct Time Of Flight (TOF) to the intersection. This value is found by stepping through the range of $k_{2}$ 
values and at each step computing the location of the intersection point. Because no analytic solution is available, the intersection point is computed numerically. This is described in the first following subsection. The TOF is then computed by quadrature. It comprises of integrating the following equation numerically, which can be derived from equation (7.9)

$$
\mathrm{d} t=\frac{1}{\sqrt{\mu}} k_{0}^{3 / 2} e^{3 k_{1} s / 2} \sqrt{-k_{12 s}^{2} / k_{2}^{2}+k_{12 s}+k_{1}^{2} k_{2}^{2}+1} \mathrm{~d} \theta
$$

From the TOF a miss angle is computed, that is, the spacecraft-Sun-target angle when the spacecraft reaches the intersection point. Thus, a zero miss angle is searched for in the interval determined for $k_{2}$. For each initial condition one solution will be found. Now all the shape parameters are known, the delta-V can be determined by using quadrature on the thrust acceleration (equation (7.10)). Assuming a constant specific impulse and using Tsiolkowsky's equation (5.4), the propellant mass can be computed as a fraction of the initial mass.

\subsubsection{Finding Intersections}

In [Petropoulos and Longuski, 2004] a method to find intersections is explained which uses mean orbital elements to determine the position of the relevant planets. Since the more exact ephemerides of the JPL DE200 series have already been applied in the computation of the high-thrust trajectories (see section 6.1.1), this way of position and velocity determination of the planets will also be used in this case. First, a table is made, containing the projection of the target body's orbit on the low-thrust reference plane. This is done by stepping for a number of revolutions through the exact positions of the target body, and computing the projected radius $r_{t}$ and the associated polar angle $\theta_{t}$. The objective is obviously to find the polar angle for which the tabulated radius of the target body and the analytically solvable radius of the spacecraft $r_{s}$ on the exponential sinusoid or conic match. This is done by a step-controlled Newton-Raphson method (see equation (6.30)) to solve for the root of the difference $d_{i}$ in the inverses of the radii:

$$
d_{i}(\theta)=1 / r_{t}(\theta)-1 / r_{s}(\theta)=0
$$

The derivative needed for the Newton-Raphson methods is simply

$$
\frac{\mathrm{d} d_{i}}{\mathrm{~d} \theta}=-\frac{1}{r_{t}^{2}} \frac{\mathrm{d} r_{t}}{\mathrm{~d} \theta}+\frac{1}{r_{s}^{2}} \frac{\mathrm{d} r_{s}}{\mathrm{~d} \theta}
$$

The inverse radius is used because it renders faster computations [Petropoulos and Longuski, 2004]. As an initial guess for $\theta$, the easily determined value where the analytical shape radius is equal to the minimum projected radius of the target orbit (for an outbound intersection) is taken. In figure 7.4 this minimum projected radius is displayed by the straight black line. As well in this figure are multiple exponential sinusoids with equal starting conditions, but varying values for $k_{2}$. In section 7.4 this figure will be elaborated. It might be that in the first iteration step $\theta$ becomes smaller. This should not be the case since from the geometry it is clear that $\theta$ should increase to find an intersection. In that case the mentioned step-control needs to be invoked. It turned out that setting the next initial guess $\pi / 2$ beyond the first one, ensures that the intersection can be found. 


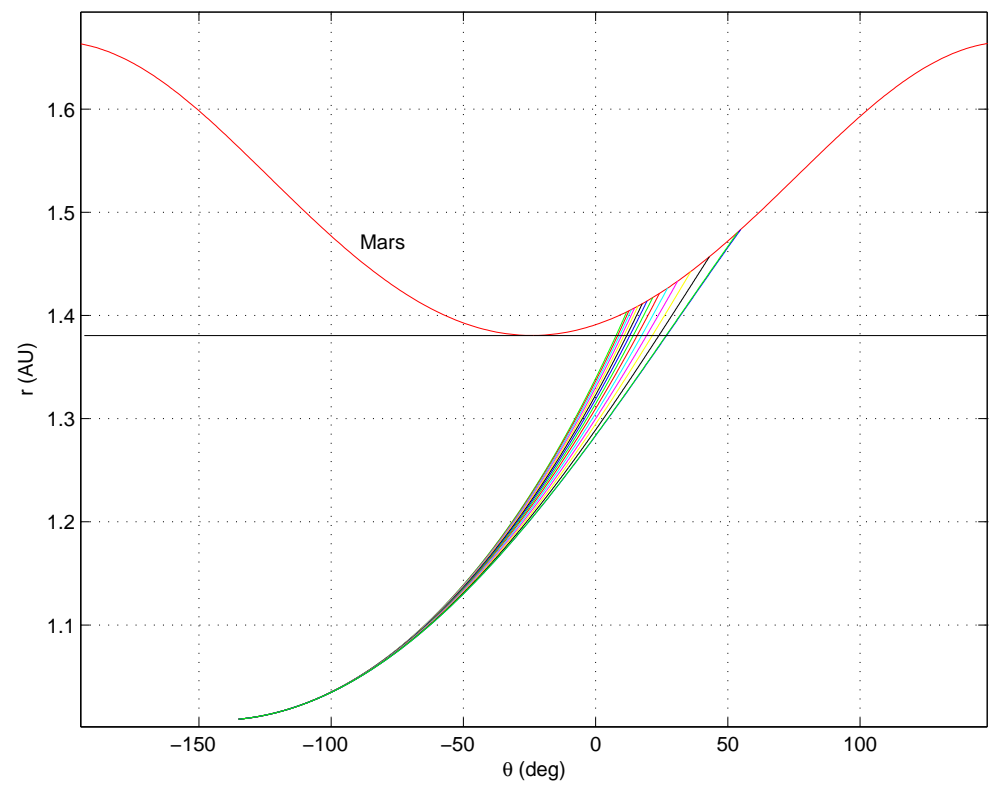

Figure 7.4 Intersections of multiple exponential sinusoids with the orbit of Mars. The starting planet is the Earth on the $6^{\text {th }}$ of May, 2003, with a launch $V_{\infty}$ of $1.6 \mathrm{~km} / \mathrm{s}$.

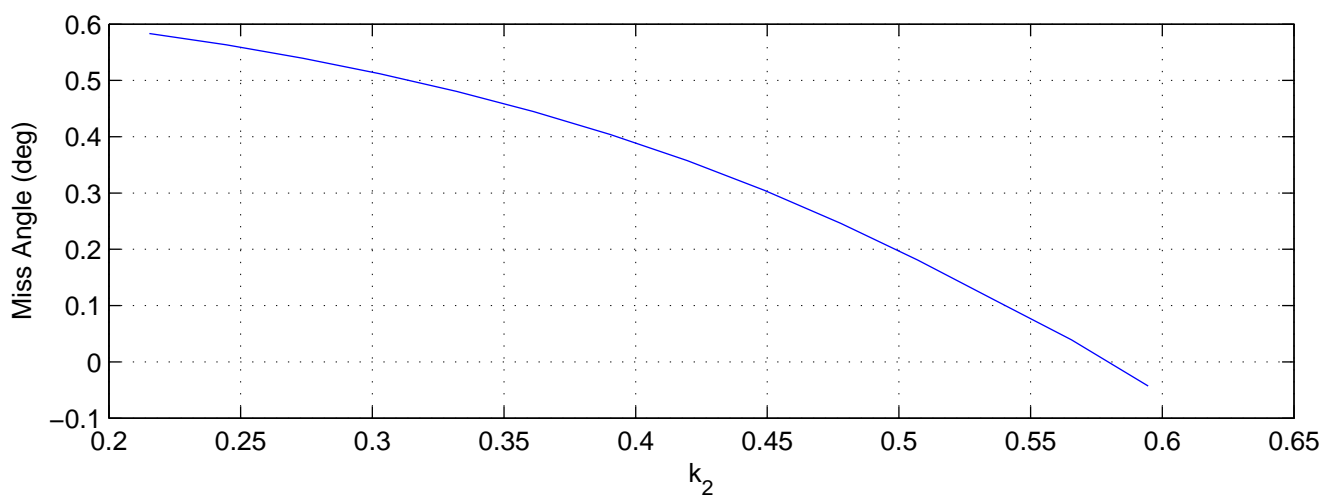

Figure 7.5 Miss angle as a function of $k_{2}$ for the Earth-Mars leg from figure 7.4.

\subsection{Verification}

In the paper by Petropoulos and Longuski [2004], some results have been indicated for a trajectory via Mars to the asteroid Ceres. The ephemerides of Ceres are not included in the JPL DE200 series, so the Mars-Ceres leg cannot be verified, but the Earth-Mars leg can provide a verification of the implementation of the exponential sinusoid. When stepping through the range of $k_{2}$-values, the resulting exponential sinusoids are shown in figure 7.4 for the initial conditions indicated by Petropoulos and Longuski [2004], which are restated in the caption of the figure. Computing the miss angles as described in section 7.3 results in figure 7.5. Noticeable is the quite limited range in the miss angle. This is due to the fact that the last part of the exponential sinusoid more or less resembles the orbit of Mars. Thus, the spacecraft approximately follows Mars, resulting in the small sensitivity of the miss angle. Fortunately, this is not the case for each set of initial conditions and geometry. In the case the exponential sinusoid roughly intersects the target body's orbit perpendicularly, the range of miss angle can be tens of degrees. From figure 7.5 
it can be seen that an actual Mars encounter (miss angle is equal to zero) occurs for the almost highest permissable $k_{2}$-value. This corresponds approximately to the right most exponential sinusoid in figure 7.4. For this trajectory a travel time is found by own calculations of 243 days. In the paper a travel time of 271 days is stated. This difference can be explained by the resembling orbits as well. A small deviation in the actual position of the target body, in the order of tenths of a degree, can already cause the travel time to deviate by the mentioned amount, as was found by a small sensitivity analysis. Since the positions are calculated differently (see section 7.3.1) this position difference could occur, especially when the given orbital elements given and used by Petropoulos and Longuski [2004] are only accurate to at the most 3 significant numbers. Even if their formulas to determine the position of the target body were implemented, this could not be further verified, since only 3 out of the 6 orbital elements are given by them.

Using equation (7.10) the thrust acceleration profile can be computed, and again compared to the one found by Petropoulos and Longuski [2004] (left-hand side of figure 7.6). The thrust profile found by own calculations can be seen on the right-hand side of figure 7.6. Just after the launch and just before the Mars encounter a part of the thrust profile is 'missing', due to the shorter time of flight, as explained earlier. But the general characteristics are definitely similar. Concluding, the found approximate correspondence between the travel times and the acceleration profiles gives a rather good insurance that the exponential sinusoid is correctly implemented.
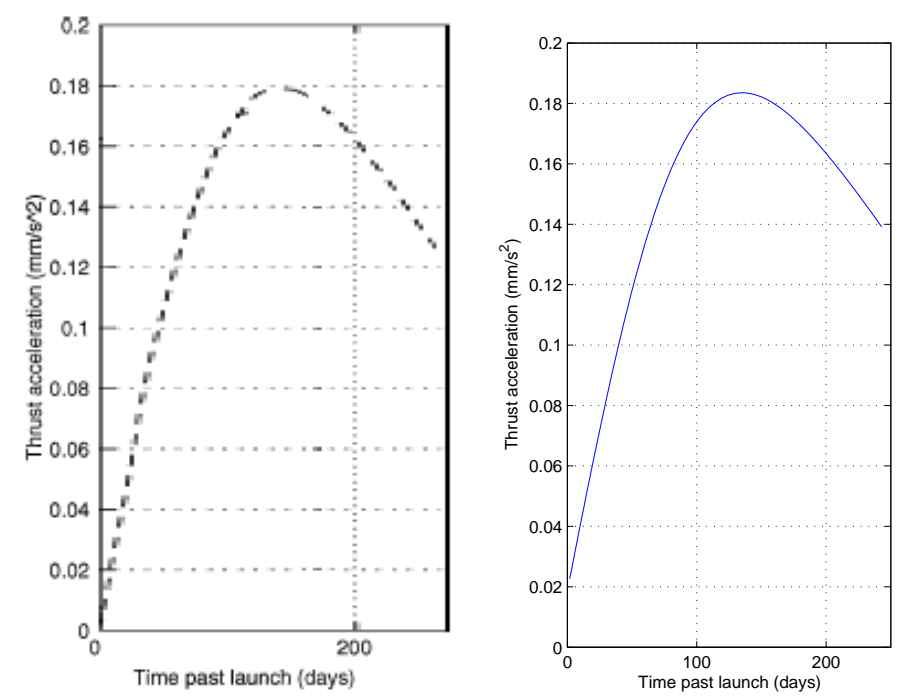

Figure 7.6 Thrust acceleration profile of an Earth-Mars leg according to Petropoulos and Longuski [2004] (left-hand side) and according to own calculations (right-hand side). 
Chapter 8

\section{Optimization}

Optimization is used throughout many design and logistics problems. One application is to design a spacecraft trajectory which performs better than all other possible trajectories: it needs to be optimized. Some parts of the last sentence can be further clarified. First of all, better means that the trajectory minimizes or maximizes an objective function, like a cost or energy function. Secondly, possible implies that there are some constraints that limit the search space, like the inability to fly directly through a planet. The last word optimized brings us to optimization: find the appropriate values for variables that minimize/maximize the objective function while satisfying the constraints. These variables can represent basically anything, from height to mass, and from launch date to steering angle. Optimization can be performed in several ways, which are explained in section 8.1. Then a selection will be made for the current problem, for which a more in-depth discussion is given in sections 8.2. How to treat the optimization of multiple objectives is point of discussion in section 8.3.

\subsection{Methods Overview}

Many different optimization methods exist, but not all of them are suitable for every type of problem. Their differences in finding the optimum has led to the following three main types of optimization methods [Goldberg, 1989]:

- Calculus-based

- Enumerative

- Random Search

These different methods are now discussed in more detail.

\subsubsection{Calculus-based Methods}

When an optimization method uses the derivatives of the objective function it is said to be calculus or gradient based. They subdivide into two main classes: indirect and direct. Indirect methods seek extrema by solving the equations resulting from setting the gradient of the objective function equal to zero. On the other hand, direct methods seek optima by moving in the direction of the largest 


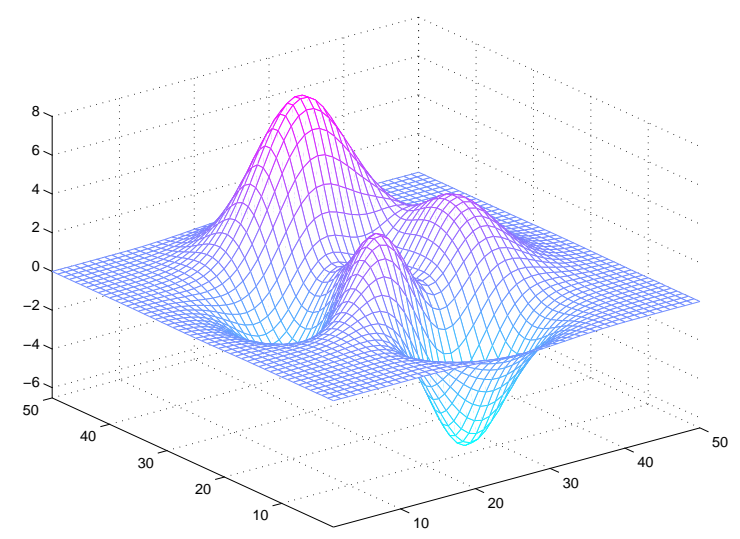

Figure 8.1 A multiple peak function:

$z=3 \cdot(1-x)^{2} \cdot e^{-x^{2}-(y+1)^{2}}-10 \cdot\left(x / 5-x^{3}-y^{5}\right) \cdot e^{-x^{2}-y^{2}}-1 / 3 \cdot e^{-(x+1)^{2}-y^{2}}$

gradient. These approaches only work well in the case of a smooth, differentiable objective function, which has only one local optimum (unimodal). The last part can be explained by using figure 8.1. In this figure there are multiple local maxima and minima. When searching for the global maximum, starting the search of zero-finding procedures in the neighborhood of one of the lower peaks will result in missing the highest peak. The same holds when one wants to minimize the objective function. In other words, for multimodal objective functions, the use of calculus-based methods will very likely result in the determination of a local optimum.

The required existence of derivatives also poses a serious problem. Many practical parameter spaces have little respect for the notion of a derivative and the smoothness this implies. Since the problem at hand is certainly not unimodal and has at the best a complicated set of derivatives and at the worst several undefined derivatives, calculus-based methods can be ruled out for finding a global optimum. They could be useful for further enhancing a found global optimum, but due to practical time constraints this will not be done in this thesis work and will form part of the recommendations.

\subsubsection{Enumerative Methods}

The idea of enumerative schemes is quite straightforward; within a search space the algorithm starts looking at objective function values at every point in the space, one at a time. Although the simplicity of this type of algorithm is attractive, such schemes are mostly rejected for one simple reason: lack of efficiency. The search space for the current and many other practical problems is simply too large to search one at a time. A more advanced form of enumerative techniques is Dynamic Programming (DP). This technique decomposes a problem that is hard to solve into a number of subproblems that are more likely to be solved. After the subsolutions have been found, they are combined to form the solution of the original problem. Although it can work quite well on relatively small problems, as soon as the size increases DP becomes very slow. Therefore enumerative techniques can be discarded as a whole on first sight. 


\subsubsection{Random Methods}

Now the previous two methods have been rejected, the only left possibility is a random method. Strictly random walks and schemes search the search space totally at random and save the found best. From this follows that they can not be expected to perform any better than enumerative methods. Fortunately other random methods exist that use random number generation in a less strict way. These search procedures use random choice as a tool to guide a highly exploitative search through a coding of a parameter space. This randomized search does not necessarily imply directionless search as is the case for a strictly random scheme. A search algorithm that makes use of this random choice is Simulated Annealing (SA). It mimics the annealing of metal, with the objective function viewed as the energy state of the metal, which has to be minimized. SA is much more effective than simple random search, but for large problems with many involved variables it still is quite time-consuming.

More time-efficient are algorithms based on evolution. They exploit the 'survival-of-the-fittest' principle, where a whole population of solutions gets the opportunity to evolve. This is a relatively young area of research, and therefore no generic name has been defined yet. Denominations like Genetic Algorithms, Evolution Programming and variations on the two are in use. In this thesis the term Evolution Programming (EvP) will be used primarily, although the term Genetic Algorithm (GA) will incidentally show up since it is part of the name of the used program (see chapter 9). Although EvP is relatively new, it has already proven to be very useful for optimizing complex, large problems. A testimony for this is the successful use of EvP for a significant amount of theses performed at the Faculty of Aerospace Engineering, Delft University of Technology [e.g., Schlijper, 2003; Garot, 2005]. Due to its capability to tackle large and complex problems, EvP will be the tool to optimize a trajectory to Neptune. This choice for EvP does not imply that the other techniques are not useful. The schemes mentioned and countless hybrid combinations and permutations have been used successfully in many applications; however, for the current problem EvP is considered the most useful. It will be discussed in the next two sections.

\subsection{Evolution Programs}

As mentioned in the previous section, EvP tries to find the optimal solution by means of evolution and survival of the fittest. In the classical format, the fittest individuals of the population have a higher probability to be chosen to produce offspring. After several generations the fitter individuals are most likely to prevail, and one comes closer to the optimum. In order for EvP to justify the earlier statement that it is superior to other search techniques for large and complex problems, it must differ on some very fundamental points. EvP is different from other optimization and search procedures in three ways:

1. Search from a population of points, not a single one.

2. Use payoff (objective function) information, not derivatives or other auxiliary knowledge.

3. Use probabilistic transition rules, not deterministic ones. 


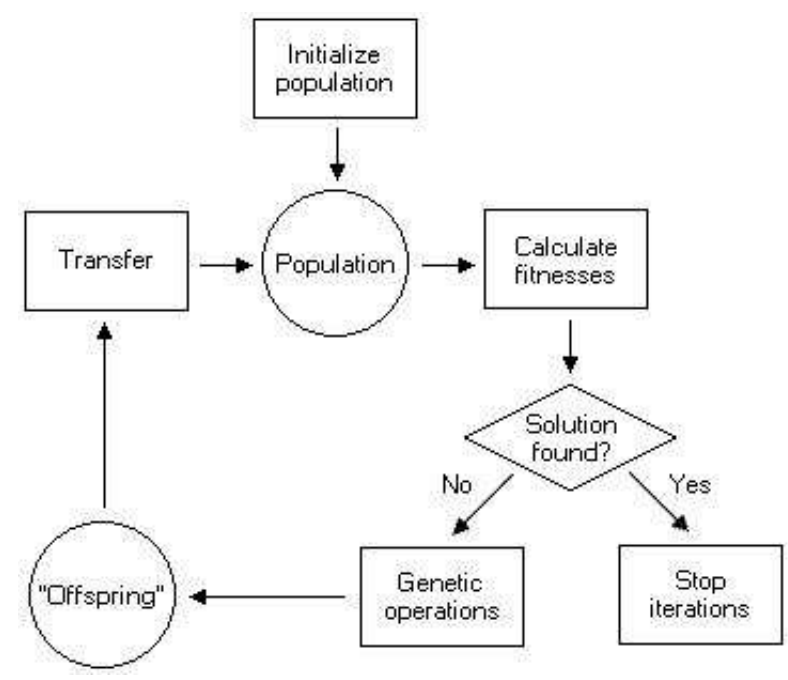

Figure 8.2 Flow chart illustrating the basic steps in Evolution Programs [Jakobsen, 2004]

An overview of the basic steps taken in an $\mathrm{EvP}$ optimization problem is given in figure 8.2. First an initial population has to be created. This is usually created at random, which is one of the strong points of EvP: a good starting point is not necessary. It might be time-efficient to start in the neighborhood of the optimum, but this could lead to premature convergence: early convergent behavior, without guarantee of optimality. Then the objective function is evaluated for each individual in the population, which renders the fitness. This is followed by checking objectively whether the global solution is found or not. If not, the iteration continues by applying the genetic operators, resulting in offspring. This offspring can now take the place of their parents and form the new population, which closes the loop.

The power of EvP resides in the aforementioned genetic operators. Therefore they deserve a further discussion. A classical approach consists of three genetic operators: selection, crossover and mutation. In the following it is explained what they comprise of in the context of this thesis work. Many variations on these operators exist, but were not applied in this work. For a discussion of these operators one is referred to my own literature report [Melman, 2006]. To enable a good understanding of the operators, the representation of the individuals is discussed first.

Representation As mentioned and seen before, EvP uses a coded representation of the solutions instead of the solutions themselves. Traditionally a binary representation is used, since it facilitates the theoretical analysis and allows relatively simple genetic operators. It works as follows: for simple integers the application is quite straightforward. For example, 21 would be coded as the string 10101 $\left(1 \cdot 2^{4}+0 \cdot 2^{3}+1 \cdot 2^{2}+0 \cdot 2^{1}+1 \cdot 2^{0}=21\right)$. Rational numbers can also be constructed by assigning a lower bound $x_{l}$ and an upper bound $x_{u}$ between which the variable $x$ can vary. From the string value $s$ and the number of binary digits per string $m$ the value for $x$ can be obtained:

$$
x=x_{l}+s \cdot \frac{x_{u}-x_{l}}{2^{m}-1}
$$


From formula 8.1 it can be seen that when one wants to increase the resolution, the number of bits per string $m$ has to be increased. For high-precision, multidimensional problems the required length of the binary solution vector will then go out of bound, rendering an enormous search space. For such problems the classical EvP will perform poorly, due to the bit representation. Therefore, it can be worthwhile to look at so-called floating point genes. The solution is then coded in an array of floating-point numbers and the resolution is not fixed. It moves the EvP closer to the problem space, which forces, but also allows, the operators to be more problem specific. For example, this representation has the property that two points close to each other in the representation space must also be close in the problem space, and vice versa, which is not necessarily true in the binary approach. Michalewicz [1996] has shown that for problems with large search domains, the floating-point representation is faster and can at the same time provide a higher precision. Therefore the floating-point representation is chosen in this thesis work.

Selection Every iteration step the values of the objective function(s) for the solutions have to be evaluated. Or, in natural terminology, each generation all individuals in the population are measured by their fitness. Then individuals, that may produce offspring, can be selected on the basis of this fitness in several ways. The most basic selection method is using a weighted roulette wheel. Each individual is assigned a wheel slot size proportional to its fitness (see figure 8.3). This roulette wheel can be turned a predefined number of times (equal to the desired population size) to sample a full population. Using this approach highly fit individuals have a higher chance of producing offspring. A very direct way of favoring the best is elitism, which makes sure the best individual(s) is/are preserved. It improves local search at the expense of global search, but when used modestly, this will give an overall better performance [Goldberg, 1989]. This selection method is implemented during this thesis work.

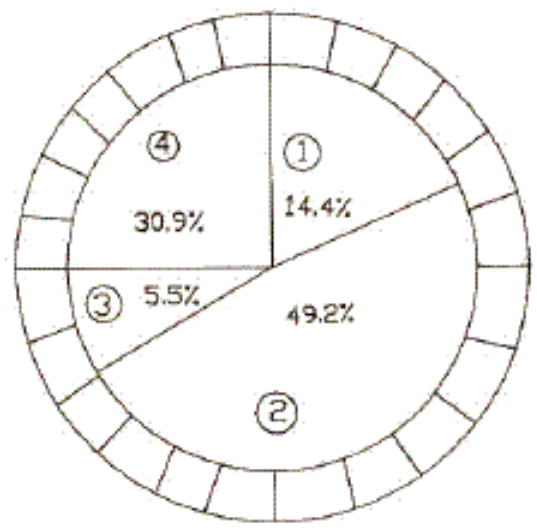

Figure 8.3 Weighted roulette wheel used for simple selection of offspring producing individuals [Goldberg, 1989] 
Crossover This is the main operator that creates the next generation from the selected individuals. As the individuals are represented by coded strings, the crossover operator can split up the 'parents' at some randomly defined location and recombine to form 'children'. In this way the genetic material of the mating individuals is mixed. For example, consider the crossover of strings $A$ and $B$ after the 3rd position:

$$
\begin{aligned}
& A=352 \mid 63 \\
& B=432 \mid 42
\end{aligned} \Rightarrow \begin{aligned}
& A^{\prime}=35242 \\
& B^{\prime}=43263
\end{aligned}
$$

In the case of a floating-point representation, a new individual $\mathbf{c}$ can be created from two parents $\mathbf{a}$ and $\mathbf{b}$ by using arithmetic crossover [Michalewicz, 1996]:

$$
\mathbf{c}=q \cdot \mathbf{a}+(1-q) \cdot \mathbf{b}
$$

with a random value $q \in[0,1]$. For this thesis a hybrid version is taken: take the first $r-1$ parameters of the first randomly chosen individual and the parameters on positions $r+1$ up to and including the last parameter of the second randomly chosen individual. The parameter on position $r$ of the offspring is created by arithmetic crossover between the two parents. For example, with $r=3$ and $q=0.7$ and the parents being

$$
\begin{aligned}
\mathbf{a} & =\left[\begin{array}{llll}
1.6 & 3.4 & 5.6 & 2.3
\end{array}\right] \\
\mathbf{b} & =\left[\begin{array}{llll}
3.8 & 2.6 & 6.6 & 1.2
\end{array}\right]
\end{aligned}
$$

the resulting offspring is

$$
\mathbf{c}=\left[\begin{array}{llll}
1.6 & 3.4 & 5.9 & 1.2
\end{array}\right]
$$

Mutation Even though selection and crossover effectively search and recombine solutions, mutation is helpful. Although it does play a secondary role, it keeps a population diverse. Mutation can thus be seen as an insurance policy against premature loss of important notions. It is executed by sometimes altering a small part of a solution. In the simple case this occurs randomly with a constant probability $p_{m}$. Its value is usually very small, less than one percent. In this thesis work, so-called immigration is implemented, an operator similar to mutation. Just like mutation, it ensures population diversity and prevents premature convergence. Immigration introduces entirely new individuals each generation. The number of immigrants can be a constant or varying with population size and/or generation number. In this thesis a constant fraction of a new population consists of immigrants.

\subsection{Multi-Objective Optimization}

Often when designing interplanetary missions, several criteria need to be considered. While final spacecraft mass is the typical measure of performance, it is not the only desirable trait. Minimizing time of flight is quite often an equally attractive mission objective, and one that is inversely related to maximizing payload mass, which is equivalent to minimizing the propellant used. Other objectives may also arise in the mission planning process that are deemed equally advantageous. Combining these objectives forms a multi-objective optimization problem. 
A common method for handling multiple objectives is to combine them into a single scalar objective $F$ by evaluating each sub-objective $f_{j}$ and then weighing its influence to the overall objective through a weighting factor $w_{j}$

$$
F(\mathbf{a})=\sum_{j=1}^{k} w_{j} f_{j}(\mathbf{a})
$$

Such reduction techniques eliminate the need for a more complex multi-objective algorithm, but introduce new parameters in the form of weighting factors. Individual solutions are highly sensitive to the magnitudes of these weighting factors. The user must become familiar with the exact relationship between objectives in order to determine the proper weighting values that will yield the really desired result. Improper assignment of weighting factors can result in a bias towards certain objectives. Determination of the correct weighting factors can, in fact, become an optimization process of its own.

In addition, the result of the single-objective optimization will be a single optimal solution rather than a set of optimal solutions that demonstrate the trades between different objectives. This is acceptable for cases in which optimality of all objectives coincide in the same solution, but in most cases where adjustments beneficial to one objective are detrimental to others, such a technique provides only a single point on what may be a large set of possible solutions. In the case of EvP, such a formulation fails to take advantage of the population-based nature of the technique. It therefore becomes desirable to develop a more robust, multiobjective algorithm, capable of identifying the relationships between objectives and able to make better use of the population-based EvP to produce complete sets of optimal solutions. The criteria for having an optimal solution or not will be discussed in the following.

Pareto optimization, named after Italian sociologist and economist Vilfredo Pareto (1848-1923), is the principle of optimizing multiple competing objectives. The Pareto-optimal set is a family of points which is optimal in the sense that no improvement can be achieved in any objective without degradation in others. The following formulas can be used to explain this further

$$
f_{i}(\mathbf{a}) \leq f_{i}(\mathbf{b}) \forall 1 \leq i \leq k \quad ; \quad f_{i}(\mathbf{a})<f_{i}(\mathbf{b}) \exists 1 \leq i \leq k
$$

In the case of minimization, individual $\mathbf{b}$ is said to be dominated by $\mathbf{a}$ if the inequalities of 8.4 hold. Individual $\mathbf{b}$ performs worse than or equal to individual a on all objective functions. If an individual is not dominated by any of the individuals in the population it is called Pareto-optimal or non-dominated. All these non-dominated individuals together form the optimal Pareto front. It is the intention of multi-objective optimization to find this Pareto front. How to find this Pareto front using EvP is discussed in [Melman, 2006]. However, in this thesis work multi-objective optimization is not implemented, but instead the time aspect was introduced by constraining the solutions to be within a certain time interval (see section 10.1). It would certainly be beneficial to introduce the multi-objective optimization, and therefore it will form part of the recommendations. 

Chapter 9

\section{Galomusit}

To design an interplanetary spacecraft trajectory takes many calculations; to do this by hand would be very cumbersome. Therefore a software tool is needed. To use off-the-shelf software, where one only has to push some buttons to come to an answer, does not give the intellectual challenge that should be part of a graduation thesis. On the other hand, to completely develop a new program by yourself can take a considerable amount of time, which could negatively affect the amount of time available for generating and discussing useful results. Fortunately, within the Faculty of Aerospace Engineering, Delft, already a software tool exists that can handle multiple-swing-by interplanetary trajectories, named Galomusit. But luckily still many adaptations remained to be done to the original program, which guarantees that the intellectual challenge is still present. In the course of this chapter the history of Galomusit (section 9.1), the general structure of the program (section 9.2) and the numerous adaptations (section 9.3) will be made clear. To what values the optimization procedure is set is discussed in section 9.4. The chapter concludes with a verification of Galomusit in section 9.5.

\subsection{History}

It all started in 2002/2003 when R.M.H. Schlijper wrote Galomusit for his own graduation project. The goal was to optimize a flight to Pluto within the 20052020 time frame. For that matter a genetic algorithm (see chapter 8) and Lambert targeting (see section 6.2) were put into the program. But it was a very specific application, since it could only deal with some predefined paths to Pluto. Schlijper also conceived the program's name Galomusit: Genetic ALgorithm Optimization of a MUltiple Swing-by Interplanetary Trajectory. After Schlijper it was used and adapted by two other students, which were supervised by ir. R. Noomen, just like Schlijper and myself. First it was adapted by L.J. de Jong in 2003/2004 to deal with aerogravity assists. Also his assignment was to fly to Pluto. As the last of my predecessors, Namik Erdogan changed it, so it could be applied for a manned Mars mission. The genetic algorithm was not used, instead an enumerative search was executed. The contribution of these three persons resulted in a program with many different 'flavors', giving me the challenge to make the program more consistent and universal. 


\subsection{General Structure}

The structure of Galomusit can best be explained by means of a flow diagram, see figure 9.1. Logically the program requires input, which comprises both the mission layout and the genetic optimization parameters (see section 9.4). Then a first generation can be made with the individuals in fact being a string of dates: the first represents the date of launch, the last the date of arrival, and the intermediate ones the dates for the swing-bys. How these were (in the old version) and are (in the new version) generated is treated in section 9.3.1. This forms the first initiation step. Then the individuals go one by one through the evaluation step. Here the problem model comes into play. First the positions of the planets at the particular dates are determined using the JPL DE200 ephemerides (see section 6.1.1). For each leg, with the end positions and time of flight defined, the associated shape can be determined using the Lambert targeting (see section 6.2). The delta-V's associated with the launch and capture can be determined using equations (6.2) and (6.3), respectively. For which parking orbits these delta-V's need to be computed can be defined directly in the input subroutine. The orbits used in this thesis work will be stated in section 10.1. The delta-V('s) associated with the swing-by(s) are found using the information from section 6.3. The total delta- $\mathrm{V}$ is then directed to the main program again. Namely, this delta- $\mathrm{V}$ value is what is actually optimized for. It represents the energy requirements for a particular trajectory. Hereby it also reflects the final mass of the spacecraft: from equation (5.5) it follows that

$$
m_{f}=m_{i} e^{-\Delta V / V_{e}}
$$

Thus, the lower this delta-V, the higher the final mass. Coming back to the program, it is checked whether the found delta- $\mathrm{V}$ can be allowed. Excessively high delta-V's should be prevented from entering the population, since they cannot form the basis of a good solution. On the other hand, also moderately bad individuals should be tolerated, since they could evolve into better ones. In this thesis work it is chosen to set an upper bound of $100 \mathrm{~km} / \mathrm{s}$. If this condition is met, the next individual can be evaluated. If not, a new individual should be generated in the initiation procedure. From this follows that the initiation part in the flow diagram can represent both the generation of a whole new population and the generation of a single individual. After a full population has been evaluated, the convergence of the population's fitness is checked (see section 9.4). If there is no convergence yet, a new generation will be generated and the computations are done again as mentioned earlier. If there is convergence, the optimum is found and the program stops, but not after writing some useful properties of the best individual(s) to an output file.

\subsection{Adaptations to Galomusit}

Generally speaking, the old version of the program has been restyled to be more user-friendly. This has been attained partly by including many more comments to explain to the user why these particular computations/actions are actually taken/done. But more important is the clustering of all the input in one subroutine, instead of being spread over all subroutines. This enables a new user to be up and running much faster than before. For example, one can specify in this subroutine 


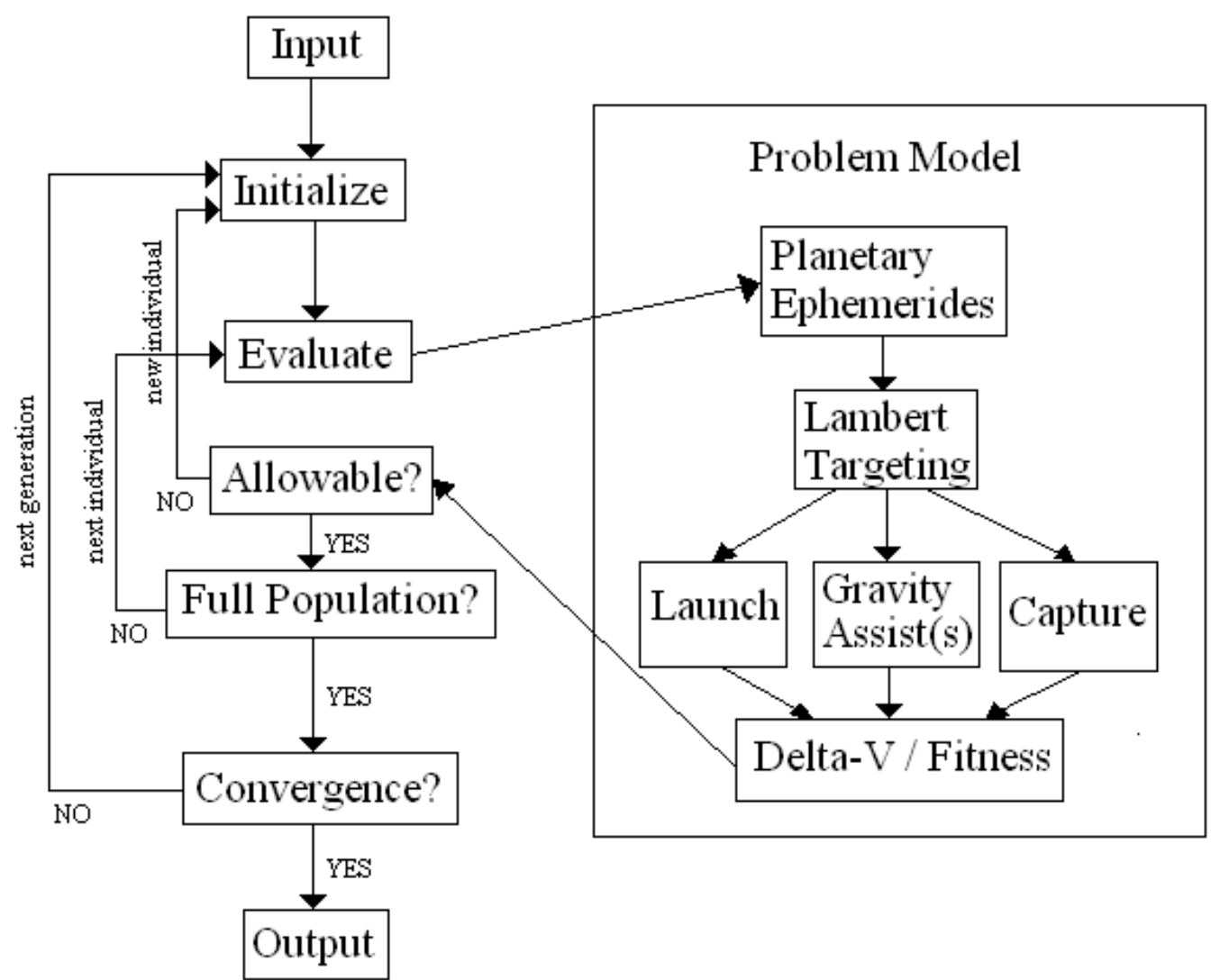

Figure 9.1 Flow diagram of Galomusit.

whether one wants to perform a flyby mission (without capture) or an orbiter mission (with capture) by changing one single identifier, instead of going to the capture subroutine and setting the resulting delta- $\mathrm{V}$ equal to zero (in case of a flyby mission). Also, the old version was specifically made to optimize a flight from Earth to Pluto. In its present state it can deal with a flight from any planet in the Solar system to any other one, by just changing two input parameters.

Furthermore, more arraying has been implemented, which changed for example the length of a part of a particular subroutine (while still keeping the same functionality) from 75 to 12 lines. Another improvement has been the automation of the program. By once running the program it can optimize multiple sequences instead of just one, while writing the output of different sequences to different files. Since there were many different possible sequences, this proved to be very useful. Additionally, the computation of a powered swing-by has been changed. In [Schlijper, 2003] three different components of the delta-V were discussed in relation to a swing-by, while in this thesis it has been argued that at the most two distinct components are needed (see section 6.3). Also, it can now be specified whether an aerogravity assist is allowed to take place at a certain planet. But if the patching of the heliocentric legs can be done without an AGA, this option will be taken, resulting in a pure gravity assist.

The most important improvement, which significantly reduced the computation time, will be discussed in the remainder of this section. 


\subsubsection{Generation of Individuals}

In the old version of Galomusit the method of generating individuals was to define a date for each planet encounter within the full range between minimum launch date and maximum arrival date. This was followed by a check whether these dates were consecutive. In the case of multiple swing-bys the chance of actually finding all encounter dates to be consecutive in the right order decreases exponentially. Although generating these dates goes very fast, and checking the chronology as well, it has to be done many times, and it severely slows down the computation. Therefore a more sensible approach is taken to generate individuals, implementing problem-specific knowledge. First of all, the launch date should not lie too close to the maximum date of arrival, since no sufficient time would be left to make the transfer. Therefore also an upper limit on the launch date has to be defined. The date for the next encounter now has as a lower limit the first found date plus a lower limit on the expected time of flight. Also an upper limit is defined to prevent for example an excessive transfer time of 15 years between Earth and Venus. This upper limit is taken to be directly proportional to the Hohmann transfer time between the relevant planets. A factor of 4 is used, to enable also the finding of heliocentric legs with a semi-major axis considerably larger than the semi-major axes of the involved planets. If this upper limit exceeds the maximum date of arrival, obviously this last date is taken as the upper limit.

In the case of a consecutive encounter with the same planet, this changes a little bit. The time the satellite flies between the first and the second encounter of the same planet should be at least more than one revolution of the involved planet, otherwise the orbit of the satellite would match the planet's orbit. The idea is to make an intermediate transfer with travel time approximately two times higher, so that the satellite has approximately made one revolution about the Sun, while the planet made two. Therefore the date is forced to be between 1.5 and 2.5 times the planet's orbital period after the first date of encounter. In this way a particular planet can be used more than once.

By using this new approach of generating individuals the computation time was drastically reduced, especially for multiple-swing-by trajectories. For example, both programs were run for a trajectory making 4 swing-bys, so a total of 6 dates (launch and arrival date included). The old version took approximately 12 hours, while the new version was finished within 10 minutes. Trying to find a solution with 5 swing-bys with the old version did not give any results within a day, while the new version takes only about 20 minutes. This is obviously a significant reduction in computation time, which proved to be very useful, since many different mission sequences had to be optimized.

\subsection{Setting the Evolution Program}

As is obvious from chapter 8, optimization using EvP is not so straightforward as applying an enumerative technique. Multiple parameters need to be set before the optimization can start. The value of a certain parameter is strongly problemdependent. Therefore a small study has to be performed to determine the values to use. 
First of all, elitism is applied, which makes sure the best individual(s) is/are preserved. A small survey indicated that percentages above about $20 \%$ resulted in a rapid convergence, but generally not towards the global optimum. For percentages of $0-20 \%$ the results did not deviate substantially from each other. The choice has been made for this thesis to preserve the best $10 \%$ of the individuals. Immigration also plays a role in Galomusit. It introduces entirely new species in the population, thereby ensuring population diversity and preventing premature convergence. Logically the number of immigrants should not become too large or the power of evolution programs would be lost and an enumerative optimization scheme results. Again the choice has been made to use a percentage of $10 \%$. This leaves a fraction of 0.8 to be formed by recombination of individuals of the previous population.

Another parameter is the number of generations. It follows in fact from the degree of convergence. When it was found that the population's fitness did not change significantly over a particular number of generations, the evolution program stopped, since apparently it could not find a better individual. To keep computing would then only degrade the effectiveness of the program. Using a trial-and-error approach the following parameters were found: convergence check over 10 generations, maximum fitness value not changed by more than $0.1 \%$ and the average fitness value not by more than $0.5 \%$. In about $90 \%$ of the cases this resulted in a total number of generations of less than 100. Complementary to this discussion is figure 9.2. Its left-hand side displays the delta- $\mathrm{V}$ as a function of the generation number for a specific mission to Neptune. From the first generation to the last one, the delta- $\mathrm{V}$ decreases by approximately a factor two. At first the delta- $\mathrm{V}$ decreases quite rapidly, after which it more or less converges. But still a steady improvement is obtained, as displayed by the detail on the right-hand side. If less stringent parameters would have been taken, a final delta- $\mathrm{V}$ of $6.72 \mathrm{~m} / \mathrm{s}$, or even $7.04 \mathrm{~m} / \mathrm{s}$, would have been found, instead of $6.67 \mathrm{~m} / \mathrm{s}$. This rationalizes the choice for the mentioned parameters, although the exact values remain somewhat arbitrary.
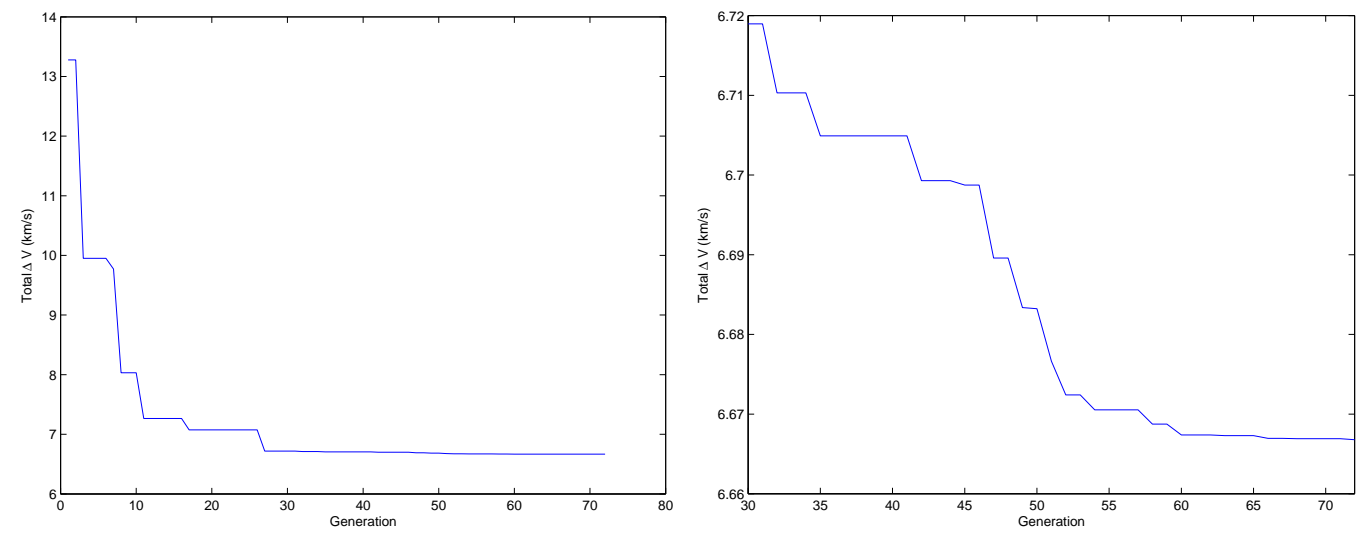

Figure 9.2 Fitness (portrayed by $\Delta V$ ) as a function of generation for a mission to Neptune via Venus, Earth, and Jupiter. The figure at the right-hand side is a detail of the one at the left-hand side.

The final parameter which needs to be set is the population size. According to [Biesbroek and Ancarola, 2003] this is the most influential parameter. This is also supported by own findings, which can be seen in table 9.1. For 5 different sequences (each with a different number of flybys) three runs are executed with 


\begin{tabular}{|r|c|ccc|c|c|}
\hline Swingby & Population & \multicolumn{3}{|c|}{$\Delta V[\mathrm{~km} / \mathrm{s}]$} & & \\
Sequence & Size & Run 1 & Run 2 & Run 3 & Margin & Used \\
\hline J & 100 & 7.661 & 7.567 & 7.817 & 0.250 & $\mathrm{n}$ \\
$\mathrm{J}$ & 500 & 7.602 & 7.521 & 7.580 & 0.081 & $\mathrm{y}$ \\
$\mathrm{J}$ & 1000 & 7.576 & 7.592 & 7.549 & 0.043 & $\mathrm{n}$ \\
\hline JS & 100 & 8.320 & 7.916 & 8.016 & 0.404 & $\mathrm{n}$ \\
JS & 500 & 7.439 & 7.580 & 7.526 & 0.141 & $\mathrm{n}$ \\
JS & 1000 & 7.545 & 7.408 & 7.476 & 0.137 & $\mathrm{y}$ \\
JS & 2000 & 7.422 & 7.540 & 7.399 & 0.141 & $\mathrm{n}$ \\
\hline MJS & 500 & 8.999 & 8.712 & 8.756 & 0.187 & $\mathrm{n}$ \\
MJS & 1000 & 8.627 & 8.658 & 8.726 & 0.099 & $\mathrm{y}$ \\
MJS & 2000 & 8.785 & 8.720 & 8.704 & 0.081 & $\mathrm{n}$ \\
\hline EMJS & 500 & 7.855 & 10.31 & 8.874 & 2.452 & $\mathrm{n}$ \\
EMJS & 1000 & 7.648 & 7.751 & 7.661 & 0.103 & $\mathrm{n}$ \\
EMJS & 2000 & 7.607 & 7.619 & 7.686 & 0.079 & $\mathrm{y}$ \\
\hline VEMJS & 1000 & 9.441 & 8.520 & 9.474 & 0.954 & $\mathrm{n}$ \\
VEMJS & 2000 & 8.540 & 9.253 & 8.118 & 1.135 & $\mathrm{n}$ \\
VEMJS & 4000 & 8.057 & 7.929 & 7.921 & 0.136 & $\mathrm{y}$ \\
\hline
\end{tabular}

Table 9.1 The influence of population size on the Evolution Program in Galomusit. Note that the swing-by sequence only indicates the swing-by planets in between the launch from Earth and the capture at Neptune; e.g. 'JS' stands for an Earth-Jupiter-Saturn-Neptune sequence.

different population sizes. The $\Delta V$ of the best individual found after each run is indicated. The sequences indicate the flyby planet(s) during a trajectory from Earth to Neptune. This already gives a quick look on the final results; they will be discussed in much more detail in chapter 10. With this table the optimal population size can be determined for each number of flybys. The number of flybys is so important for the population size since it determines the size of the search space. Adding one flyby means multiplying the already available search space with many more possibilities. Thus the search space increases exponentially with the number of flybys. To enable EvP to still find the optimum the population size can be enlarged. This cannot be done without bound, since the computation time increases approximately linearly with population size. EvP is a partially-random method, and therefore the optimum will differ from run to run. But it should not differ too much, because that would indicate that the optimum is not found. This can be illustrated by the first case, using a single flyby past Jupiter. When taking a population of 100 individuals the difference between the "best best" individual and the "worst best" individual is $0.25 \mathrm{~km} / \mathrm{s}$. The next row indicates a significant decrease of the margin. Then taking a population size of 1000 the margin drops further, but it is deemed not sufficient to justify a doubling of the computation time. For these reasons a population size of 500 is chosen for the single-swing-by sequences. A similar approach has been used for the other sequences, sometimes also using the absolute values instead of only the relative values. Whether a certain population size is used for a certain number of swing-bys is indicated in the last column. The expected trend, namely a larger population for an increasing number of swing-by planets, can clearly be seen from these results. Since the found margins indicate that the stochastic nature of $\mathrm{EvP}$ is still present, it was decided that for each optimization in this thesis work three runs are executed. 


\subsection{Verification of Galomusit}

In this section the Galomusit tool will be verified. Firstly a comparison will be made with the older version of Galomusit as it was developed originally by R.M.H. Schlijper. This should show that no errors were made while adapting the program, or to put it more nicely: it should indicate the consistency of the program. For Schlijper's thesis work a flyby mission to Pluto was the objective. He found that in the 2005-2020 time frame, the mission sequence EJP is optimal. For these settings the current version of Galomusit was run, and the resulting dates and other values are indicated in table 9.2, together with the results obtained with the old version. It can be seen that there is a very good correspondence with respect to the first two dates and the launch delta- $\mathrm{V}$. And in both cases the delta- $\mathrm{V}$ required for the swing-by is negligibly small (note that an exact correspondence will never occur, due to the stochastic nature of the optimization procedure). The arrival dates however are approximately two months apart. This is not surprising, since no capture is involved in this mission, and thus the capture delta- $\mathrm{V}$ is not included in the optimization parameter. Therefore the optimization is relatively insensitive to the exact arrival date at Pluto. In any case, the found resemblance between the output of the original and that of the current version indicates the consistency of the program.

\begin{tabular}{|l|c|c|}
\hline & Old Galomusit & New Galomusit \\
\hline Launch $\Delta V[\mathrm{~km} / \mathrm{s}]$ & 5.644 & 5.643 \\
\hline Launch Date & $2005 / 01 / 01$ & $2005 / 01 / 01$ \\
\hline Swing-by Date & $2006 / 06 / 21$ & $2006 / 06 / 22$ \\
\hline Swing-by $\Delta V[\mathrm{~km} / \mathrm{s}]$ & 0.0003 & 0.004 \\
\hline Arrival Date & $2019 / 09 / 11$ & $2019 / 11 / 12$ \\
\hline
\end{tabular}

Table 9.2 Verification using the original version of the Galomusit tool. The test case is a flyby mission to Pluto, using Jupiter as a swing-by planet.

The verification would not be complete without comparing the results obtained by Galomusit with the output of another program. Therefore a comparison will be made with an example from the Software User Manual of the Swing-By Calculator (SBC) [JAQAR, 2005]. It comprises of a payload mass optimization for a spacecraft to Pluto, using aerogravity assists. In this way, the Lambert targeting procedure, the aerogravity assist implementation, and the optimization can all be verified at the same time. Though differences are likely to occur, since in the SBC it is optimized for mass using a particular launcher and specific impulse, while in Galomusit it is optimized for delta- $\mathrm{V}$ (only launch and swing-bys, not capture). They are almost one-to-one related, but it is not exactly the same. Furthermore, the SBC does not take into account the influence of height during an aerogravity assist (see section 6.4), and requires a particular height input (the used height at Venus of $80 \mathrm{~km}$ would result in a much too high density, but that is another discussion). In the SBC, an enumerative approach is taken, defining a date range for every planet involved, and going through this range first using a step-size of 5 days, and then refining to 1 day. In Galomusit only the launch date is constrained to be in 2013 (since the optimal launch date is found to be October 28, 2013 by the SBC), and the arrival to be before February 1, 2019 (in the SBC it is just set to the January 1, 2019). 


\begin{tabular}{|l|c|c|}
\hline & Galomusit & $S B C$ \\
\hline Launch $V_{\infty}[\mathrm{km} / \mathrm{s}]$ & 9.194 & 9.088 \\
\hline Launch Date & $2013 / 10 / 28$ & $2013 / 11 / 03$ \\
\hline Swing-by Date Venus & $2013 / 12 / 27$ & $2013 / 12 / 27$ \\
\hline Swing-by Date Mars & $2014 / 03 / 19$ & $2014 / 03 / 16$ \\
\hline Arrival Date & $2018 / 10 / 14$ & $2019 / 01 / 01$ \\
\hline Arrival $V_{\infty}[\mathrm{km} / \mathrm{s}]$ & 30.888 & 31.274 \\
\hline
\end{tabular}

Table 9.3 Verification using the results of the Swing-By Calculator for an aerogravity assist mission to Pluto.

Using these inputs, an optimal trajectory was found using Galomusit which can be compared with the SBC results. In table 9.3 some relevant dates and values are displayed. A good resemblance is found between these values. The date for the Venus gravity assist is even exactly the same. With respect to these swing-bys: the difference in the arrival and departure $C_{3}$ of the swing-bys falls in a predefined range of $5 \%$ in the SBC, and the delta-V's required for the swing-bys in Galomusit are only $3 \mathrm{~m} / \mathrm{s}$ and $0.5 \mathrm{~m} / \mathrm{s}$. Effectively both these results show an unpowered gravity assist. The date of arrival significantly differs, but this is due to the low sensitivity this date has on the optimization parameter, in which the capture delta$\mathrm{V}$ is not represented. This capture delta- $\mathrm{V}$ would be exceptionally high, looking at the arrival $V_{\infty}$ of more than $30 \mathrm{~km} / \mathrm{s}$. But this was not the intention of this optimization, which optimizes a flyby mission, not an orbiter mission. Looking forward to the intended results of this thesis for an orbiter mission to Neptune (which is also far away from the Sun, just like Pluto), these short flight times are not feasible due to the high arrival velocities. Concluding the verification, it can be stated that the found correspondence gives a good insurance of the reliability and correctness of the implementation in Galomusit. 


\section{Chapter 10}

\section{Results}

In this chapter the results of the trajectory calculations will be presented. The settings and boundary conditions for which these have been obtained will be discussed first in section 10.1. The trajectories obtained with chemical propulsion will be shown and discussed (section 10.2), which are naturally followed by the low-thrust trajectories (section 10.3). How these trajectories can be obtained became apparent in the foregoing matter of this thesis report. The emphasis is on presenting the resulting trajectories and analyzing their interesting nature.

\subsection{Settings and Boundary Conditions}

The objective of the optimization is to minimize the required energy. This is portrayed by the required delta- $\mathrm{V}$. In this delta- $\mathrm{V}$ the following components have been taken into account:

Launch: The delta-V to place the spacecraft in an Earth escape orbit, starting in a Geostationary Transfer Orbit, with a perigee height of $185 \mathrm{~km}$.

Swing-by: The delta-V(s) required for the powered swing-by(s), as was explained in section 6.3 .

Capture: The delta-V to place the spacecraft in a science orbit around Neptune, with periapsis and apoapsis height of 4000 and $48800 \mathrm{~km}$, respectively. This is to match Triton's orbit plane and motion [Noca and Bailey, 2004].

In order to also take the time of flight into account, minima and maxima were set on the launch and arrival date. The following rationale was used: to find a trajectory for an upcoming mission, the launch date cannot be set to tomorrow; some years should lie in between for developing, manufacturing and testing. Secondly, an interplanetary trajectory like this takes many years, see for example the Voyager missions [Carroll and Ostlie, 1996]. By not being too stringent, and to enable the optimizer to find feasible trajectories in terms of propellant, the maximum time of flight was set to 20 years in the first and 15 years in the second run. To conclude this, in the first run the first allowable launch date was set to January 1, 2010 and the maximum date of arrival on January 1, 2030. For the second run these are January 1 of 2013 and 2028. Obviously, these dates are somewhat arbitrary. A multi-objective optimizer, optimizing both time and energy requirements, could 
take away the need for this quite arbitrary setting of the second interval: it should find shorter flight times automatically, without directly imposing a shorter flight. This multi-objective optimization will therefore be part of the recommendations (see chapter 12).

The order in which the planets can be used for a swing-by are certainly not known beforehand. Therefore multiple swing-by sequences are optimized separately, after which the resulting trajectories are simply ranked according to their total required delta- $\mathrm{V}$. The sequences are generated based on the following constraints:

- Mercury is not used, because of its close proximity to the Sun, which would result in stringent heat shielding requirements. Mercury is also significantly in the wrong direction (inward instead of outward).

- Uranus is not used, since in the mentioned time frame, the position of Uranus is disadvantageous with respect to Neptune (see figure 10.1). Flying by Uranus and keeping the anti-clockwise direction would result in a far too long flight time, and in the unlikely case that its gravity field is strong enough to swing the spacecraft in a clockwise heliocentric trajectory, the velocity relative to Neptune would become undesirably high.

- A maximum of 5 gravity assists, since a boundary must be set to prevent an infinite number of sequences from occurring. It is a realistic maximum, since similar missions used 3 or 4 gravity assists [Melman, 2006].

- Only Venus and Earth can be used for consecutive flybys; Venus 3 times in a row, and the Earth 2 times. When using the other planets multiple times in the same sequence, the travel time would increase significantly due to their longer orbital periods.

From these constraints a total of 77 different swing-by sequences were found. These can be found in table 10.1. It is noted that the possibility to use Venus or Earth for a consecutive flyby increases the number of sequences significantly.
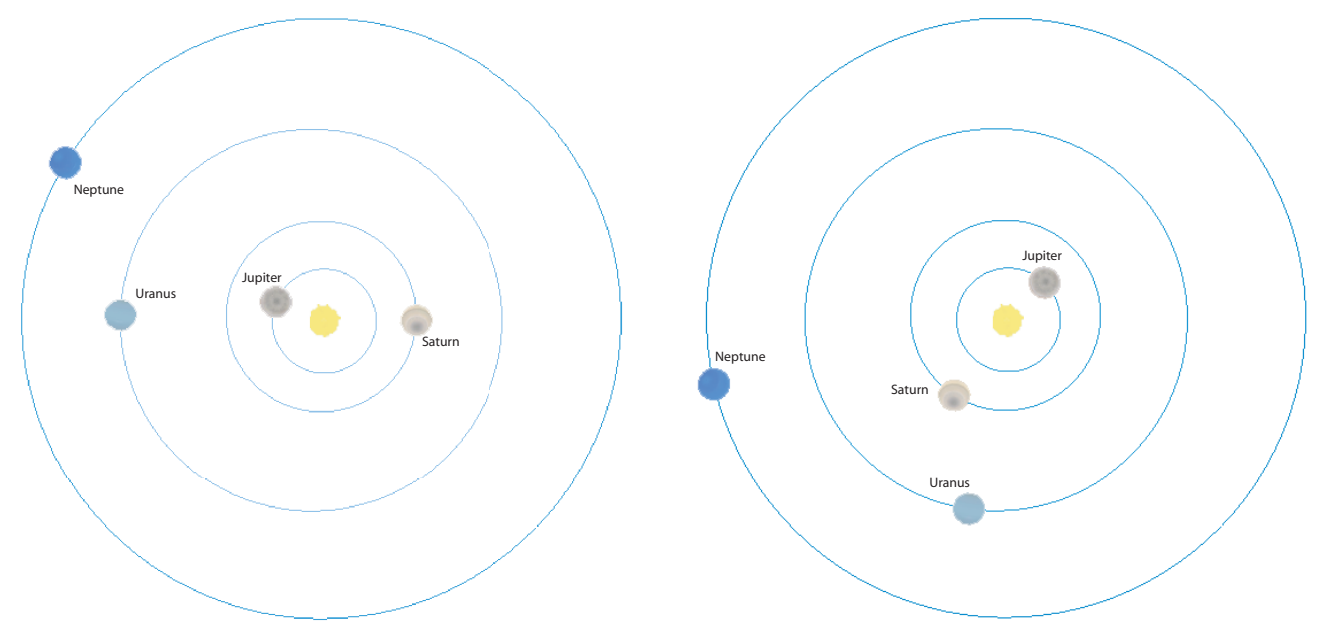

Figure 10.1 Position of the outer planets in 2010 (left) and 2030 (right). For esthetic reasons the planets and the Sun are all displayed at 10,000 times the relative size of Neptune. 


\begin{tabular}{|c|c|c|c|c|c|c|}
\hline V & E & M & J & S & VV & VE \\
\hline VM & VJ & VS & EE & EM & EJ & ES \\
\hline MJ & MS & JS & VVV & VVE & VVM & VVJ \\
\hline VVS & VEE & VEM & VEJ & VES & VMJ & VMS \\
\hline VJS & EEM & EEJ & EES & EMJ & EMS & EJS \\
\hline MJS & VVVE & VVVM & VVVJ & VVVS & VVEE & VVEM \\
\hline VVEJ & VVES & VVMJ & VVMS & VVJS & VEEM & VEEJ \\
\hline VEES & VEMJ & VEMS & VEJS & VMJS & EEMJ & EEMS \\
\hline EEJS & EMJS & VVVEE & VVVEM & VVVEJ & VVVES & VVVMJ \\
\hline VVVMS & VVVJS & VVEEM & VVEEJ & VVEES & VVEMJ & VVEMS \\
\hline VVEJS & VVMJS & VEEMJ & VEEMS & VEEJS & VEMJS & EEMJS \\
\hline
\end{tabular}

Table 10.1 77 Different sequences to reach Neptune. The start and end points are not mentioned, since they are the same for all (Earth and Neptune, respectively). From left to right, they are ordered on the number of flybys first and then from the inner solar system planets outwards.

\subsection{Results for High-Thrust Trajectories}

In chapters 6 and 9 it was explained how to obtain optimal high-thrust trajectories. Using the settings and boundary conditions from section 10.1, interesting and useful results can be obtained, which are point of discussion in this section. In fact four cases have been studied, with and without aerogravity assist, combined with the 2010-2030 time frame or the 2013-2028 time frame. For each of the 77 sequences in each case, three optimization runs are executed, as was already indicated in section 9.4. First the results of the pure gravity assist for the longer time of flight is shown in figure 10.2. From the results of all 77 sequences, only the best 20 are shown. A clear distinction is made between launch, swing-by and capture delta-V.

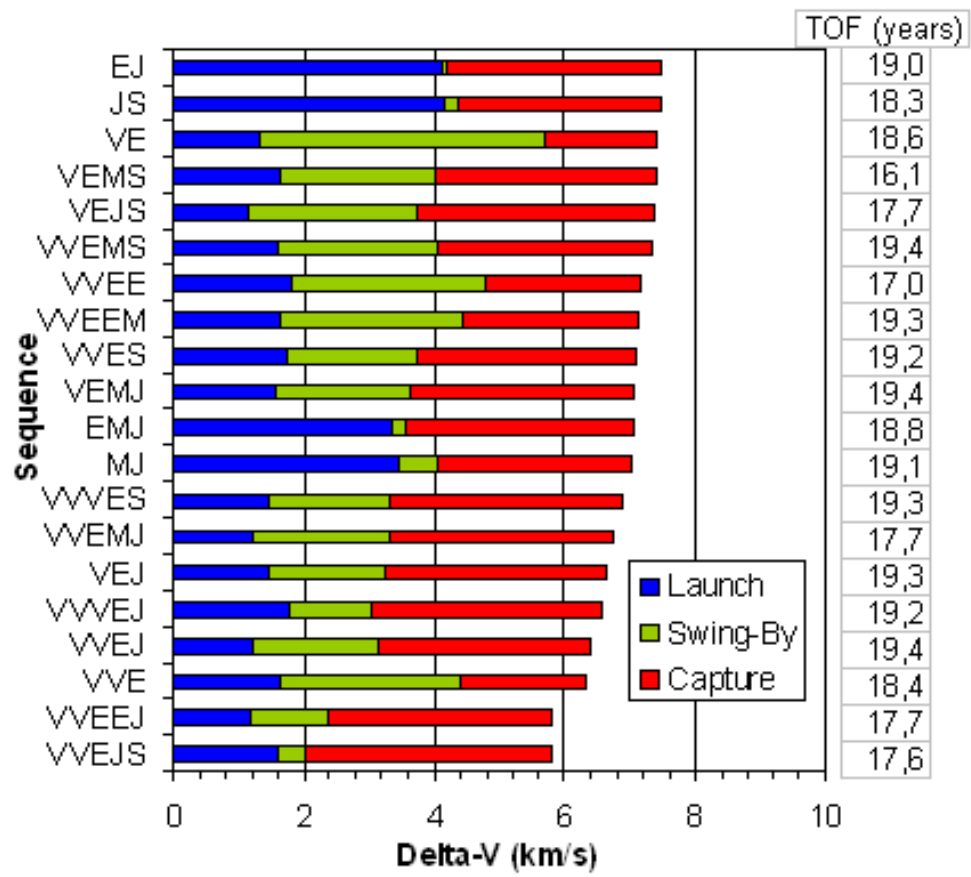

Figure 10.2 The 20 best sequences to Neptune in the 2010-2030 time frame, using pure gravity assists. 
Some interesting characteristics of swing-by trajectories can be derived from this figure. A clear tendency is observed towards multiple gravity assists, since no single gravity assist trajectory is in the top 20, and only 4 out of 12 dual gravity assist trajectories are present in these 20 best sequences. Further strengthening this observation is the fact that 5 out of the best 8 sequences comprise of a trajectory with 5 gravity assists. This tendency can certainly be explained, since more gravity assists means more possibilities to gain velocity for free, instead of gaining velocity by thrusting. On the other hand, more swing-bys also means more constraints, since these planets have to be encountered, irrespective of whether their relative geometry is advantageous. Even the extra degree of freedom, namely the swing-by date, cannot ensure that the disadvantage of an extra constraint can be neutralized. This explains why not all trajectories with 5 gravity assists are represented in the top. Another observation is the minor presence of Mars. This is to be expected since Mars is not a very 'powerful' swingby planet, due to its low mass and density. This was already shown in figure 3.7. Also Saturn is badly represented, which brings us to another observation: if Jupiter and Saturn are included, the capture delta- $\mathrm{V}$ is substantially higher than when the last swing-by planet is Venus, Earth or Mars. This is due to the significant velocity increase given by the gas giants on the last part of the trajectory. The spacecraft arrives at Neptune with a higher velocity, and thus a larger velocity change is required to put it into orbit around Neptune. The disadvantage from the trajectories not including Jupiter and Saturn is in general the higher swing-by delta- $\mathrm{V}$. A good example is the VE gravity assist option, which requires relatively large delta-V's of $3.01 \mathrm{~km} / \mathrm{s}$ at Venus and 1.37 $\mathrm{km} / \mathrm{s}$ at the Earth. This is caused by the large energy increase required to go directly from an orbit in the inner region of the solar system to a planet as far away as Neptune.

Another aspect is of course the associated time of flight. As mentioned before, the actual optimization did not account for this parameter directly, but by setting bounds on the dates it was included indirectly. The optimization tool just found the dates that represented the best trajectory in terms of delta-V. But as it turns out all resulting trajectories arrive at Neptune in 2029. In that way the last part of such a trajectory is slower when compared to a trajectory with an arrival in e.g. 2028. This results in a lower capture delta- $\mathrm{V}$, which explains the clear tendency towards this year of arrival. The launch date however can vary more, depending on the geometry of primarily the inner planets. But still all trajectories have a launch date in 2013 or earlier, although the option exists for finding a later launch date. Overall, the time of flight varies between 15.6 years and 19.7 years. To enable the finding of faster trajectories the bounds have been tightened, of which the results will be shown later in this section.

The best sequence for these conditions turns out to be a VVEJS gravity assist trajectory, as can be seen from figure 10.2, with a total delta-V of $5.819 \mathrm{~km} / \mathrm{s}$ and a time of flight of 17.6 years. In table 10.2 more information on this trajectory is given. The height indicates the periapsis height during a certain gravity assist. The gained delta- $\mathrm{V}$ denotes the absolute difference between the heliocentric velocity after and before the gravity assist. $V_{p}$ is the velocity at periapsis $(4000 \mathrm{~km})$ of the incoming hyperbola, with respect to Neptune. It follows directly from the mentioned $C_{3}$-value. The heights for the gravity assists at the gas giants are significantly higher, but still at this distance a gravity assist can be quite beneficial, as is evidenced by the large heliocentric velocity increases. The gravity assists can 


\begin{tabular}{|c|c|c|c|c|}
\hline Planet & Date & $\Delta V[\mathrm{~km} / \mathrm{s}]$ & \multicolumn{1}{|c}{$C_{3}\left[\mathrm{~km}^{2} / \mathrm{s}^{2}\right]$} & \multirow{2}{*}{} \\
\cline { 1 - 3 } Earth & $2012 / 05 / 25$ & 1.5972 & 18.940 & \\
\hline Planet & Date & Req. $\Delta V[\mathrm{~km} / \mathrm{s}]$ & Gained $\Delta V[\mathrm{~km} / \mathrm{s}]$ & Height $[\mathrm{km}]$ \\
\hline Venus & $2012 / 11 / 08$ & .0010 & 4.160 & 4705.2 \\
Venus & $2013 / 12 / 16$ & .0030 & 2.419 & 2236.0 \\
Earth & $2015 / 09 / 25$ & .0094 & 4.244 & 1540.8 \\
Jupiter & $2017 / 12 / 28$ & .4172 & 10.441 & 42895.2 \\
Saturn & $2021 / 06 / 14$ & .0081 & 3.900 & 660958.4 \\
\hline Planet & Date & $\Delta V[\mathrm{~km} / \mathrm{s}]$ & $C_{3}\left[\mathrm{~km}^{2} / \mathrm{s}^{2}\right]$ & $V_{p}[\mathrm{~km} / \mathrm{s}]$ \\
\hline Neptune & $2029 / 12 / 18$ & 3.7831 & 139.823 & 25.033 \\
\hline
\end{tabular}

Table 10.2 Quantification of the best pure gravity assist trajectory in the 2010-2030 time frame: VVEJS.

be considered unpowered, since the required delta-V's are in the order of a few meters per second. The exception is Jupiter, at which the swing-by requires a modest delta- $\mathrm{V}$ of $0.42 \mathrm{~km} / \mathrm{s}$. The trajectory is visualized by means of Celestia, an open-source 3D astronomy program. The data file, required for plotting in Celestia, has been created with the tool Space Trajectory Analysis (STA), which is the result of an educational software project started up by ESA [Schouten, 2006]. An overview of the trajectory can be found in figure 10.3, while a detail of the inner solar system part is presented in figure 10.4. After each swing-by the semi-major axis, and thus the associated energy level and velocity, becomes clearly higher. Especially after the Jupiter encounter the trajectory is significantly bent, which was to be expected from its high increase in heliocentric velocity. Interesting to notice is that the best found swing-by sequence is in fact the same as the one used by the Cassini mission $[N A S A / J P L, 2006 a]$. The primary difference is that Saturn is the final destination of the Cassini spacecraft, while the Neptune mission uses Saturn as a swing-by planet. The discussed VVEJS trajectory forms a good option for the actual trajectory to be chosen. But there are other options, for example to include an aerogravity assist. This option will now be discussed.

AGA Trajectories Giving the spacecraft the possibility to fly through an atmosphere and thereby improving the performance of a gravity assist, creates a whole new scala of opportunities. This can be seen when comparing figure 10.2 with figure 10.5, the last one representing missions including one or multiple aerogravity assists. The most striking difference is the absence of green in most bars, principally implying unpowered aerogravity assists. The increase of the maximum bending angle via an atmospheric flight (see section 6.4) is the reason for this difference. In contrast to the pure gravity assist trajectories, flying by Mars becomes a very attractive option, as is evidenced by figure 10.5. Instead of a maximum bending angle of a few tens of degrees in the case of a pure gravity assist at Mars (obtained by using equation (3.25)), the trajectory can be bent much more by also utilizing the atmosphere. In this way the inner planets can already be effective enough to send the spacecraft directly to Neptune. No intervenience of Jupiter or Saturn is needed, which further decreases the total delta- $\mathrm{V}$ by decreasing the capture delta-V. For this reason neither Jupiter nor Saturn are used until the $13^{\text {th }}$ best sequence.

It should be noted that not all planets in the sequences are used for an aerogravity assist. It is only implemented if a pure gravity assist is not sufficient to 


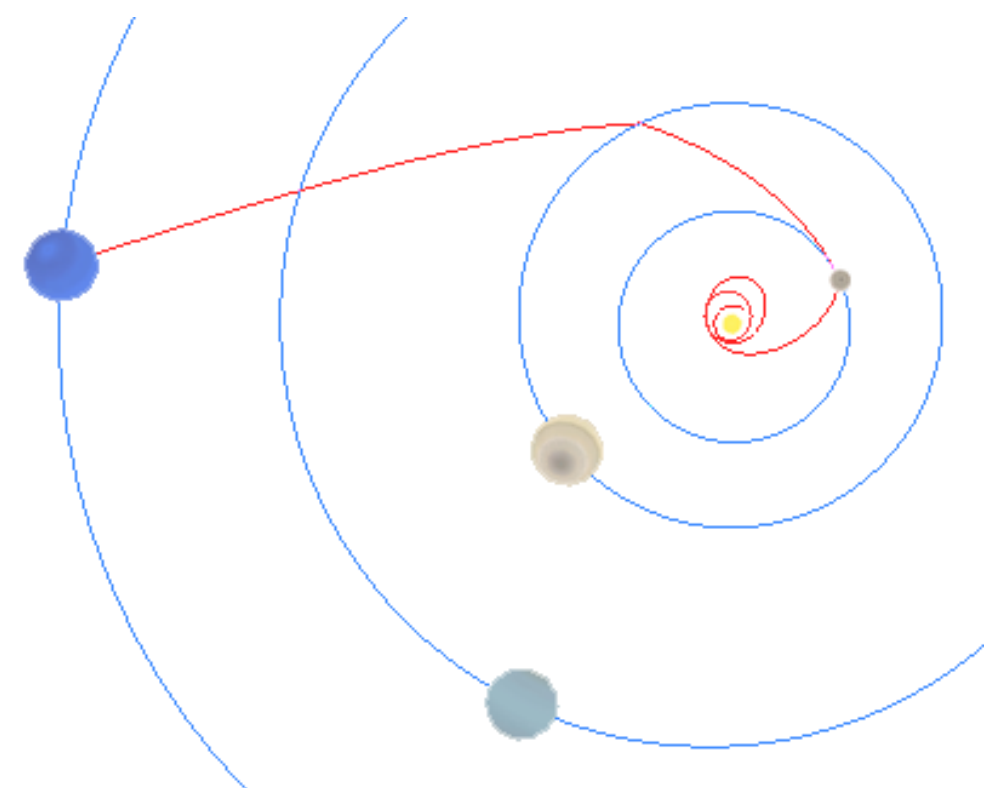

Figure 10.3 The best pure gravity assist trajectory: VVEJS. The positions of the outer planets are shown on the date of arrival at Neptune. For esthetic reasons the 3 outermost planets are displayed at 10,000 times the relative size of Neptune. The Sun and Jupiter are displayed smaller; otherwise they would block the trajectory.

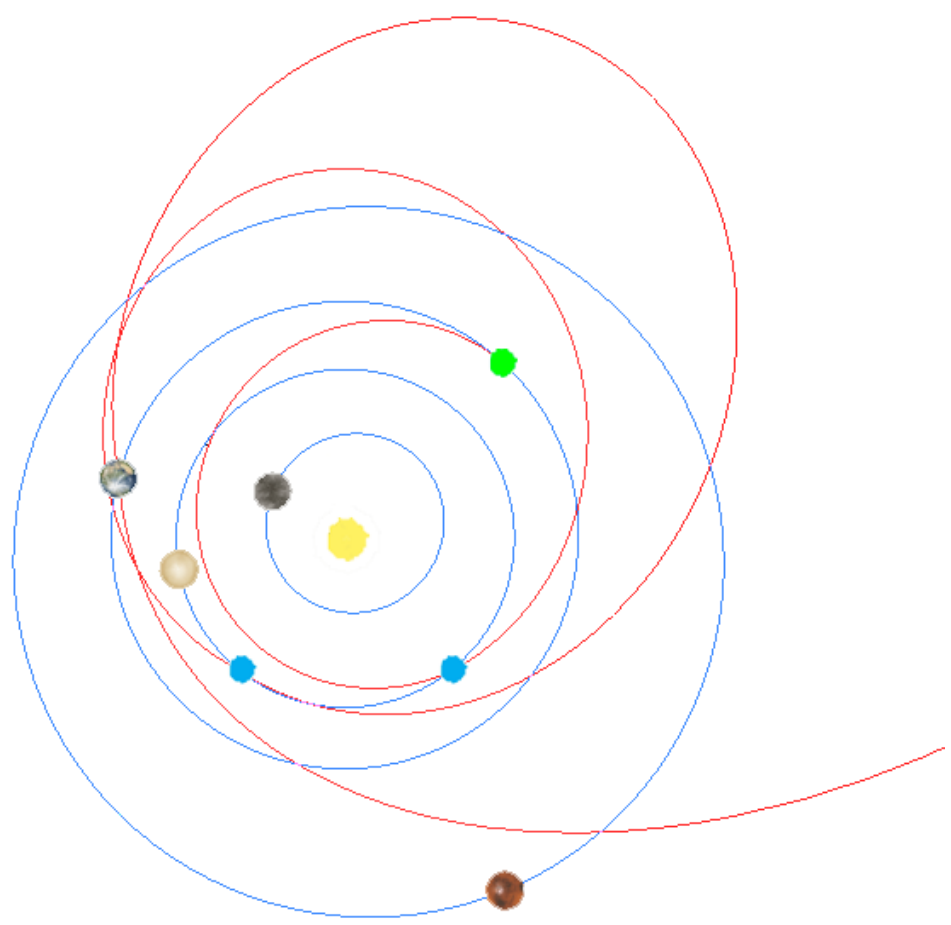

Figure 10.4 Detail of the best pure gravity assist trajectory: VVEJS. The positions of the planets are shown on the date of the swing-by at the Earth. The launch is indicated with a green dot and the two Venus swing-bys with a light blue dot. For esthetic reasons the planets and the Sun are displayed at 2,000 times the relative size of the Earth. 


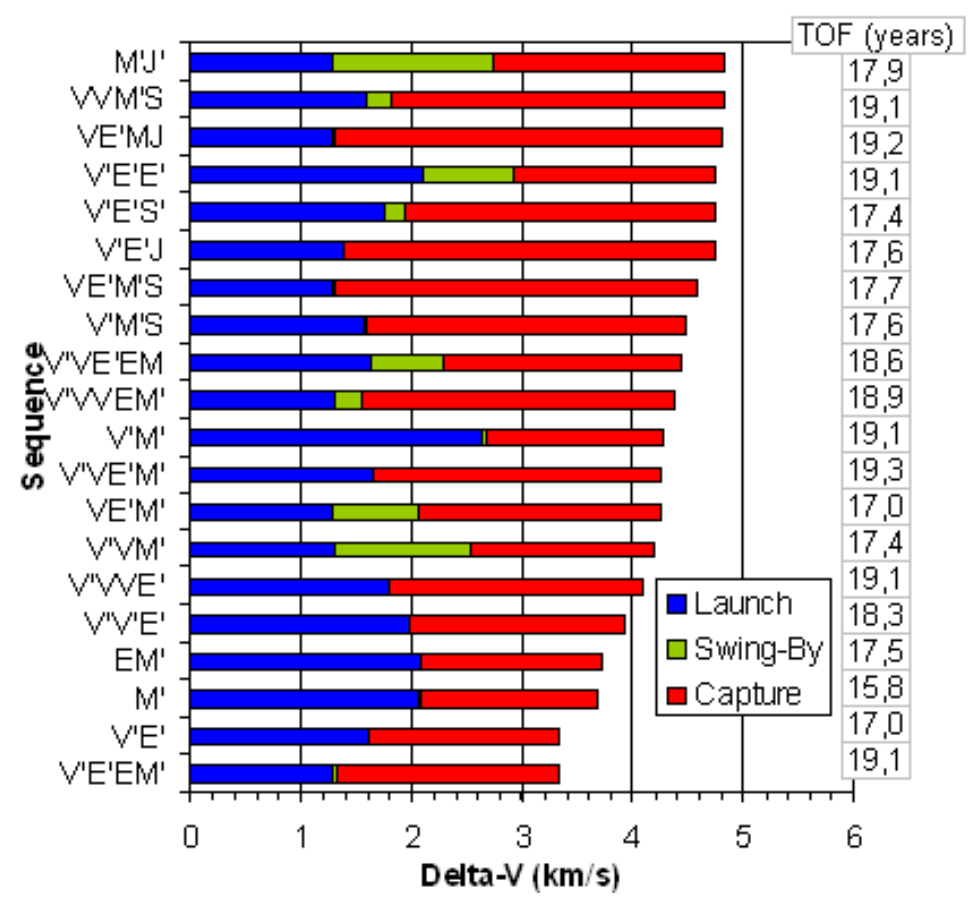

Figure 10.5 The 20 best sequences to Neptune in the 2010-2030 time frame, using aerogravity assists. A prime behind a planet identifier means that an aerogravity assist is performed during this particular flyby.

\begin{tabular}{|c|c|c|c|c|}
\hline Planet & Date & $\Delta V[\mathrm{~km} / \mathrm{s}]$ & \multicolumn{1}{|c}{$C_{3}\left[\mathrm{~km}^{2} / \mathrm{s}^{2}\right]$} & \multirow{2}{*}{} \\
\hline Earth & $2014 / 01 / 08$ & 2.0650 & 30.245 & \\
\hline Planet & Date & Req. $\Delta V[\mathrm{~km} / \mathrm{s}]$ & Gained $\Delta V[\mathrm{~km} / \mathrm{s}]$ & Height $[\mathrm{km}]$ \\
\hline Mars & $2014 / 05 / 19$ & .0135 & 8.727 & 73.8 \\
\hline Planet & Date & $\Delta V[\mathrm{~km} / \mathrm{s}]$ & $C_{3}\left[\mathrm{~km}^{2} / \mathrm{s}^{2}\right]$ & $V_{p}[\mathrm{~km} / \mathrm{s}]$ \\
\hline Neptune & $2029 / 11 / 08$ & 1.6080 & 45.653 & 22.858 \\
\hline
\end{tabular}

Table 10.3 Quantification of the best aerogravity assist trajectory in the 2010-2030 time frame: M.

patch the incoming with the outgoing hyperbolic leg. The planets at which in fact an AGA is performed, are indicated by a prime behind the planet identifier. Each trajectory at least one aerogravity assist is performed, from which it can be concluded that all trajectories with an AGA should perform better than without. A comparison between the two cases with and without an AGA confirms this. The best sequence including an AGA is the VEEM sequence. In fact, it is the best with respect to the required delta- $\mathrm{V}$, but as a serious disadvantage, it requires 3 aerogravity assists at 3 different planets. As was noted in section 6.4, this has some substantial sub-optimal effects, due to the different conditions in each atmosphere. Therefore it can be recommended that the number of aerogravity assist also forms part of the optimization. Although it is more luck than wisdom, the third best sequence only requires one atmospheric flight, and on top of that, it turns out to have the shortest time of flight of all the 77 sequences, namely 15.8 years. Therefore this will be considered the best option for the case including the AGA in the 2010-2030 time frame. Its characteristics are indicated in table 10.3. Although the launch delta- $\mathrm{V}$ is a bit higher than in the case of a pure gravity assist, the capture delta- $\mathrm{V}$ is substantially lower, resulting in a decrease of the total required delta- $\mathrm{V}$ of $2.133 \mathrm{~km} / \mathrm{s}$. This comes at the cost of making the spacecraft suitable for atmo- 


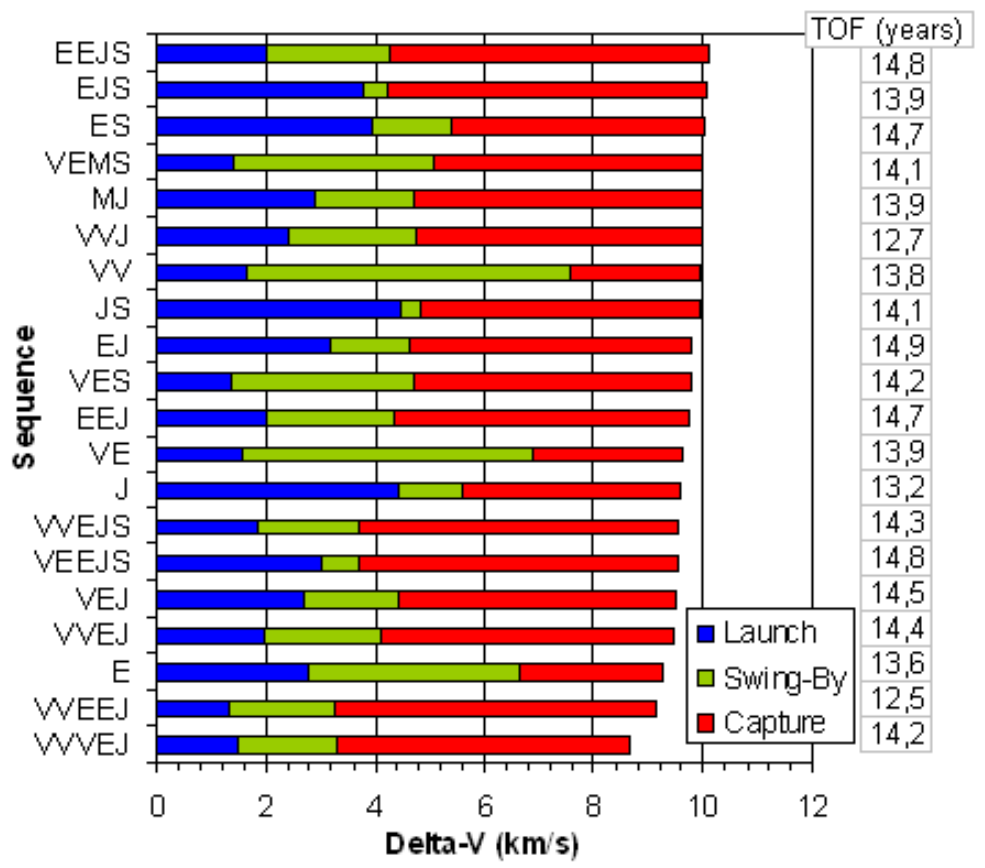

Figure 10.6 The 20 best sequences to Neptune in the 2013-2028 time frame, using pure gravity assists.

spheric flight, implying heat and radiation shielding. It might be the case that for the capture around Neptune, also an aero-assisted technique is used. This forms a part of a follow-up study. If the outcome of this research favors an aerocapture, it might be easier to implement an aerogravity assist at Mars, since in that case it is already designed for atmospheric flight. For the time being, no choice can be made between the best pure gravity assist trajectory and the best aerogravity assist trajectory, and both options will be presented to the person performing the aerocapture research.

Shorter Time of Flight As mentioned before, the time of flight can be reduced simply by putting the bounds on launch date and arrival date closer together. Both for the pure gravity assists and the aerogravity assists the bounds have been set on January 1 of 2013 and 2028. The resulting 20 best sequences are again plotted, this time in figures 10.6 and 10.7 respectively. What immediately becomes apparent, is that these faster trajectories require a higher delta-V. In fact, a comparison showed that this is the case for every sequence. This is to be expected, since faster trajectories imply higher velocities at whichever planet. Although this is not in general true, a faster trajectory could also have been found in the broader search space. This explains why in every case the tighter bounds perform worse in terms of delta- $\mathrm{V}$ than the looser bounds.

Also for the shorter flight times a closer look will be taken at the best solutions. In the case of pure gravity assists, the best trajectory from the 2010-2030 time frame (VVEJS) still performs well, but is now slightly surpassed by six similar trajectories. Only the swing-by trajectory via the Earth alone strongly differs. Although having a rather good total delta- $\mathrm{V}$, its swing-by delta- $\mathrm{V}$ is relatively large with respect to that delta- $\mathrm{V}$ of the other best solutions, since not one of the outer planets is used for a final swing-by. Also apparent from figure 10.6 is that 


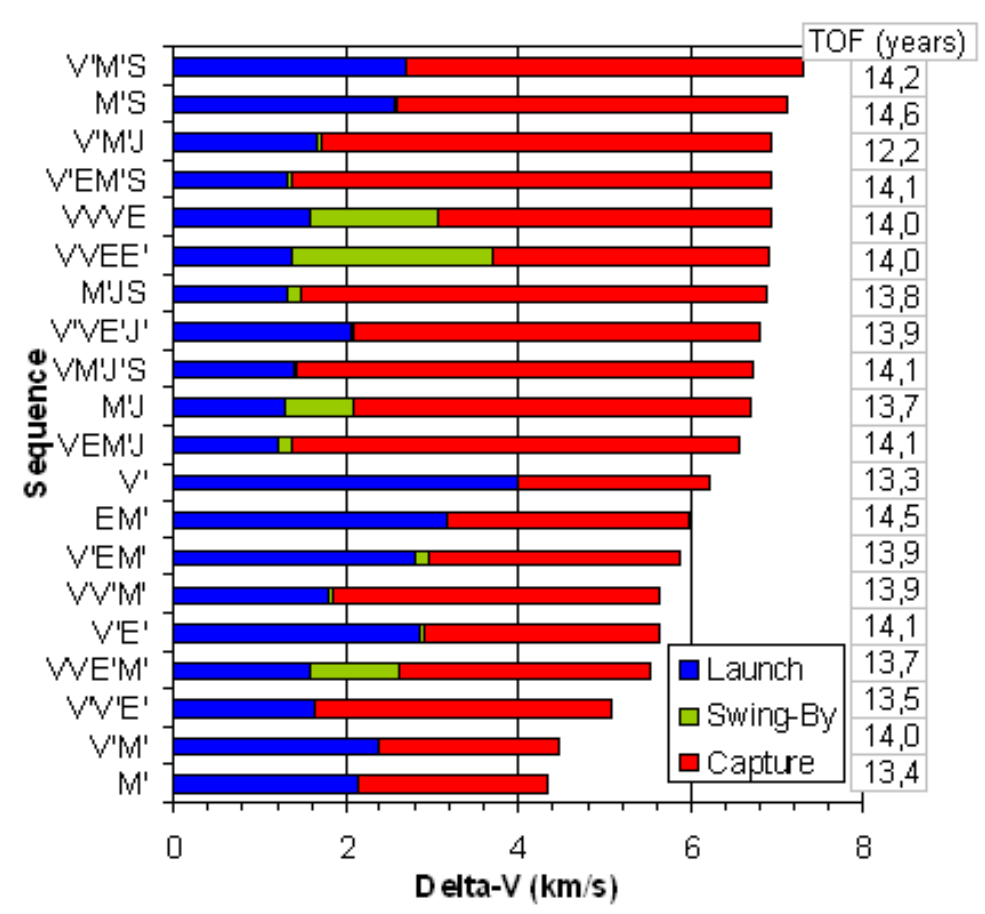

Figure 10.7 The 20 best sequences to Neptune in the 2013-2028 time frame, using aerogravity assists. A prime behind a planet identifier means that an aerogravity assist is performed during this particular flyby.

\begin{tabular}{|c|c|c|c|c|}
\hline Planet & Date & $\Delta V[\mathrm{~km} / \mathrm{s}]$ & \multicolumn{1}{|c}{$C_{3}\left[\mathrm{~km}^{2} / \mathrm{s}^{2}\right]$} & \multirow{2}{*}{} \\
\cline { 1 - 3 } Earth & $2013 / 10 / 28$ & 1.5013 & 16.677 & \\
\hline Planet & Date & Req. $\Delta V[\mathrm{~km} / \mathrm{s}]$ & Gained $\Delta \mathrm{V}[\mathrm{km} / \mathrm{s}]$ & Height $[\mathrm{km}]$ \\
\hline Venus & $2014 / 03 / 25$ & .0118 & 3.319 & 391.6 \\
Venus & $2015 / 05 / 29$ & .2726 & 0.018 & 328.7 \\
Venus & $2016 / 07 / 25$ & .0883 & 1.211 & 5091.4 \\
Earth & $2017 / 11 / 30$ & 1.1282 & 5.892 & 306.2 \\
Jupiter & $2019 / 10 / 07$ & .2840 & 13.380 & 159567.2 \\
\hline Planet & Date & $\Delta V[\mathrm{~km} / \mathrm{s}]$ & $C_{3}\left[\mathrm{~km}^{2} / \mathrm{s}^{2}\right]$ & $V_{p}[\mathrm{~km} / \mathrm{s}]$ \\
\hline Neptune & $2027 / 12 / 31$ & 5.3939 & 233.066 & 26.644 \\
\hline
\end{tabular}

Table 10.4 Quantification of the best pure gravity assist trajectory in the 2013-2028 time frame: VVVEJ.

the delta- $\mathrm{V}$ of the 20 best solutions does not vary much, making it difficult to really differentiate between the various sequences. However, the highest difference between two neighboring solutions is in fact between the best and the second best, namely a difference of $0.643 \mathrm{~km} / \mathrm{s}$. The best trajectory, VVVEJ, has a total delta$\mathrm{V}$ of $8.680 \mathrm{~m} / \mathrm{s}$ with a time of flight of 14.2 years. In table 10.4 more information on this trajectory is given. An important difference with the best trajectory of the longer time of flight is that the swing-bys are not practically unpowered. Especially the Earth swing-by requires a substantial delta-V. The second Venus swing-by seems not very useful, since it requires more delta- $\mathrm{V}$ than that is gained from it. But note that a swing-by is also used to change the orientation of the velocity. In this case this is apparently desired to obtain the right orbital geometry for yet another Venus swing-by. After this final Venus swing-by the spacecraft continues to Earth and Jupiter for very 'lucrative' swing-bys. Especially Jupiter contributes much, as is evidenced by the heliocentric velocity increase of $13.4 \mathrm{~km} / \mathrm{s}$. 
The capture at Neptune at the resulting high velocity, becomes quite problematic, as will be shown by the following discussion. In the case of a chemical capture, the required delta- $\mathrm{V}$ of $5.939 \mathrm{~km} / \mathrm{s}$ results in a propellant mass fraction of 0.849 (with an assumed specific impulse of $320 \mathrm{~s}$ ). So only $15 \%$ of the total mass of the spacecraft at arrival at Neptune would be available for constituents other than chemical propellants. In the case of an aerocapture, the thermal and structural constraints come into play. In the preparing work of my successor [Safipour, 2006], he found that these constraints cannot be satisfied if the velocity at infinity is above $12 \mathrm{~km} / \mathrm{s}$ (corresponding to an atmospheric entry velocity at $1400 \mathrm{~km}$ of $26 \mathrm{~km} / \mathrm{s}$ ). The velocity at infinity for the VVVEJ trajectory is $15.27 \mathrm{~km} / \mathrm{s}$, which seriously violates this constraint. Therefore no effort will be put in finding even shorter trajectories, as this will only result in even stronger violations of this constraint. The discussed VVEJS trajectory (of the 2010-2030 time frame) does in fact satisfy the mentioned constraint, having a velocity at infinity of $11.82 \mathrm{~km} / \mathrm{s}$. Therefore this VVEJS trajectory is regarded as the best found pure gravity assist trajectory.

\begin{tabular}{|c|c|c|c|c|}
\hline Planet & Date & $\Delta V[\mathrm{~km} / \mathrm{s}]$ & \multicolumn{1}{|c}{$C_{3}\left[\mathrm{~km}^{2} / \mathrm{s}^{2}\right]$} & \\
\hline Earth & $2014 / 01 / 07$ & 2.1449 & 32.222 & \\
\hline Planet & Date & Req. $\Delta V[\mathrm{~km} / \mathrm{s}]$ & Gained $\Delta V[\mathrm{~km} / \mathrm{s}]$ & Height $[\mathrm{km}]$ \\
\hline Mars & $2014 / 05 / 17$ & .0123 & 8.908 & 73.7 \\
\hline Planet & Date & $\Delta V[\mathrm{~km} / \mathrm{s}]$ & $C_{3}\left[\mathrm{~km}^{2} / \mathrm{s}^{2}\right]$ & $V_{p}[\mathrm{~km} / \mathrm{s}]$ \\
\hline Neptune & $2027 / 05 / 20$ & 2.1909 & 72.641 & 23.441 \\
\hline
\end{tabular}

Table 10.5 Quantification of the best aerogravity assist trajectory in the 2013-2028 time frame: M.

The discussion on the trajectories in the shorter time frame is now continued with a discussion on the AGA trajectories. From figure 10.7 conclusions can be drawn that are similar to the ones that were already discussed for the aerogravity assist trajectories in the longer time frame. Again the delta- $\mathrm{V}$ required for the swing-bys are in most of the cases effectively zero. This can be nicely illustrated with the VVEJ trajectory. Without AGA's it requires $2.15 \mathrm{~km} / \mathrm{s}$ to power the swing-bys, while with AGA's this is effectively reduced to zero. The delta-V for the launch and capture practically stays the same for both versions of the VVEJ trajectory. Furthermore, Mars still proves to be a planet that is very suitable for an AGA. This time, a single AGA at Mars is directly the best trajectory, without having to take into account the number of atmospheric entries. The characteristics of this trajectory can be found in table 10.5. A very strong resemblance can be noted with the longer AGA flight via Mars (see table 10.3). The launch date and the swing-by date only differ one and two days, respectively. The Mars-Neptune part, however, is considerably shorter. This results in a capture delta- $\mathrm{V}$ which is $0.583 \mathrm{~km} / \mathrm{s}$ higher. However, the velocity at infinity, being $8.52 \mathrm{~km} / \mathrm{s}$, is still below the constraint set for an aerocapture [Safipour, 2006]. So even faster trajectories could be searched for, which still satisfy this constraint. This has been done for the aerogravity assist trajectory via Mars, exclusively, as it displayed the best characteristics. Using Galomusit an optimal trajectory was found in a matter of seconds, for an arrival at Neptune before 2025. The exact characteristics are shown in table 10.6. The time of flight is now reduced to 10 years and 4 months, but at the cost of an increased capture delta- $\mathrm{V}$. However, with a velocity at infinity of $11.83 \mathrm{~km} / \mathrm{s}$ it is still possible to perform an aerocapture. Therefore this trajectory can be viewed as the best aerogravity assist trajectory, but as mentioned before, 
a further analysis of the aerocapture by my successor Ebrahim Safipour could further detail this conclusion. For his work on the capture he will require the exact orientation of the arrival velocity at Neptune. Therefore this velocity vector is given in table 10.7 for both the best pure GA and the best AGA trajectory.

\begin{tabular}{|c|c|c|c|c|}
\hline Planet & Date & $\Delta V[\mathrm{~km} / \mathrm{s}]$ & \multicolumn{1}{|c}{$C_{3}\left[\mathrm{~km}^{2} / \mathrm{s}^{2}\right]$} & \\
\hline Earth & $2014 / 01 / 14$ & 2.3397 & 37.088 & \\
\hline Planet & Date & Req. $\Delta V[\mathrm{~km} / \mathrm{s}]$ & Gained $\Delta V[\mathrm{~km} / \mathrm{s}]$ & Height $[\mathrm{km}]$ \\
\hline Mars & $2014 / 05 / 14$ & .0011 & 9.252 & 73.6 \\
\hline Planet & Date & $\Delta V[\mathrm{~km} / \mathrm{s}]$ & $C_{3}\left[\mathrm{~km}^{2} / \mathrm{s}^{2}\right]$ & $V_{p}[\mathrm{~km} / \mathrm{s}]$ \\
\hline Neptune & $2024 / 06 / 25$ & 3.5853 & 139.959 & 24.836 \\
\hline
\end{tabular}

Table 10.6 Quantification of the best aerogravity assist trajectory via Mars, with an imposed arrival before 2025 .

\begin{tabular}{|c|c|c|c|c|c|}
\hline Trajectory & Table & Arrival Date & $V_{X}[\mathrm{~km} / \mathrm{s}]$ & $V_{Y}[\mathrm{~km} / \mathrm{s}]$ & $V_{Z}[\mathrm{~km} / \mathrm{s}]$ \\
\hline VVEJS, pure GA & 10.2 & $2029 / 12 / 18$ & 11.1201 & 7.3877 & -0.3291 \\
\hline M, AGA & 10.6 & $2024 / 06 / 25$ & 11.2880 & 1.3606 & -0.2200 \\
\hline
\end{tabular}

Table 10.7 The heliocentric velocity of the spacecraft at Neptune for both the best pure GA and the best AGA trajectory. It is given in the J2000 ecliptic reference frame.

Mass As a final point, a rough comparison will be made between the mass delivered into orbit by both the best pure gravity assist trajectory (see table 10.2) and the best AGA trajectory (see table 10.6). It is limited to a rough comparison, since in this thesis no attention has been paid to the exact implications of an atmospheric flight on mass. As a basic assumption, the extra mass that is needed for an atmospheric flight (for shielding against heat input, for example) is taken to be one third of the total mass, as this was the case for the Huygens probe [ESA, $2006 a$ ]. It should be noted that this probe performed an atmospheric descent, which is obviously different from an AGA or aerocapture. But all these three maneuvers are atmospheric flights, by which a substantial change of the trajectory is obtained. Therefore they can be assumed to be roughly comparable with respect to mass requirements, and the mentioned assumption will suffice as a first-order approximation. Furthermore, the influence of an atmospheric flight through two different atmospheres is neglected, just as the dependence of the mass on the entry velocity.

The mass of the spacecraft after insertion into a hyperbolic escape trajectory is found by using the launcher performance, as it is described by equation 5.9. Partly due to the fact that the AGA trajectory is faster, the related $C_{3}$ at launch is higher than the one for the VVEJS trajectory. This results in a higher mass after insertion into a heliocentric orbit for the spacecraft following the VVEJS trajectory, namely $4706 \mathrm{~kg}$ versus $3490 \mathrm{~kg}$. Now a certain amount of this $4706 \mathrm{~kg}$ must be reserved for propellants to be able to perform the swing-bys of the VVEJS trajectory, which require a delta- $\mathrm{V}$ of $0.439 \mathrm{~km} / \mathrm{s}$. This results in a propellant mass fraction of 0.131 (assuming a specific impulse of $320 \mathrm{~s}$ ). Thus at the sphere of influence of Neptune the VVEJS trajectory delivers a spacecraft of $4092 \mathrm{~kg}$, while the trajectory via Mars delivers one of $2 / 3 \cdot 3490=2364 \mathrm{~kg}$ (in which the mass required for an atmospheric flight is taken into account). The last mentioned spacecraft needs no extra mass to perform a capture, since it is already capable of 
atmospheric flight. It is assumed that the spacecraft that has followed the VVEJS trajectory will perform a chemical capture at Neptune. This requires a substantial delta- $\mathrm{V}$ of $3.78 \mathrm{~km} / \mathrm{s}$, which results in a final mass in orbit around Neptune of $1226 \mathrm{~kg}$ (70\% of the mass is required for propellants). Summarizing, following the trajectory via Mars for an AGA, a spacecraft of $2364 \mathrm{~kg}$ can be put in an orbit around Neptune, while the spacecraft that follows the pure gravity assist trajectory weighs $1226 \mathrm{~kg}$ when finally captured at Neptune. Again it should be noted that this is based on the rough approximation that was made for the mass required for atmospheric flight. However, from these results can be concluded that the aero-assisted techniques are very promising, since using them can significantly increase the final mass of the spacecraft.

\subsection{Results for Low-Thrust Trajectories}

In chapter 7 it was explained how to calculate low-thrust trajectories using exponential sinusoids. These were implemented to find a trajectory to Neptune. A description of how this process took place will follow, culminating in a low-thrust trajectory to Neptune.

Firstly a qualitative discussion of the application of exponential sinusoids to a flight to Neptune will be given. It should be clear that multiple revolutions about the Sun on a direct trajectory to Neptune will result in an excessive time of flight, in the same order as the revolution period of Neptune about the Sun (165 years). This is certainly not desirable. On the other side of the spectrum of the time of flight there are the heliocentric escape trajectories; this is evidenced by for example the Saturn-Neptune leg in the high-thrust VVEJS trajectory (see table 10.2), which has a semi-major axis of -7.46 AU. These high-energy orbits are hard, if not impossible, to be modeled using exponential sinusoids. An escape trajectory, which is not periodic, cannot be simulated with exponential sinusoids, since these are intrinsically periodic. When velocities get close to or are higher than the heliocentric escape velocity (be it via a swing-by or a high launch $C_{3}$ ), the dynamics of the exponential sinusoid prescribe a deceleration of the spacecraft, to ensure a periodic orbit. This inability to follow a high-energy trajectory effectively (decreasing the velocity instead of increasing it) constitutes a shortcoming of the exponential sinusoid.

In the case of high-thrust trajectories, the trajectory is optimized using an evolution-based optimization method. For each individual (a set of dates at corresponding planets) the total required delta- $\mathrm{V}$ can be found, resulting in an easy-toobtain fitness value, which is needed for the correct functioning of the optimization method. However, in the case of exponential sinusoids, this becomes much harder, since for most of the initial conditions (launch date and hyperbolic excess velocity $V_{\infty}$ at launch) no actual encounters with the target planet can be found. Although a degree of freedom exists in choosing the right shape parameter $k_{2}$, in less than about $10 \%$ of the cases an actual encounter is found. This success rate decreases exponentially when swing-bys are included. This makes it hard to assign a fitness value to a particular individual, since most of them do not represent an actual trajectory from one planet to the other. Constraints could be built in, but this was not the chosen approach. Following the work by Petropoulos and Longuski [2004], an enumerative approach will be used. Over a range of launch dates and 
launch $V_{\infty}$ values will be searched for encounters with Neptune. From this broad search the most promising trajectory is selected and a refined search at a smaller step size is performed. This process will be shown for a trajectory directly from the Earth to Neptune, without implementing gravity assists. They were not included, because of practical time constraints set for a thesis research. Furthermore it is important to know that continuous thrusting will be applied throughout the low-thrust trajectory.

Hohmann In section 6.1 the so-called Hohmann transfer orbit was discussed. This orbit constitutes the minimum-energy, direct transfer orbit with chemical propulsion. The intention is now to surpass the performance of this trajectory by implementing electric propulsion. Firstly the time of flight of the resulting lowthrust trajectory should be shorter than 30.6 years (the flight time of a Hohmann trajectory). With respect to the required energy also a decrease is desired. In the case of high-thrust trajectories the energy was portrayed by the total required delta- $\mathrm{V}$ (see section 10.1). To simply add the delta- $\mathrm{V}$ required for the launch and the delta- $\mathrm{V}$ from the thrusting along the exponential sinusoid would be unfair with respect to the low-thrust propulsion, since the associated delta- $\mathrm{V}$ is applied with a much higher efficiency (higher specific impulse). Therefore the objective is changed to increasing the mass of the spacecraft delivered at Neptune. For a Hohmann trajectory to Neptune and using the performance data of the preferred launcher in this thesis work (see section 5.3), a spacecraft weighing $921 \mathrm{~kg}$ will arrive at Neptune (not inserted into orbit yet). To obtain a higher mass using a low-thrust technique, the launch $V_{\infty}$ should at least be smaller than the one required for a Hohmann trajectory $(11.25 \mathrm{~km} / \mathrm{s})$.

Broad Search For the earlier mentioned broad search, the launch $V_{\infty}$ is taken to be between 4.0 and $9.0 \mathrm{~km} / \mathrm{s}$, with a step size of $1.0 \mathrm{~km} / \mathrm{s}$. The earliest launch date is set to January 1, 2010, as it was argued for in section 2.4. The latest launch date is set to January 1, 2015. A step size of 10 days is taken. Using these settings a total of 124 exponential sinusoids have been obtained that reach Neptune. They are shown in figure 10.8 as a scatter plot of the propellant mass fraction (computed for an $I_{s p}$ of $3500 \mathrm{~s}$ ) against the launch date. It can be seen that in the last three months of each year opportunities exist to launch from the Earth and go to Neptune via an exponential sinusoid. It is also clear from this picture that a smaller launch $V_{\infty}$ results in most of the cases in a higher propellant mass fraction.

The launch $V_{\infty}$ and propellant mass fraction can now be combined to result in a spacecraft mass that can be delivered at Neptune. To determine the spacecraft mass after launch, the smallest mass is taken from the following two:

- The mass as prescribed by the launcher and the launch $V_{\infty}$.

- The mass that results from the maximum thrust divided by the maximum thrust acceleration $\left(m=T_{\max } / a_{\max }\right)$.

The maximum thrust acceleration follows from the dynamics of the exponential sinusoid, while the maximum thrust follows from the chosen engine specifications from section 5.2.3. It was found that a set of three engines is required (resulting in 


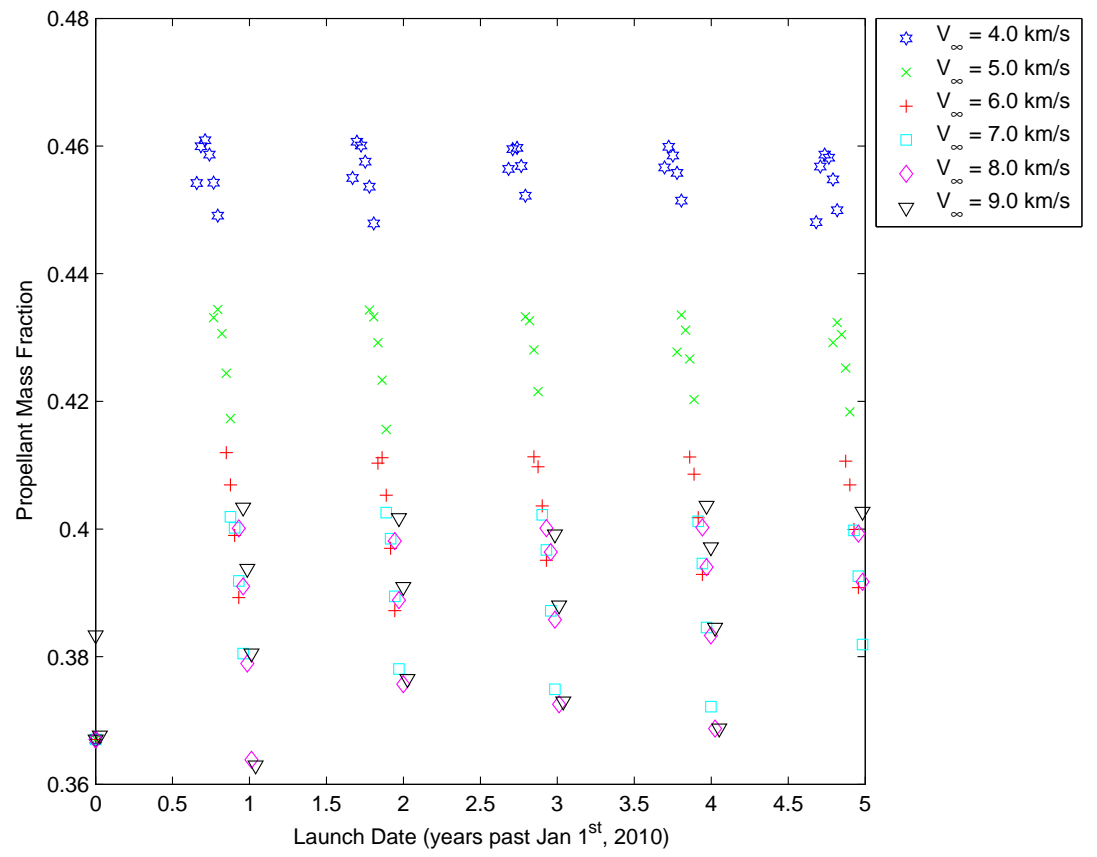

Figure 10.8 Propellant mass fraction against the launch date for various values of the launch $V_{\infty}$. It displays the results of a broad search for a low-thrust trajectory to Neptune.

a maximum available thrust of $0.75 \mathrm{~N}$ ) to obtain good performance when compared to the one of the Hohmann trajectory. This value for the number of thrusters is very reasonable, since in the design of the BepiColombo spacecraft five of these thrusters are implemented (one spare) [ESA-SCI, 2000]. The influence of the capture is left out of the calculation for the mass, since the arrival velocities at infinity were found to vary only slightly. For flight times below 30 years, the velocity $V_{\infty_{N}}$ is found to be between $3.5 \mathrm{~km} / \mathrm{s}$ and $4.5 \mathrm{~km} / \mathrm{s}$, whereas it is $4.05 \mathrm{~km} / \mathrm{s}$ for a Hohmann trajectory. These velocities are all well within the requirement set for an aerocapture, namely an infinite arrival velocity below $12 \mathrm{~km} / \mathrm{s}$ [Safipour, 2006]. These low arrival velocities can again be ascribed to the nature of the exponential sinusoids, namely not being able to simulate high-energy trajectories.

Using the mentioned approach for calculating the mass, another scatter plot was produced, relating the mass to the time of flight (see figure 10.9). Only trajectories with flight times that are shorter than the one for a Hohmann trajectory are shown. For a launch $V_{\infty}$ of $6.0 \mathrm{~km} / \mathrm{s}$ or lower, the mass after launch is governed by the maximum required thrust acceleration, and thus the available performance of the launcher cannot be fully employed. It is interesting to see that for $V_{\infty}$ equal to or above $7.0 \mathrm{~km} / \mathrm{s}$ (for which the launch mass is governed by the launcher and not by the maximum thrust) the mass at Neptune decreases for increasing time of flight. This indicates that for an exponential sinusoid the delta- $\mathrm{V}$ increases with time of flight (i.e. the average thrust acceleration decreases with increasing time of flight, but less than proportional). It makes the choice for an optimal trajectory rather easy, since for a specific launch $V_{\infty}$ the shortest time of flight corresponds to the highest mass. 


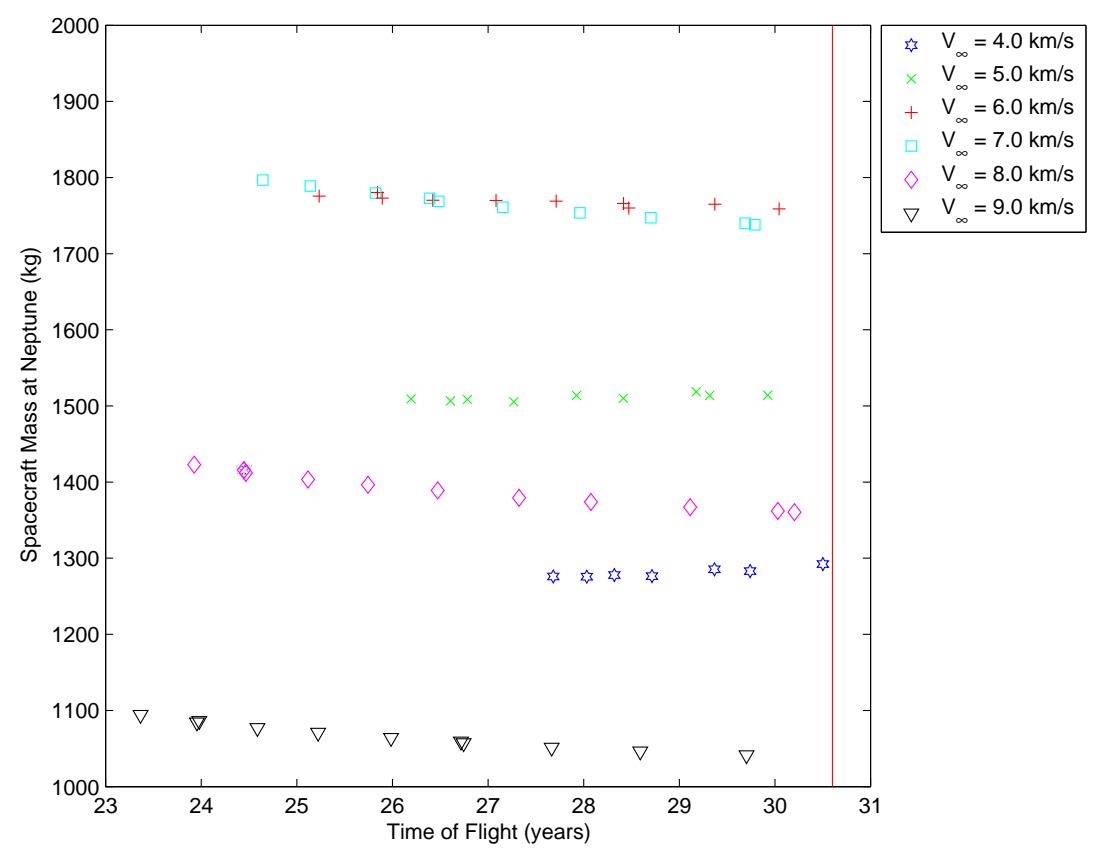

Figure 10.9 The mass delivered at Neptune (not inserted into orbit) against the time of flight for various values of the launch $V_{\infty}$. It displays the results of a broad search for a low-thrust trajectory to Neptune. The flight time related to a Hohmann trajectory is indicated as a red line.

Refined Search The significant increase in mass when comparing the best option with a launch $V_{\infty}$ of $7.0 \mathrm{~km} / \mathrm{s}$ with the best option with a launch $V_{\infty}$ of 9.0 $\mathrm{km} / \mathrm{s}$ is deemed to outweigh the small increase in time of flight. Therefore a refined search will be carried out for the best option with a launch $V_{\infty}$ of $7.0 \mathrm{~km} / \mathrm{s}$, having a launch on December 31, 2013. The launch date was allowed to vary around this date with a step size of one day. Since in general it can be stated that the best trajectories are found for a launch $V_{\infty}$ of $6.0 \mathrm{~km} / \mathrm{s}$ and $7.0 \mathrm{~km} / \mathrm{s}$, the launch $V_{\infty}$ is taken to be between those values, with a step size of $0.25 \mathrm{~km} / \mathrm{s}$. The relation between mass and time of flight is indicated in figure 10.10. All trajectories have a launch date in December 2013: December 31 turned out to be at the boundary of the launch date possibilities. It should be noted that similar results can be found for the December month of other launch years than 2013, as can be deduced from the repetitive pattern in figure 10.8 .

The refined search revealed trajectories with a better performance than the trajectories found from the broad search. Since the variation in flight time of the best options for each $V_{\infty}$ is very modest, again the choice is made for the highest deliverable mass: for a launch on December 25 with a launch $V_{\infty}$ of $6.5 \mathrm{~km} / \mathrm{s}$ a spacecraft weighing $1907 \mathrm{~kg}$ can be delivered at Neptune (in this figure the mass of the low-thrust propulsion system is still included). A plot of the associated trajectory is shown in figure 10.11. It can be seen that this trajectory is clearly different from a Hohmann trajectory, since that would be half an ellipse. From the indicated arrival date (September 13, 2038) it is clear that the objective to arrive at Neptune before 2030 is not met. As indicated before, this can be accredited to the inability of exponential sinusoids to follow a high-energy (and thus fast) trajectory. Another drawback of applying exponential sinusoids will become clear from the following discussion on the thrust acceleration profile. 


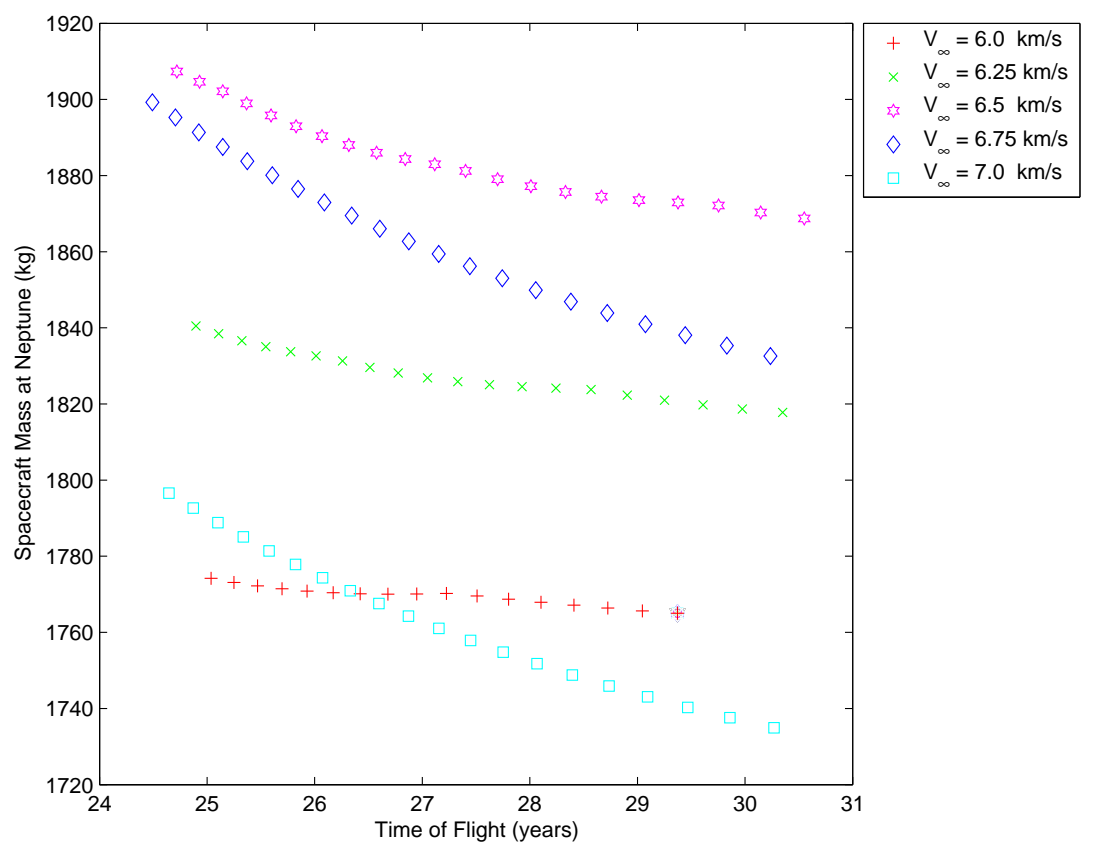

Figure 10.10 The mass delivered at Neptune (not inserted into orbit) against the time of flight for various values of the launch $V_{\infty}$. It displays the results of a refined search for a low-thrust trajectory to Neptune.

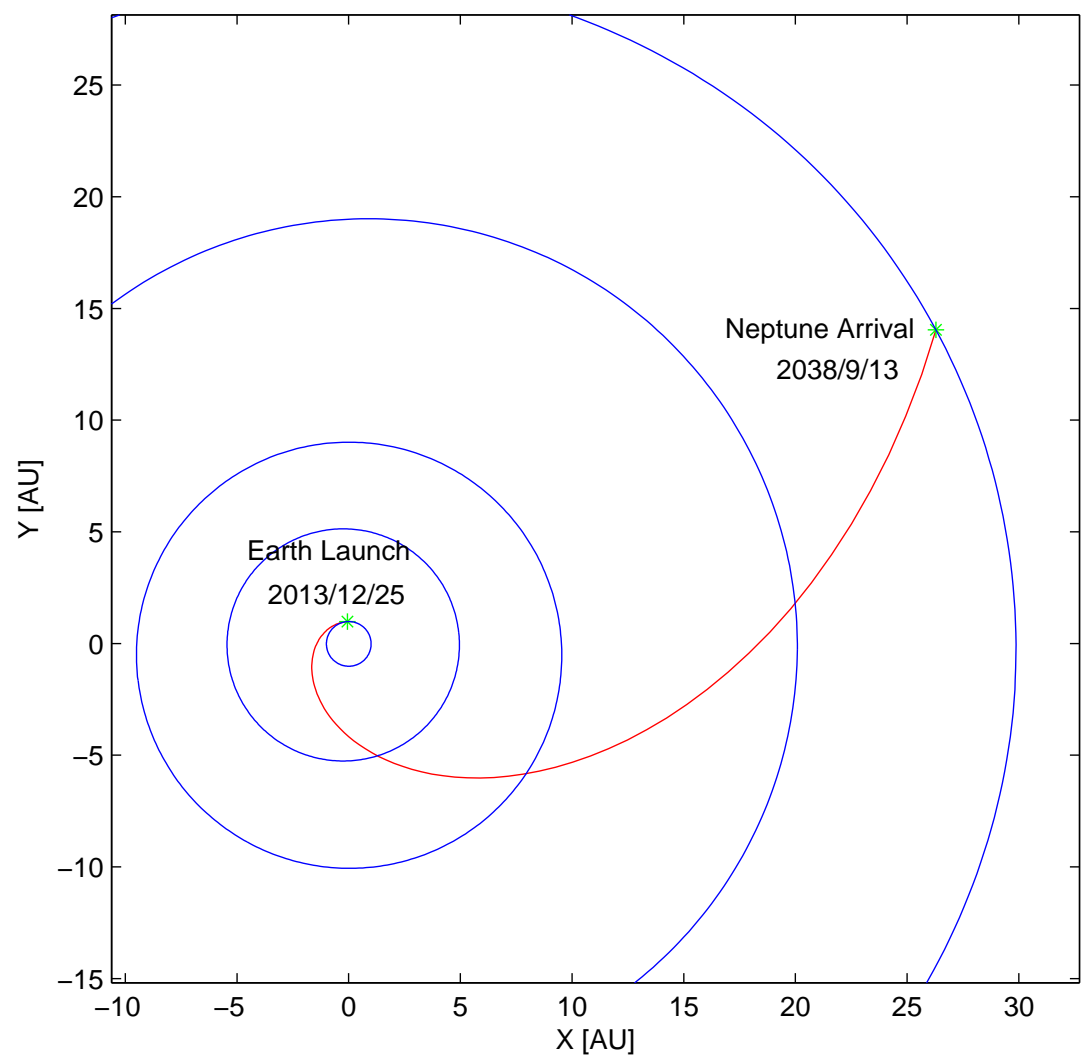

Figure 10.11 Ecliptic projection of the best found exponential sinusoid trajectory to Neptune. Launch takes place on December 25, 2013, with a $V_{\infty}$ of $6.5 \mathrm{~km} / \mathrm{s}$. The orbits of the Earth and the gas giants are indicated in blue. 


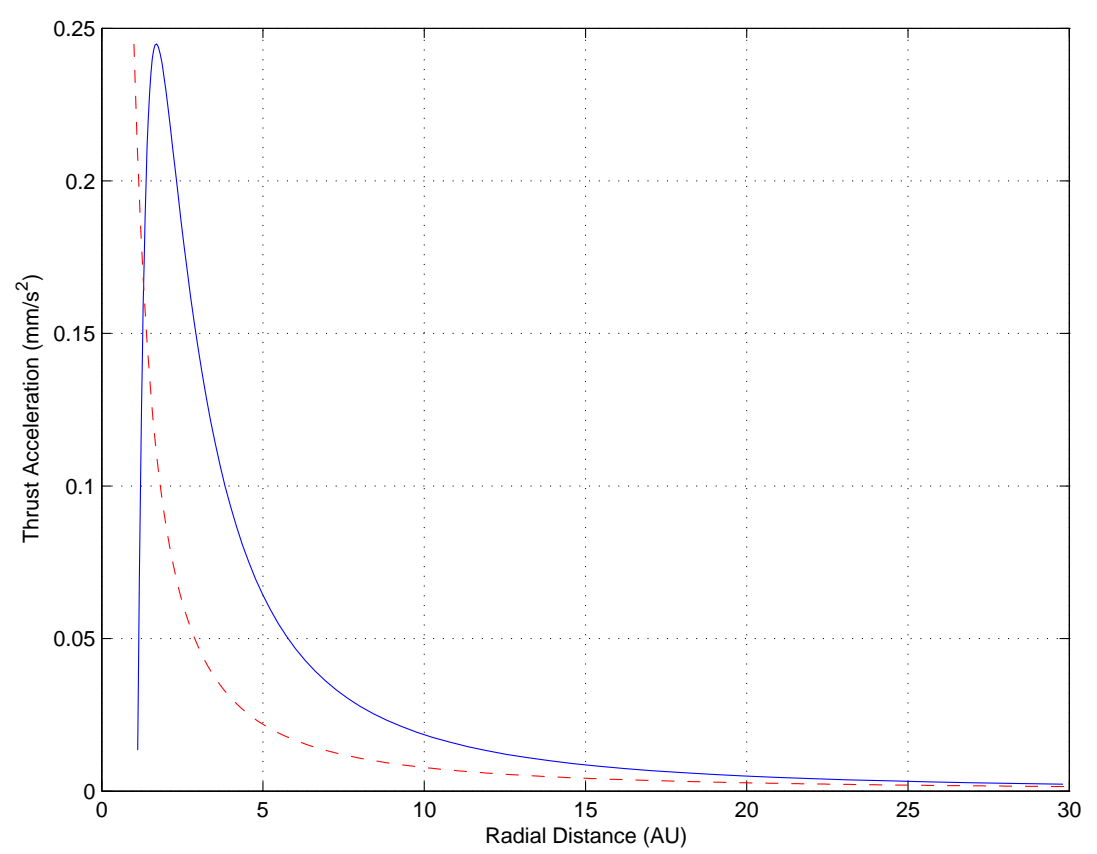

Figure 10.12 The thrust acceleration profile of the exponential sinusoid is indicated in blue. The available thrust acceleration is indicated by the red dashed line.

First it should be noted that the thrust acceleration that is going to be discussed is the in-plane acceleration. The out-of-plane acceleration was found to be negligible (as was already noted in section 7.2.1), with a maximum of $0.012 \mathrm{~mm} / \mathrm{s}^{2}$ and decreasing outwards, inversely proportional to the radial distance squared. However, the associated delta- $\mathrm{V}$ was taken into account when calculating the propellant mass fraction.

The thrust acceleration along the exponential sinusoid varies with radial distance as it is indicated by the blue line in figure 10.12. The maximum thrust acceleration $\left(0.2447 \mathrm{~mm} / \mathrm{s}^{2}\right)$ was taken into account in the calculation of the maximum mass. But it was assumed that this acceleration occurs at a radial distance of $1 \mathrm{AU}$. As it turns out, at this distance the thrust acceleration is approximately zero and it steadily increases outwards until the maximum is reached at a radial distance of $1.70 \mathrm{AU}$. The available thrust acceleration (as it follows from a thrust law with an exponent of 1.5 (see section 5.2.3)) is indicated in red, and is shortly after launch already lower than the required thrust acceleration to follow the exponential sinusoid. At a radial distance of about 3.37 AU the required thrust is more than three times higher than the available thrust. So even a doubling of the available thrust cannot accommodate a feasible profile, and would primarily result in a substantial surplus of thrust in the first part of the trajectory. The thrust acceleration profiles of the other exponential sinusoid trajectories were examined as well, and they showed the same behavior. Thus the profile that follows from the dynamics of an exponential sinusoid does not resemble an actual, realistic profile. Although the associated thrust acceleration levels are of the same order of magnitude as realistic values for the thrust acceleration, the associated profile does not conform well to reality. This forms another drawback of the exponential sinusoid.

Although the found flight time is rather long and the thrust profile does not have the desirable characteristics, it can be prudently concluded that continuous 
thrusting can have advantages above the high-thrust option, since shorter flight times and higher masses were found for low-thrust trajectories when compared to the performance of a Hohmann trajectory. No real comparison can be made between the results for the high-thrust trajectories from section 10.2 and the lowthrust trajectories, since the low-thrust trajectories did not implement gravity assists. Including those would be a valuable continuation of the research, in order to find better performing low-thrust trajectories. 
Chapter 11

\section{Conclusion}

The primary objective of this thesis is to explore possibilities for a mission to Neptune, the trajectory towards Neptune in particular. The interplanetary trajectory towards this planet is optimized for the minimum required energy, while the flight time is taken into account as well. The arrival at Neptune has to take place before 2030. Various techniques were investigated: (aero)gravity assists in combination with chemical engines and low-thrust techniques.

Concerning the results obtained for the high-thrust trajectories (chemical engines), the best two missions have been identified: one implementing pure gravity assists and the other one conducting aerogravity assists. A possible aerocapture was taken into account by choosing the best performing mission with an atmospheric arrival velocity below $26 \mathrm{~km} / \mathrm{s}$ (otherwise structural and thermal constraints cannot be satisfied). The two resulting trajectories are:

- Using pure gravity assists, a swing-by trajectory via Venus, again Venus, Earth, Jupiter, and Saturn shows the best performance. Launch takes place in May 2012 and the spacecraft arrives at Neptune in December 2029. Assuming that a chemical capture is performed, the spacecraft mass will be $1226 \mathrm{~kg}$ when finally in orbit around Neptune.

- Using aerogravity assists, a trajectory via the atmosphere of Mars is identified as the best. Launch takes place in January 2014 and the spacecraft arrives at Neptune in June 2024. Assuming an aerocapture at Neptune, the final spacecraft mass will be $2364 \mathrm{~kg}$ (without aerocapture system). This is an approximate figure, since no detailed research has been performed to find the exact implications of an atmospheric flight on the spacecraft's mass.

The shorter flight time and higher mass for the optimal aerogravity assist trajectory might suggest that this trajectory is the best. However, this technique has not been applied in practice before, while a pure gravity assist has been used extensively. For the time being, no definitive choice can be made between these two trajectories. Therefore both options will be presented to the person performing the capture research.

The low-thrust trajectories have been modeled analytically using so-called exponential sinusoids. A direct (i.e. without gravity assists) low-thrust trajectory from the Earth to Neptune shows better performance than the minimum-energy, direct transfer orbit with chemical propulsion (Hohmann trajectory). The flight 
time is reduced from 30.6 years to 24.7 years, while the mass delivered at Neptune (not inserted into orbit) is increased from $921 \mathrm{~kg}$ to $1907 \mathrm{~kg}$. However, the thrust profile related to the exponential sinusoid does not resemble completely an actual, realistic profile. Initially it increases outwards, instead of decreasing with increasing distance from the Sun. This is inherent to the analytical description of an exponential sinusoid. Another limitation of the exponential sinusoids is the inability to follow a high-energy trajectory. This is one of the reasons for not finding faster trajectories. A second reason is the absence of gravity assists in the low-thrust trajectory. Concluding the discussion on the low-thrust trajectories, no feasible trajectory has been found. However, Neptune can be successfully reached via the high-thrust trajectories.

The discussed results have been obtained using Galomusit, a software tool that was developed at the Faculty of Aerospace Engineering, Delft, by several MSc students. To make the program suitable for optimizing the flight to Neptune in particular, while at the same time making the program more universally applicable, numerous adaptations were successfully implemented:

- It can now deal with a flight from any planet in the solar system to any other one.

- It is made possible to include a capture at the destination planet.

- It can optimize multiple different swing-by sequences automatically, instead of one at a time.

- It is made possible to use a planet for two consecutive gravity assists.

- It is more user-friendly, since many elucidating comments have been added to the source code and all input can be specified in one single subroutine.

- It is much faster than before, because a more efficient way of generating possible solutions is implemented, using problem-specific knowledge. Computation times of 12 hours have been reduced to 10 minutes. 


\section{Chapter 12}

\section{Recommendations}

For this thesis work much research has been done, but several interesting areas remain to be investigated further. This results in multiple recommendations that will be described in this chapter. First of all, recommendations can be made regarding the modeling of the spacecraft trajectory:

- The assumption of the two-body problem enabled the use of Lambert targeting and exponential sinusoids, but the third-body forces and other perturbations (see chapter 4) were neglected. For preliminary mission studies this assumption suffices. It provides a good indication of the feasibility regarding the spacecraft trajectory. In more detailed phases of mission design, however, the necessity arises for high-accuracy trajectory determination. To obtain the required accuracy in these phases the perturbing forces also need to be incorporated, resulting in a full dynamic model, which can be numerically integrated and optimized.

- While modeling an aerogravity assist, the values for certain aerodynamic parameters need to be assumed, namely the lift-to-drag ratio $L / D$ and the wingload $\frac{m}{C_{L} A}$. Research can be done into the sensitivity of these parameters, in order to define their exact influence on the performance of an aerogravity assist.

- Also regarding these aerogravity assists, the implications of a flight through an atmosphere can be quantified, with respect to for example heat load and radiation exposure. Additionally it can be investigated what the actual suboptimal effects are by going through multiple different atmospheres at different heights with differing velocities, which implies a varying optimal design.

- The low-thrust trajectories are modeled by exponential sinusoids. In the way it is implemented by Petropoulos and Longuski [2004] and myself, it fails to make a direct connection with the Lambert targeting approach, as it is used for highthrust trajectories. It is in fact possible to find an exponential sinusoid from one point in space to the other in a given fixed amount of time (as is the case for Lambert targeting), as was shown by Izzo [2006]. This approach makes the undesirable out-of-plane motion and the cumbersome root-finding redundant (see sections 7.2.1 and 7.3.1, respectively). It will prove valuable to implement the Lambert analogy for the exponential sinusoids.

- The thrust profiles related to the exponential sinusoids (including the tangential thrust assumption) do not cover the full range of possibilities, and do 
not directly implement information about the thrusters. Therefore analytical methods other than the exponential sinusoid approach can be studied. A possibility would be to implement the method developed by Biesbroek [2006], which does use pre-described thruster models.

- As a last point on the low-thrust trajectories: a combination with gravity assists will enable finding faster trajectories, such that the requirement on the flight time can be met. Additionally, in this way it is possible to make a fair comparison between high-thrust and low-thrust trajectories.

Regarding the optimization the following recommendations can be given:

- The used optimization procedure is able to come close to the global optimum. This could be used as a starting point for a local optimization, implementing a full dynamic model, as was mentioned earlier in the recommendations.

- A single-objective optimization approach has been applied, minimizing the required energy. It will prove useful to extend this to a multi-objective optimization, also including the time of flight as an objective.

Additionaly, recommendations can be made with respect to the Galomusit tool:

- At the moment, Galomusit is capable of 'one-way traffic' only. In the case of a manned or a sample return mission, the return flight should be simulated and optimized as well. This would be a valuable extension of Galomusit. Also other functionalities could be thought of, such as the opportunity to enter a heliocentric orbit as a final condition.

- The mission layout and other parameters can easily be defined in a specific subroutine. The status of the optimization is shown while executing the program in a console window. A more user-friendly approach would be to develop a Graphical User Interface (GUI), which combines these functionalities.

- Galomusit can be unified with Optidus (OPTimization of Interplanetary trajectories by Delft University Students), which is another program developed by students at the Faculty of Aerospace Engineering. It uses numerical propagation techniques to simulate interplanetary trajectories, whereas Galomusit is more analytically-based. A modular approach should be strived for, such that the desired optimization procedure or simulation method can easily be selected.

Finally, recommendations can be made about the obtained results. A large search space has been effectively explored: many different swing-by sequences have been taken into account. But this search was still not exhaustive. For example, trajectories with more than 5 swing-bys could be investigated, as the use of multiple swing-bys showed good results. For the continuation of the design a closer look should be taken at the mass budgets, as these can be further refined. Especially the implications on mass of having an atmospheric flight and/or a large solar array for the electric propulsion require further study. 


\section{Bibliography}

Anderson Jr., J.D., M.J. Lewis, A.P. Kothari, and S. Corda (1991), Hypersonic Waveriders for Planetary Atmospheres, Journal of Spacecraft and Rockets, 28(4), p. 401-410, July - August 1991.

Battin, R.H., and R.M. Vaughan (1983), An Elegant Lambert Algorithm, Journal of Guidance, Vol. 7, No. 6, p. 662-670.

Biesbroek, G.J.R. (2006), A Simple Low-Thrust Lambert Solver Using PreDescribed Engine Models, JAQAR Internal Report, JAQ-SBC-PAP2006-LTT.

Biesbroek, G.J.R., and B.P. Ancarola (2003), Study of Genetic Algorithm Settings for Trajectory Optimisation, 54th International Astronautical Congress, IAC03-A.P.30.

Breakwell, J.V., and H.E. Rauch (1966), Optimum Guidance for a Low Thrust Interplanetary Vehicle, AIAA Journal, p. 693-704, April 1966.

Burden, R.L., and J.D. Faires (2001), Numerical Analysis, Pacific Grove: Brooks/Cole, 7th edition.

Carroll, W., and A. Ostlie (1996), An Introduction to Modern Astrophysics, Reading: Addison-Wesley.

Cornelisse, J.W., H.F.R. Schöyer, and K.F. Wakker (1979), Rocket Propulsion and Spaceflight Dynamics, London: Pitman Publishing Limited.

ESA (2006a), Huygens, Science and Technology, http://huygens.esa.int, Last accessed: April 20, 2006.

ESA (2006b), Rosetta, Science and Technology, http://rosetta.esa.int, Last accessed: July 6, 2006.

ESA (2006c), SMART-1, Science and Technology, http://smart.esa.int, Last accessed: April 10, 2006.

ESA-SCI (2000), BepiColombo, System and Technology Study Report, European Space Agency, Science Department.

Fortescue, P., J. Stark, and G. Swinerd (2003), Spacecraft Systems Engineering, Chichester: J. Wiley and Sons Ltd., 3rd edition.

Garot, D. (2005), Trajectory Optimization of a Solar Polar Sail Mission, Literature Report, Delft University of Technology, Faculty of Aerospace Engineering.

Goldberg, D.E. (1989), Genetic Algorithms in Search, Optimization, and Machine Learning, Reading: Addison-Wesley.

Gooding, R.H. (1990), A Procedure for the Solution of Lambert's Orbital Boundary-Value Problem, Celestial Mechanics and Dynamical Astronomy, Vol. 48, p. $145-165$. 
Izzo, D. (2006), Lambert's Problem for Exponential Sinusoids, Advanced Concepts Team, European Space Research and Technology Centre.

Jakobsen, T. (2004), Genetic Algorithms, http://subsimple.com/genealgo .asp, Last accessed: April 19, 2006.

JAQAR (2005), Swing-by Calculator - Software User Manual, JAQAR Space Engineering, Issue 7.3.

Larson, W.J., and J.R. Wertz (1999), Space Mission Analysis and Design, Torrance: Microcosm Press, 3rd edition.

Laursen, E.F. (2004), Proton Launch System Mission Planner's Guide, International Launch Services, Revision 6.

Lewis, M.J., and A.D. McRonald (1992), Design of Hypersonic Waveriders for Aeroassisted Interplanetary Trajectories, Journal of Spacecraft and Rockets, 29(5), p. 653-660, September - October 1992.

McConaghy, T.T., T.J. Debban, A.E. Petropoulos, and J.M. Longuski (2003), Design and Optimization of Low Thrust Trajectories with Gravity Assist, Journal of Spacecraft and Rockets, Vol. 40, No. 3, p. 380-387, May-June 2003.

McRonald, A.D., and J.E. Randolph (1992), Solar System "Fast Mission" Trajectories Using Aero-Gravity Assist, Journal of Spacecraft and Rockets, 29(2), p. 223-232, March - April 1992.

Melman, J.C.P. (2006), Trajectory Optimization for a Mission to Neptune and Triton, Literature Report, Delft University of Technology, Faculty of Aerospace Engineering.

Michalewicz, Z. (1996), Genetic Algorithms + Data Structures = Evolution Programs, Berlin: Springer, 3rd edition.

NASA (2006a), New Horizons, http://pluto.jhuapl.edu, Last accessed: April 21, 2006.

NASA (2006b), Solar System Exploration, http://solarsystem.nasa.gov/ missions/future1.cfm, Last accessed: January 5, 2007.

NASA/GSFC (2006), Lunar and Planetary Science, http:/nssdc.gsfc. nasa.gov/planetary, Last accessed: March 28, 2006.

NASA/JPL (2002), Deep Space 1, http://nmp.jpl .nasa.gov/ds1, Last accessed: April 10, 2006.

NASA/JPL (2005a), Galileo Legacy Site, http://galileo.jpl.nasa.gov, Last accessed: January 31, 2006.

NASA/JPL (2005b), Planetary Data System, http://pds.jpl.nasa.gov/ planets/choices/neptune1.htm, Last accessed: March 26, 2006.

NASA/JPL (2006a), Cassini-Huygens, http://saturn.jpl.nasa.gov, Last accessed: February 1, 2006.

NASA/JPL (2006b), Planetary Photojournal, http://photojournal.jpl. nasa.gov, Last accessed: January 19, 2006.

NASA/JPL (2006c), Solar System Dynamics, http://ssd.jpl.nasa.gov, Last accessed: July 3, 2006.

Noca, M.A., and R.W. Bailey (2004), Mission Trades for Aerocapture at Neptune, American Institute of Aeronautics and Astronautics, AIAA Paper 2004-3843, July 2000 . 
Noomen, R., Q.P. Chu, and A. Kamp (2003), Space Engineering and Technology III, Delft University of Technology, Faculty of Aerospace Engineering, Lecture notes ae3-803.

Ortega, G. (2005), Mission to Triton, PowerPoint Presentation, European Space Research and Technology Centre.

Perez, E. (2004), Ariane User's Manual, Arianespace, Issue 4, Revision 0.

Petropoulos, A.E., and J.M. Longuski (2000), Automated Design of Low-Thrust Gravity-Assist Trajectories, American Institute of Aeronautics and Astronautics, AIAA Paper 2000-4033, August 2000.

Petropoulos, A.E., and J.M. Longuski (2004), Shape-Based Algorithm for Automated Design of Low-Thrust, Gravity-Assist Trajectories, Journal of Spacecraft and Rockets, Vol. 41, No. 5, p. 787-796, Sep-Oct 2004.

Petropoulos, A.E., J.M. Longuski, and N.X. Vinh (1999), Shape-Based Analytic Representations of Low-Thrust Trajectories for Gravity-Assist Applications, American Astronautical Society, AAS Paper 99-337, August 1999.

Safipour, E. (2006), Trajectory Optimization around Neptune, University of Glasgow, UK.

Schlijper, R.M.H. (2002), The Gravity-Assist, Literature Report, Delft University of Technology, Faculty of Aerospace Engineering.

Schlijper, R.M.H. (2003), Genetic Algorithm Optimization of Planetary GravityAssist Missions to Pluto, Thesis Report, Delft University of Technology, Faculty of Aerospace Engineering.

Schouten, G. (2006), The Space Trajectory Analysis Tool and the Development of its Interplanetary Module, Thesis Report, Delft University of Technology, Faculty of Aerospace Engineering.

Sponnick, J.V., and M. Jensen (2004), Atlas Launch System Mission Planner's Guide, International Launch Services, Revision 10.

Starsem (2001), Soyuz User's Manual, Starsem, The Soyuz Company, Issue 3, Revision 0.

Wakker, K.F. (1997a), Astrodynamics I, Delft University of Technology, Faculty of Aerospace Engineering, Lecture notes ae4-873 (partly in Dutch).

Wakker, K.F. (1997b), Astrodynamics II, Delft University of Technology, Faculty of Aerospace Engineering, Lecture notes ae4-873 (partly in Dutch).

Wertz, J.R. (2001), Mission Geometry \& Orbit Constellation: Design and Management, El Segundo: Microcosm Press.

Zandbergen, B.T.C. (2004), Thermal Rocket Propulsion, Delft University of Technology, Faculty of Aerospace Engineering, Lecture notes ae4-S01. 



\section{Appendix A}

\section{Derivations Petropoulos}

- Derivation of equation (7.9):

$$
\dot{\theta}^{2}=\left(\frac{\mu}{r^{3}}\right) \frac{1}{\tan ^{2} \gamma+k_{12 s}+1}
$$

Divide equation (7.6) and (7.7) to get

$$
\frac{\ddot{r}-r \dot{\theta}^{2}+\frac{\mu}{r^{2}}}{2 \dot{r} \dot{\theta}+r \ddot{\theta}}=\tan \alpha
$$

Since the assumption is made that the thrust is (anti-)parallel to the velocity vector, it holds that $\tan \alpha=\tan \gamma$. Next, $\dot{r}$ and $\ddot{r}$ need to be expressed in the shape parameters and the derivatives of $\theta$, since these derivatives of $r$ do not occur in equation (7.9).

$$
\begin{aligned}
& \dot{r}=\frac{\mathrm{d} r}{\mathrm{~d} t}=\frac{\mathrm{d} r}{\mathrm{~d} \theta} \frac{\mathrm{d} \theta}{\mathrm{d} t}=k_{1} k_{2} \cos \left(k_{2} \theta+\phi\right) r \dot{\theta}=r \dot{\theta} \tan \gamma \\
& \ddot{r}=\frac{\mathrm{d} \dot{r}}{\mathrm{~d} t}=\frac{\mathrm{d} \dot{r}}{\mathrm{~d} \theta} \frac{\mathrm{d} \theta}{\mathrm{d} t}+\frac{\mathrm{d} \dot{r}}{\mathrm{~d} \dot{\theta}} \frac{\mathrm{d} \dot{\theta}}{\mathrm{d} t}+\frac{\mathrm{d} \dot{r}}{\mathrm{~d} r} \frac{\mathrm{d} r}{\mathrm{~d} t}=-k_{12 s} r \dot{\theta}^{2}+r \ddot{\theta} \tan \gamma+r \dot{\theta}^{2} \tan ^{2} \gamma
\end{aligned}
$$

Now substitute equations (A.2) and (A.3) in equation (A.1) and divide both numerator and denominator of the right-hand side by $r$ to get

$$
\tan \gamma=\frac{-k_{12 s} \dot{\theta}^{2}+\ddot{\theta} \tan \gamma+\dot{\theta}^{2} \tan ^{2} \gamma-\dot{\theta}^{2}+\frac{\mu}{r^{3}}}{2 \dot{\theta}^{2} \tan \gamma+\ddot{\theta}}
$$

Now bring the denominator of the right-hand side to the left-hand side to obtain

$$
2 \dot{\theta}^{2} \tan ^{2} \gamma+\ddot{\theta} \tan \gamma=-k_{12 s} \dot{\theta}^{2}+\ddot{\theta} \tan \gamma+\dot{\theta}^{2} \tan ^{2} \gamma-\dot{\theta}^{2}+\frac{\mu}{r^{3}}
$$

The term $\ddot{\theta} \tan \gamma$ cancels out. Rearranging gives

$$
\dot{\theta}^{2}\left(\tan ^{2} \gamma+k_{12 s}+1\right)=\frac{\mu}{r^{3}}
$$

This is obviously equal to equation (7.9), and thus this equation has been derived. 
- Derivation of equation (7.10):

$$
a_{T}=\left(\frac{\mu}{r^{2}}\right) \frac{\tan \gamma}{2 \cos \gamma}\left[\frac{1}{\tan ^{2} \gamma+k_{12 s}+1}-\frac{k_{2}^{2}\left(1-2 k_{1} s\right)}{\left(\tan ^{2} \gamma+k_{12 s}+1\right)^{2}}\right]
$$

Add equations 7.6 and 7.7 to get

$$
\ddot{r}-r \dot{\theta}^{2}+\frac{\mu}{r^{2}}+2 \dot{r} \dot{\theta}+r \ddot{\theta}=F(\sin \gamma+\cos \gamma)
$$

Now substitute the relations for $\dot{r}$ and $\ddot{r}$ (equations (A.2) and (A.3) respectively) and rearrange:

$$
\begin{aligned}
a_{T}(\sin \gamma+\cos \gamma)= & -k_{12 s} r \dot{\theta}^{2}+r \ddot{\theta} \tan \gamma+r \dot{\theta}^{2} \tan ^{2} \gamma-r \dot{\theta}^{2}+\frac{\mu}{r^{2}}+2 r \dot{\theta}^{2} \tan \gamma+r \ddot{\theta} \\
= & r\left((1+\tan \gamma) \ddot{\theta}+\left(\tan ^{2} \gamma+2 \tan \gamma-k_{12 s}-1\right) \dot{\theta}^{2}+\frac{\mu}{r^{3}}\right) \\
= & r((1+\tan \gamma) \ddot{\theta}+(2 \tan \gamma(1+\tan \gamma)- \\
& \left.\left.\quad\left(\tan ^{2} \gamma+k_{12 s}+1\right)\right) \dot{\theta}^{2}+\frac{\mu}{r^{3}}\right) \\
= & r\left((1+\tan \gamma) \ddot{\theta}+2 \tan \gamma(1+\tan \gamma) \dot{\theta}^{2}-\frac{\mu}{r^{3}}+\frac{\mu}{r^{3}}\right)
\end{aligned}
$$

The last step uses equation (A.6). Both sides can be divided by $\cos \gamma$ to result in

$$
a_{T}=\frac{r}{\cos \gamma}\left(\ddot{\theta}+2 \dot{\theta}^{2} \tan \gamma\right)
$$

Now an expression needs to be find for $\ddot{\theta}$. This is done by taking the derivative of equation (A.6). For convenience $c \equiv \cos \left(k_{2} \theta+\phi\right)$.

$$
\begin{aligned}
2 \dot{\theta} \ddot{\theta}\left(\tan ^{2} \gamma+k_{12 s}+1\right)+\dot{\theta}^{2} \frac{\mathrm{d}}{\mathrm{d} t}\left(k_{1}^{2} k_{2}^{2} c^{2}+k_{1} k_{2}^{2} s+1\right) & =-\frac{3 \mu \dot{r}}{r^{4}} \\
2 \ddot{\theta} \ddot{\theta}\left(\tan ^{2} \gamma+k_{12 s}+1\right)+\dot{\theta}^{3}\left(-2 k_{1}^{2} k_{2}^{3} s c+k_{1} k_{2}^{3} c\right) & =-\frac{3 \mu \dot{\theta} \tan \gamma}{r^{3}} \\
2 \ddot{\theta}\left(\tan ^{2} \gamma+k_{12 s}+1\right)+\dot{\theta}^{2} k_{2}^{2} \tan \gamma\left(1-2 k_{1} s\right) & =-\frac{3 \mu \tan \gamma}{r^{3}}
\end{aligned}
$$

Now isolating $\ddot{\theta}$ gives

$$
\ddot{\theta}=\frac{-\mu \tan \gamma}{2 r^{3}\left(\tan ^{2} \gamma+k_{12 s}+1\right)}\left(3+\frac{k_{2}^{2}\left(1-2 k_{1} s\right)}{\tan ^{2} \gamma+k_{12 s}+1}\right)
$$

Substituting this relation into equation (A.9) gives

$$
a_{T}=\frac{r \mu \tan \gamma}{r^{3} \cos \gamma}\left[\frac{2-3 / 2}{\tan ^{2} \gamma+k_{12 s}+1}-\frac{k_{2}^{2}\left(1-2 k_{1} s\right)}{2\left(\tan ^{2} \gamma+k_{12 s}+1\right)^{2}}\right]
$$

Rearranging gives

$$
a_{T}=\left(\frac{\mu}{r^{2}}\right) \frac{\tan \gamma}{2 \cos \gamma}\left[\frac{1}{\tan ^{2} \gamma+k_{1} k_{2}^{2} s+1}-\frac{k_{2}^{2}\left(1-2 k_{1} s\right)}{\left(\tan ^{2} \gamma+k_{1} k_{2}^{2} s+1\right)^{2}}\right]
$$

and thus equation (7.10) has been derived. 